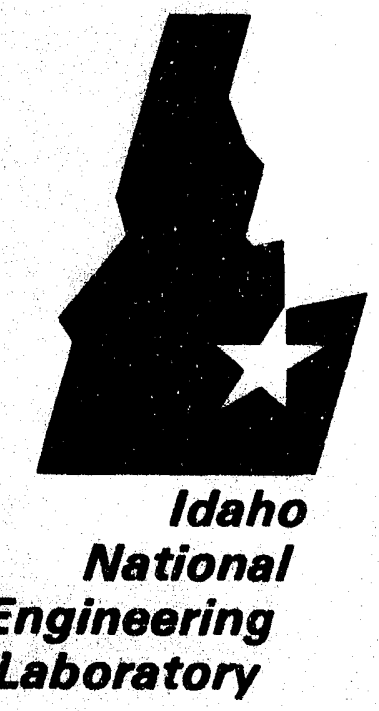

Managed

by the U.S.

Department

of Energy
EGG-MS-10992

September 1993

\section{DESIGN AND CONSTRUCTION OF AN \\ IMAGING INSTRUMENT FOR STUDYING \\ ION EMISSION FROM PURE ION EMITTERS}

John E. Olson

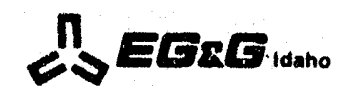

Work performed under DOE Contract No. DE-AC07-761D01570 


\section{DISCLAIMER}

This report was prepared as an account of work sponsored by an agency of the United States Thernment. Neither the United States Governility or responsiemployees, makes any warranty, express or implicd, or assues bility for the accuracy, completeness, or usefulness of any inform process disclosed, or represents that its use would not infringe privately owned re trademark process disclosed, or represents that is use would nocess, or service by trade name, trademark. ence herein to any specific commercial product, process, or ser imply its endorsement, recommanufacturer, or otherwise does not necessarily constute or imply its ency thereof. The views mendation, or favoring by the United States Government or any agency thect those of the authors expressed herein do not

and opinions of authors expressed herein do not
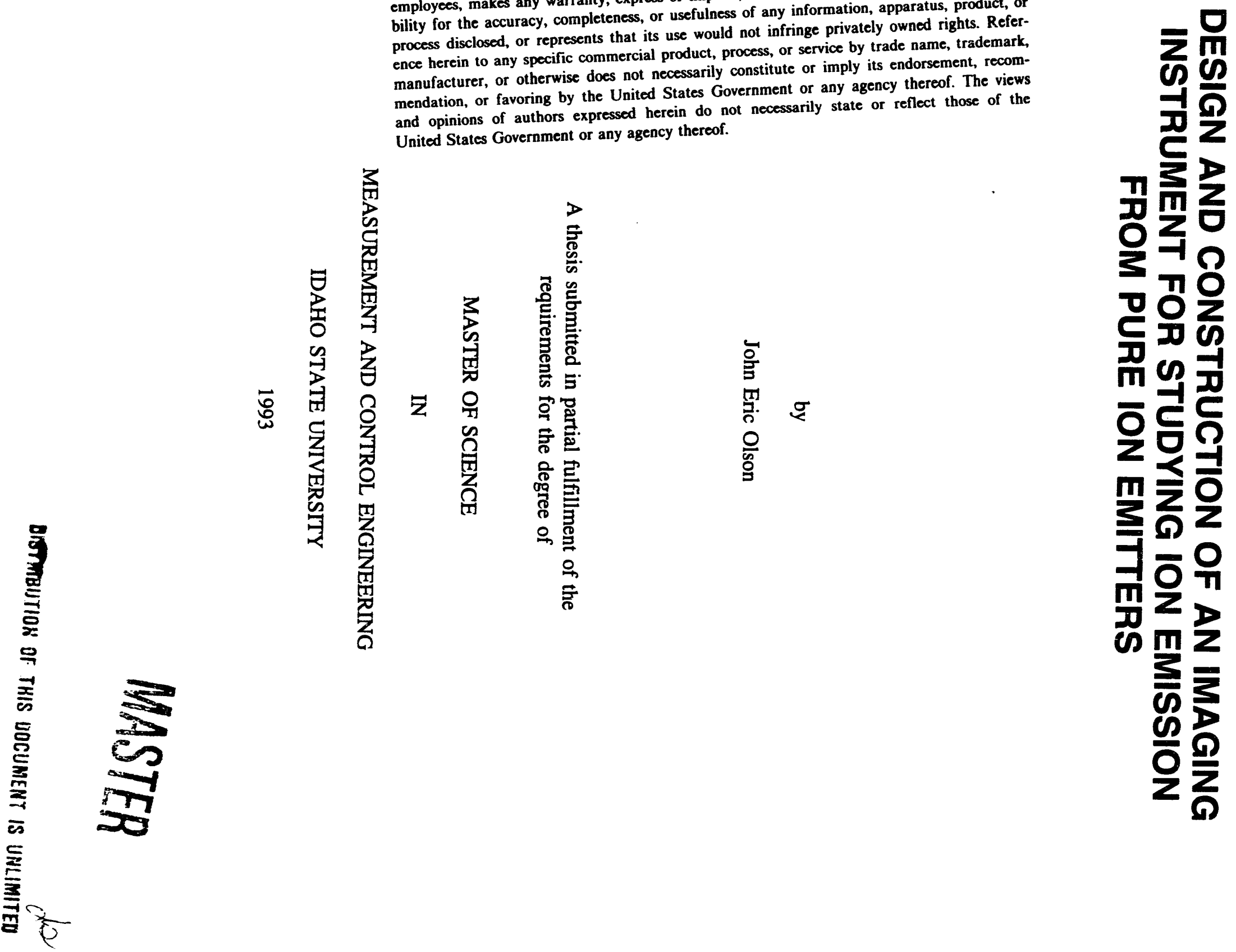


\title{
DESIGN AND CONSTRUCTION OF AN IMAGING INSTRUMENT FOR STUDYING ION EMISSION FROM PURE ION EMITTERS
}

\author{
John Eric Olson \\ Thesis Abstract - Idaho State University (1993)
}

The development of new ion sources is important in the area of surface analysis to make it easier to perform more sensitive and accurate analyses. In surface analysis a primary ion beam composed of a single species can help when predicting and interpreting the results. Therefore, much interest and effort has been focused on producing pure ion emitters.

An instrument has been designed and constructed to view the current densities of the ions being emitted from pure ion emitters. The instrument electrostatically accelerates and focuses the ion beam onto a microchannel plate detector equipped with a phosphor screen for viewing the images. These images are used to identify areas of enhanced ion emission. Once these areas are identified, the investigator can use other instruments to analyze them, and hopefully develop a better understanding of the chemistry and physics involved in the ion emission process. A computer based control system has been integrated into the system to simplify the operation of the instrument and provide safety features to protect the hardware from damage. A closed-circuit video camera system is used to allow the images to be remotely viewed during imaging procedures.

Experiments show that the instrument has a lower detection limit of $7.45 \times 10^{3}$ ions $/ \mathrm{sec} / \mathrm{mm}^{2}$ and a spatial resolution of approximately $3-4 \mu \mathrm{m}$. Results from imaging cesium zeolite and perrhenate ion sources indicate that the ions are primarily being emitted from the surface of the sources and not from the interfacial region between the substrate and the emitter material. 


\section{ACKNOWLEDGMENTS}

I would like to thank Sondra Olson and Associated Western Universities Inc. for giving me the opportunity to do the research at the INEL necessary to earn my degree. A special thanks needs to be extended to Dr. Jim Delmore for making available to me all of the resources, support, and advice necessary to complete my work on the imaging instrument. Along with Jim, Tony Appelhans and Dave Dahl have contributed significantly with my work. Two people that need mentioning are the dean of the college of Engineering, Hary Charyulu, and his wife Cindy. They are good friends that have made going to school at ISU a pleasurable experience. Of course my girl friend, Jodi, has been very supportive while I have been in school. The last people that I would like to mention are my folks, Robert and Kathleen Olson. They have been very helpful and have offered much support for me while completing my degree. This work has been supported by the Division of Chemical Sciences, Basic Energy Sciences, Office of Energy Research, Department of Energy under contract 3ED102.y

John E. Olson 


\section{TABLE OF CONTENTS}

ABSTRACT $\ldots \ldots \ldots \ldots \ldots \ldots \ldots \ldots \ldots \ldots \ldots \ldots \ldots \ldots \ldots \ldots \ldots \ldots$ ii

LIST OF FIGURES $\ldots \ldots \ldots \ldots \ldots \ldots \ldots \ldots \ldots \ldots \ldots \ldots \ldots \ldots$ vii

INTRODUCTION $\ldots \ldots \ldots \ldots \ldots \ldots \ldots \ldots \ldots \ldots \ldots \ldots \ldots \ldots \ldots \ldots \ldots$

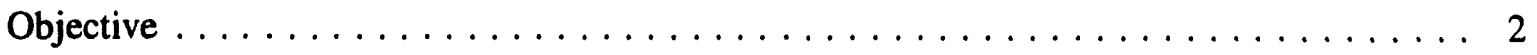

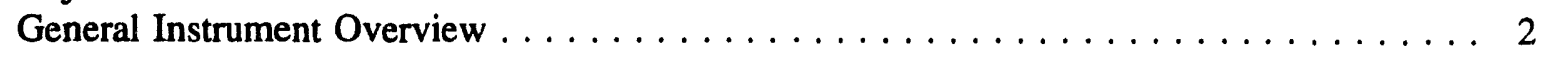

IMAGING TECHNIQUES $\ldots \ldots \ldots \ldots \ldots \ldots \ldots \ldots \ldots \ldots \ldots \ldots \ldots$

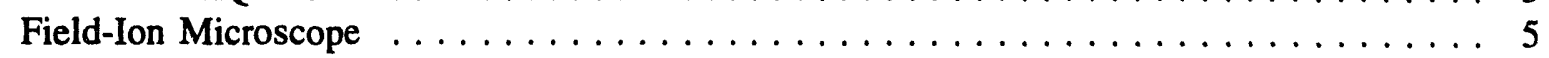

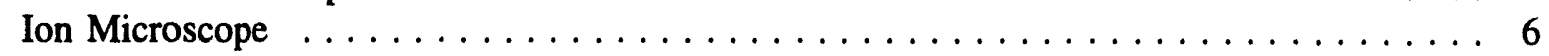

Scanning Ion Microprobe $\ldots \ldots \ldots \ldots \ldots \ldots \ldots \ldots \ldots \ldots \ldots \ldots$

Direct-Imaging Scanning Ion Microprobe $\ldots \ldots \ldots \ldots \ldots \ldots \ldots \ldots \ldots \ldots$

Imaging Instrument (This Paper) $\ldots \ldots \ldots \ldots \ldots \ldots \ldots \ldots \ldots \ldots \ldots$

INSTRUMENT DESIGN AND CONSTRUCTION $\ldots \ldots \ldots \ldots \ldots \ldots \ldots \ldots \ldots \ldots \ldots$

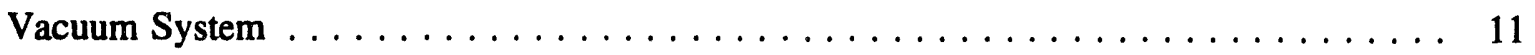

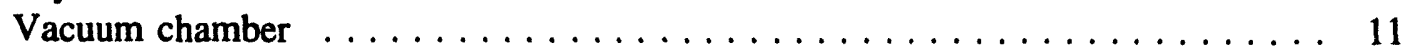

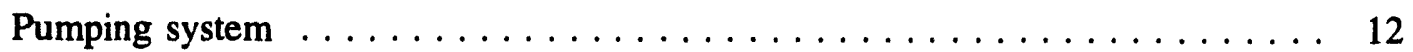

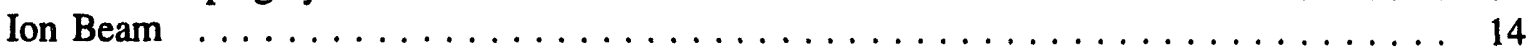

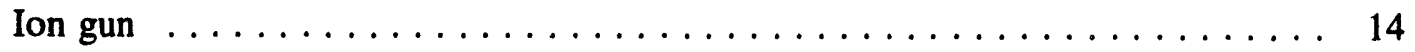

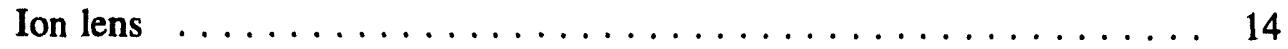

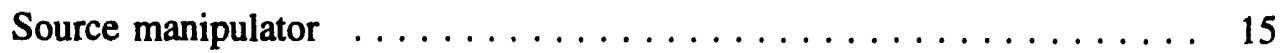

Ion source assembly $\ldots \ldots \ldots \ldots \ldots \ldots$

Ion gun electronics $\ldots \ldots \ldots \ldots \ldots \ldots \ldots \ldots \ldots \ldots \ldots$

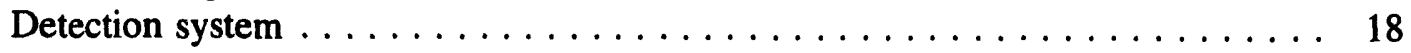

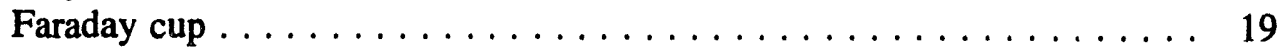

Microchannel plate . . . . . . . . . . . . . . . . 20

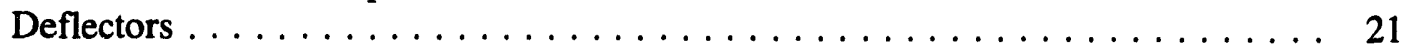

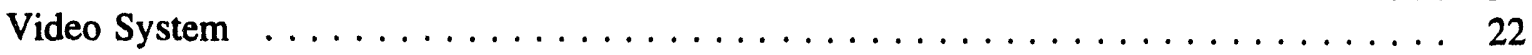

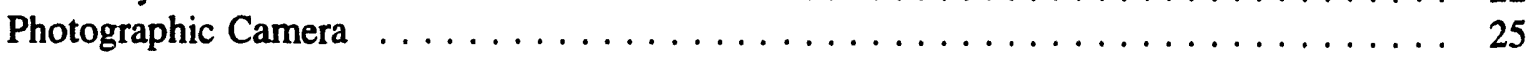

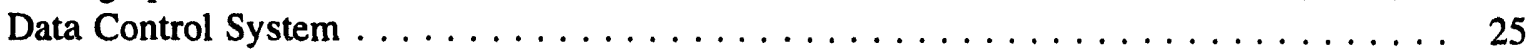

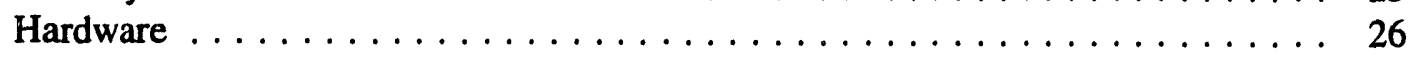

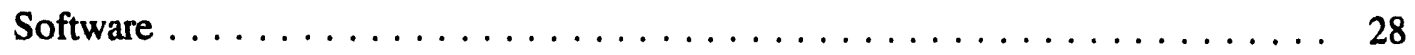

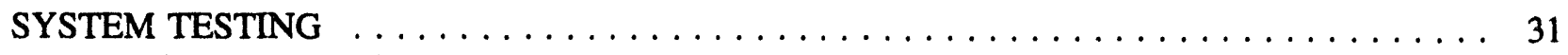

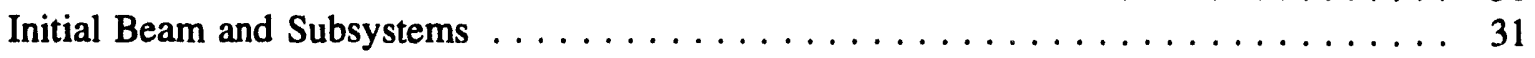

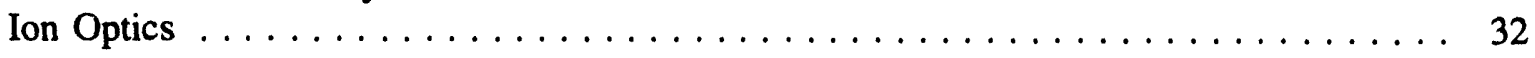

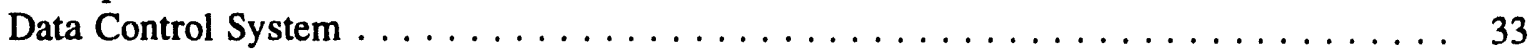

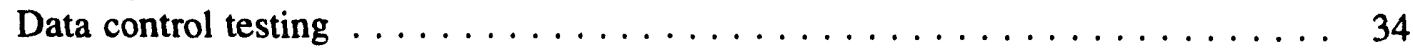

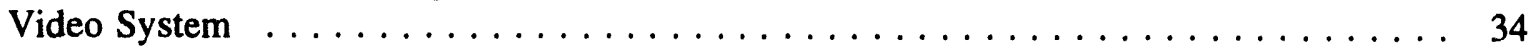

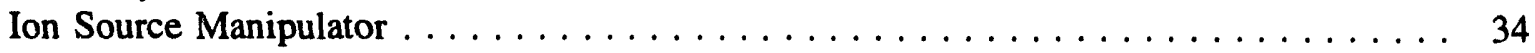


EXPERIMENTAL RESULTS $\ldots \ldots \ldots \ldots \ldots \ldots \ldots \ldots \ldots \ldots \ldots \ldots \ldots \ldots \ldots$

Minimum Detection Limit $\ldots \ldots \ldots \ldots \ldots \ldots \ldots \ldots \ldots \ldots \ldots \ldots \ldots \ldots$

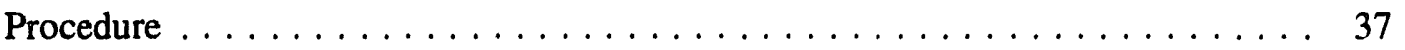

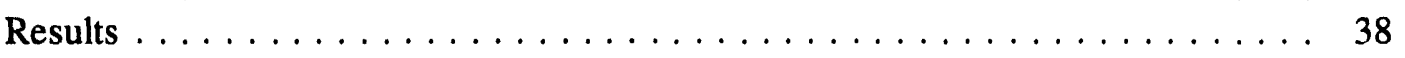

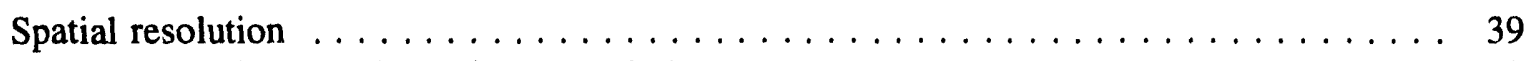

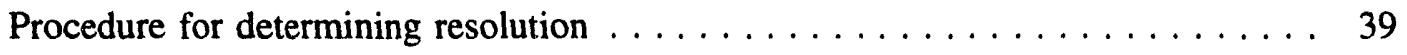

Results . . . . . . . . . . . . . . . . . . . . . . . . . 39

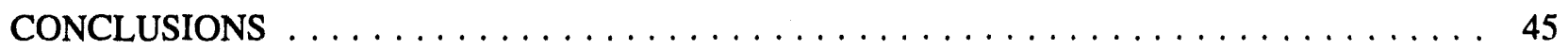

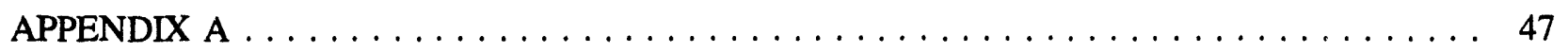

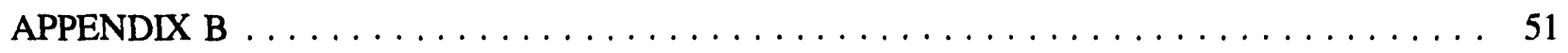

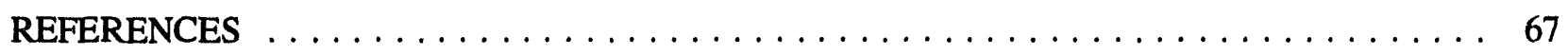




\section{LIST OF FIGURES}

Figure $1: \quad$ Schematic of imaging instrument $\ldots \ldots \ldots \ldots \ldots \ldots \ldots$

Figure $2: \quad$ Direct imaging ion microscope $\ldots \ldots \ldots \ldots \ldots \ldots \ldots \ldots \ldots$

Figure 3: $\quad$ Scanning ion microprobe $\ldots \ldots \ldots \ldots \ldots \ldots \ldots \ldots \ldots \ldots \ldots$

Figure 4: $\quad$ Direct-imaging scanning ion microprobe $\ldots \ldots \ldots \ldots \ldots \ldots$

Figure 5: $\quad$ Isometric view of vacuum chamber $\ldots \ldots \ldots \ldots \ldots \ldots$

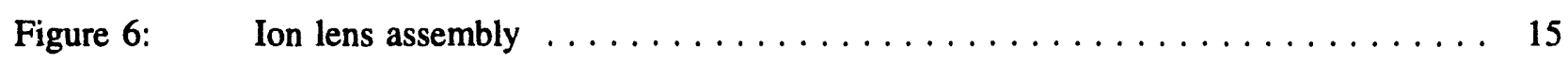

Figure $7: \quad$ Ion source mounting assembly $\ldots \ldots \ldots \ldots \ldots \ldots \ldots$

Figure 8: $\quad$ Tube source assembly $\ldots \ldots \ldots \ldots \ldots \ldots \ldots \ldots \ldots \ldots$

Figure 9: $\quad$ Filament supply schematic $\ldots \ldots \ldots \ldots \ldots \ldots \ldots \ldots$

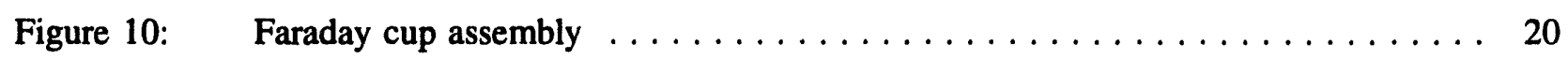

Figure 11: $\quad$ Microchannel plate schematic $\ldots \ldots \ldots \ldots \ldots \ldots \ldots \ldots \ldots \ldots \ldots$

Figure 12: $\quad$ Deflector assembly $\ldots \ldots \ldots \ldots \ldots \ldots \ldots \ldots \ldots \ldots \ldots \ldots \ldots$

Figure 13: $\quad$ Top view of camera arrangement $\ldots \ldots \ldots \ldots \ldots \ldots \ldots \ldots$

Figure 14: $\quad$ Schematic of signal conditioning circuit $\ldots \ldots \ldots \ldots \ldots \ldots \ldots$

Figure 15: $\quad$ Main interface screen $\ldots \ldots \ldots \ldots \ldots \ldots \ldots \ldots \ldots \ldots \ldots$

Figure 16: Cesium zeolite source: a) light micrograph $64 \mathrm{x}$ b) ion micrograph $110 \mathrm{x}$ c) ion

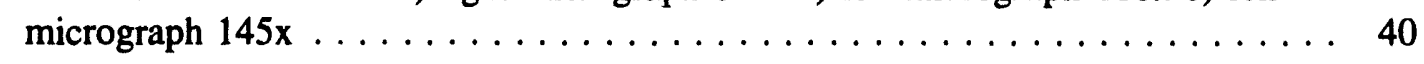

Figure 17: Perrhenate ion source a) light micrograph $64 x$ b) ion micrograph $160 x \ldots 42$

Figure 18: Perrhenate ion source \#2 a) light micrograph $64 x$ b) ion micrograph $48 x \mathrm{c}$ ) ion

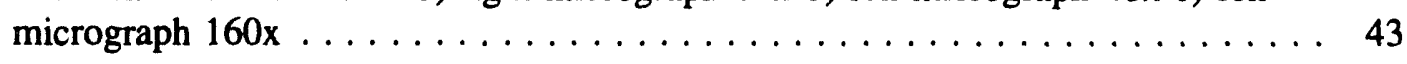

Figure 19: $\quad$ Schematic of signal conditioning circuit $\ldots \ldots \ldots \ldots \ldots \ldots$

Figure 20: $\quad$ Control circuitry for high voltage supply which floats the filament supply . . . . 49

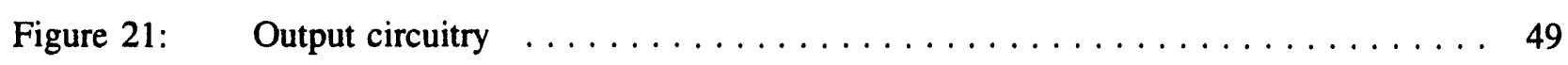


Figure 22: $\quad$ Interlock latching circuitry $\ldots \ldots \ldots \ldots \ldots \ldots \ldots \ldots \ldots \ldots \ldots \ldots \ldots$

Figure 23: $\quad$ Main interface screen $\ldots \ldots \ldots \ldots \ldots \ldots \ldots \ldots \ldots \ldots \ldots$

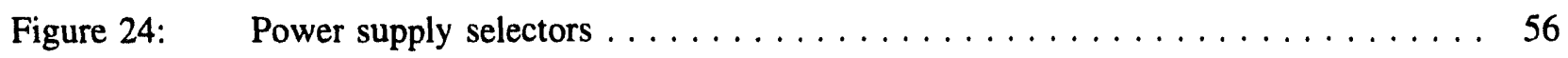

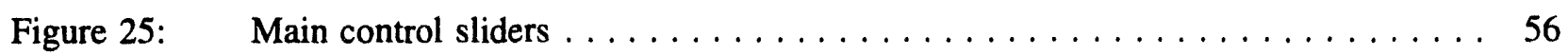

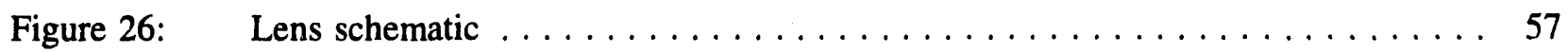

Figure 27: $\quad$ File management window $\ldots \ldots \ldots \ldots \ldots \ldots \ldots \ldots \ldots \ldots$

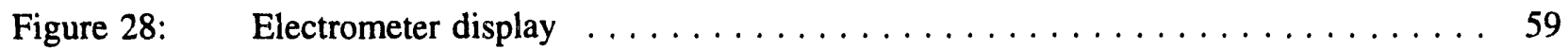

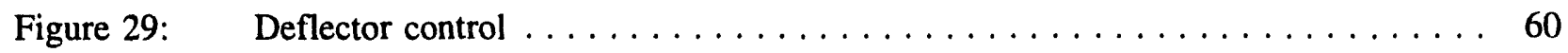

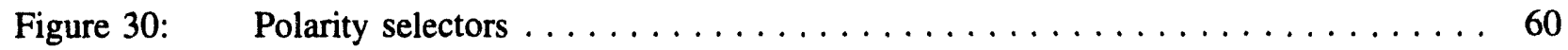

Figure 31: $\quad$ Parts layout of signal conditioning circuit $\ldots \ldots \ldots \ldots$

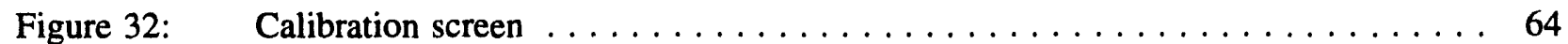




\section{CHAPTER I INTRODUCTION}

Many advancements in science and technology have come about as the result of ion bombardment processes such as those used in surface analysis. Surface analysis techniques are used to ascertain the chemical composition of samples ranging from metals to biological tissues. In these processes it is important to have an ion beam of a single species to help in predicting and interpreting the results and minimize contaminants from other ion species. Therefore, much interest and effort has been focused on producing pure ion emitters. There are instruments available to determine the species of ions being emitted from these emitters, but most do not give a spatial image of the current densities of the ions being emitted from the emitter surface. Delmore et al.' have already demonstrated the usefulness of visual observations of the ion emission process. For example, by observing where the ions were being formed in an $\mathrm{SF}_{6}$ autoneutralizing ion gun, they determined that a spiral- or cylindrical-shaped filament could produce ions more efficiently than straight filaments. These results illustrate the value of a dedicated instrument for observing the ion emission processes as a tool for evaluating, explaining, and hopefully leading to more and better ion sources in the future.

This thesis describes the design and construction of an instrument for viewing the ion current densities present in the emission process of pure ion emitters. The primary use for this instrument will be to study low energy ion sources (up to $10 \mathrm{KeV}$ ) that are used in surface analysis. The understanding and development of new ion sources for use in surface analysis is necessary to (i) increase the sensitivity of the analysis; and (ii) overcome some of the problems, such as surface charging, that are inherent in ion bombardment techniques. A better understanding of the processes involved in the emission of ions from their sources is essential to the continued progress in all areas of ion beam technology. This knowledge may lead to the production of more efficient ion emitters, new methods of producing ions, ion sources better suited for the particular application, and mors efficient ion optics for delivering the particles to their end use.

In the area of surface analysis, ions have been used for many years to bombard samples to produce secondary ions that are emitted from the surface of the sample. The mass of the secondary ions is then analyzed to determine the chemical composition of the sample. This process is known as secondary ion mass spectrometry (SIMS). SIMS analysis can be used to analyze the first monolayer of a sample (static SIMS), or a depth profile of the composition of the sample can be obtained in dynamic SIMS.

In SIMS analysis, the more secondary ions emitted from the surface of the sample the easier it becomes to perform a more accurate and sensitive analysis on the sample. Secondary ion yield depends on many factors including the type of primary ion, the energy of the primary beam, and the angle of incidence on the sample. Therefore, the primary beam plays an important role in the overall sensitivity in SIMS analysis. Bombardment of the sample with a primary beam composed of reactive gas ions such as Cesium or Oxygen ions can be used to enhance the production of secondary metal ions from the surface of the sample. Heavy ions or molecular ions may also augment secondary ion emission. The instrument described in this thesis can help in developing new ion sources that increase the secondary ion yield, and thus increase the sensitivity of the analysis.

A problem that arises in SIMS analysis is that while examining insulating materials a charge can build up on the surface of the sample, thus degrading the signal. This charge is the result of an imbalance of charge imparted to the sample by the primary beam and the charged particles that are emitted from the sample by the sputtering process. This charge build up is most prevalent with a 
positive primary ion beam and can be reduced by using either an anionic or neutral species for the primary beam. When using a neutral or anionic beam, the charge build up on the sample can further be controlled by alternating between extracting negative and positive ions, and regulating the frequency and duty cycle of extraction (pulsed extraction). ${ }^{2}$ Thus the development of pure anion emitters can be another beneficial use of the instrument.

\section{Objective}

The objective of this thesis project is to construct an instrument for direct imaging of the primary ions emitted from ion sources to gather qualitative data pertaining to the spatial origin of the areas of ion emission. This is unlike the ion microscope, which images secondary ions in an attempt to determine compositions of samples. The imaging instrument will image the ions being emitted from the primary ion sources themselves in an attempt to determine some of the processes involved in ion emission. The instrument is versatile and able to adapt to various designs of ion sources. It is also capable of imaging both anions and cations. Based on preliminary results, it is expected that this will be a useful analytical tool, and, when used in conjunction with other analysis techniques, such as scanning electron microscopy (SEM), a better understanding of these processes can be developed.

The thesis is split into several chapters that will discuss: 1) some of the imaging techniques that are used in other ion imaging instruments, as well as the technique used for the instrument developed in this project; 2) the overall design and construction of the instrument; 3) the testing of the instrument and any modifications that were necessary to ensure proper performance of the instrument; 4) the experimental results, including the procedures used to determine the lower detection limit and spatial resolution of the instrument, and a contrast of ion micrographs with light micrographs of the same sources, thus demonstrating the operability and usefulness of the instrument; and 5) any conclusions that could be drawn from the preliminary experiments with the instrument. Appendix A describes the theory of operation of the signal conditioning circuit. A complete user's manual on the operation and calibration of the imaging instrument can be found in Appendix B.

It is not the intent of this thesis to explain the phenomenon associated with ion emission, but to construct and demonstrate the operability of an instrument to help in the study of such processes. The instrument will be used in future experiments and the results along with other analysis will be used to help explain the process of how ion emission occurs from solid state ion emitters.

\section{General Instrument Overview}

The general functionality of the instrument was known in advance. However, many subassemblies and systems had to be designed and integrated into the overall instrument to create a usable analytical tool for the investigation of the ion emission processes. A schematic view of the imaging instrument is shown in Figure 1. The schematic shows most of the major components with the exception of the power supplies, computer control, and ion pump. The complete instrument had to be designed and constructed, starting with the source chamber. This included purchasing standard components, as well as custom designing some of the assemblies to finish the construction of the system. The entire design will be presented in detail in the system design portion of this thesis.

Since the goal of this project was to study pure ion emitters, there was no need to have any mass resolving power built into the instrument. This simplified the overall design and enhanced the 


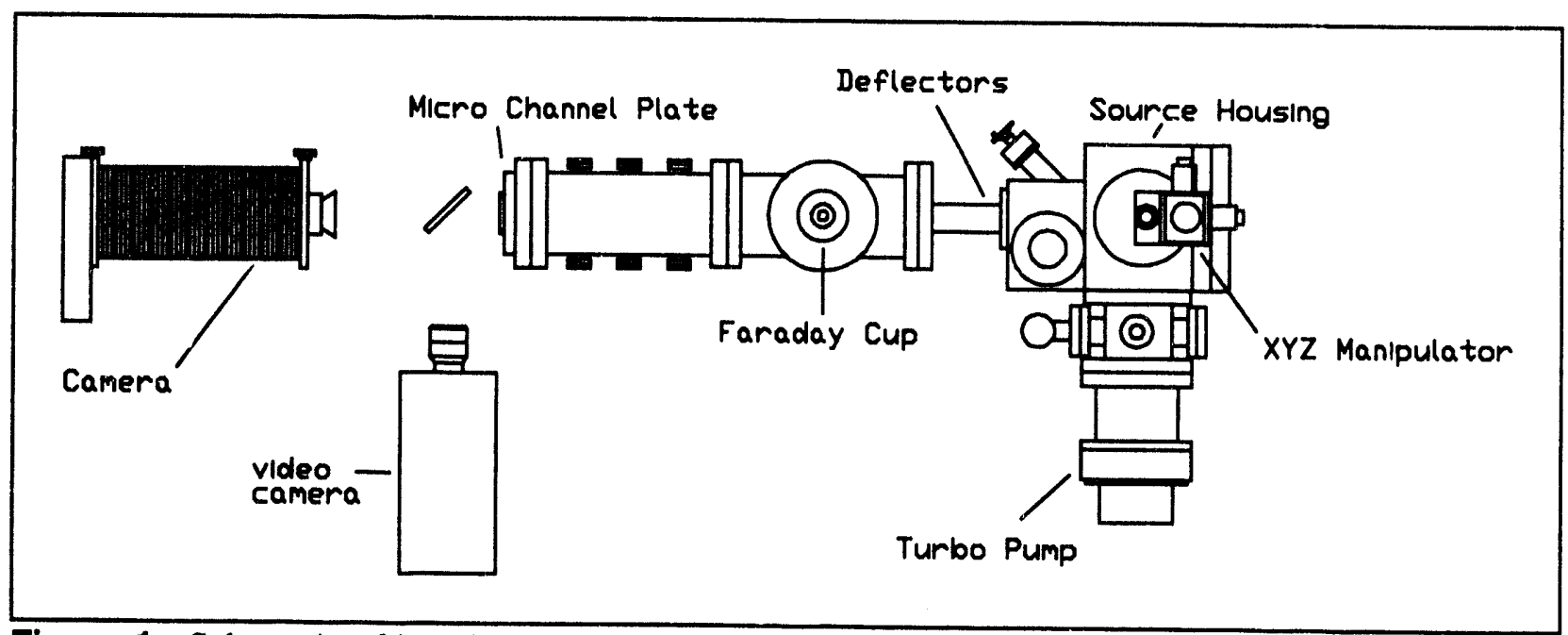

Figure 1: Schematic of imaging instrument

sensitivity of the instrument by reducing the complexity of the ion optics in the system. The ion gun is cylindrically symmetric and constructed using components from the National Bureau of Standards (NBS). The design allows for easy removal and modification of the ion optics to permit the imaging qualities to be changed as might be necessary with various ion sources. The ion source is floated at high voltage between 0 and $\pm 10 \mathrm{kV}$ and is mounted on an $\mathrm{XYZ}$ manipulator. The manipulator allows for precise alignment of small areas of the source with the center line of the ion optics. This permits high resolution imaging of all areas of an ion emitter. The primary ion beam is accelerated and focused onto a microchannel plate (MCP) with a phosphor screen for direct imaging. Images of the ion source are recorded using a photographic camera equipped with either polaroid film for immediate use or negative film for further development.

A unique personal computer-based data control system was developed to make the instrument easier to operate while also providing certain safety features to protect the hardware. The control system consisted of the computer, along with a signal conditioning circuit. The signal conditioning circuit provided zero and span correction for the control signals from the computer. It also used feedback to derive the control signals for some of the high voltage power supplies. The feedback was used to make some of the high voltage supplies track the voltage adjustments made to the ion source. This reduced the risk of arcing in the ion lens assembly. The software for the computer interface was developed using a graphical user interface that required writing a substantial amount of code in the $\mathrm{C}$ language. Along with programming in $\mathrm{C}$, device drivers were developed in assembler to speed up the response of the digital-to-analog converter (DAC) board. An IEEE-488 interface was used to remotely control the electrometer that was used to measure the ion current.

A video system was integrated into the instrument to help in the observation of the images while both focusing the instrument and positioning the ion source. Future plans include incorporating a time lapse video recorder for archiving ion sources in which the ion emission is changing with time. Also, the video system could be used to expedite the evolution of the instrument into the image processing arena through the use of a frame grabber, thus allowing more sophisticated techniques to be implemented in evaluating the data obtained through the imaging of these sources. 
The experimental portion of the thesis shows that the instrument has a lower detection limit on the order of $7.45 \times 10^{3}$ ions $/ \mathrm{sec} / \mathrm{mm}^{2}$ and a spatial resolution on the order of $3-4 \mu \mathrm{m}$. The preliminary results obtained while imaging both the cesium and perrhenate ion emitters clearly show that a large number of the ions being emitted from these sources are coming from the surface of the material and not from the interfacial region between the substrate and the material. All indications lead us to believe that the imaging instrument will be a useful tool for investigating the phenomenon associated with the emission of ions from a wide variety of ion emitters. 


\section{CHAPTER II \\ IMAGING TECHNIQUES}

As discussed in Chapter 1, the type of charged particles used in the primary beam in surface analysis play an important role in determining the overall sensitivity of the analysis and can be used to overcome some of the problems, such as surface charge build up, involved in ion bombardment. By developing a better understanding of how the ions are emitted from sources, more ion sources may result that can benefit ion beam technology. There are many analytical tools available for the study of emission characteristics of ion sources. However, most instruments that are available do not allow the visual observation of the current densities of the ions being emitted, at least from the primary source. An imaging instrument designed to study the emission of ions from the surfaces of the primary ion emitters could give an insight into where and how the ions are formed that is not available from other instruments.

The first ion imaging instrument was Müller's field ion microscope, which was introduced in 1951. Throughout the years ion imaging has evolved and several types of imaging instruments have been developed in the area of secondary ion mass spectrometry (SIMS) analysis. Imaging SIMS instruments include direct imaging (ion microscope), scanning ion microprobes, or direct-imaging scanning ion microprobes. Since this thesis describes the construction of an imaging instrument, this chapter will discuss the principles behind these various imaging instruments and some of the techniques used to improve their imaging qualities and sensitivities. The last portion of the chapter will discuss the imaging technique used in the imaging instrument of this project.

\section{Field-Ion Microscope}

The first ion imaging instrument, introduced in 1951 by Erwin Müller, is the field-ion microscope(FIM). Field ionization is used to produce ions and images with resolutions on the atomic scale can be produced. In the FIM, a small amount of pure inert gas such as helium is introduced into a vacuum chamber and a finely polished refractory metal or alloy tip, 100 to $3000 \AA$ in diameter ${ }^{3}$, is held at a positive potential. Due to polarizing effects the gas molecules are attracted toward the metal tip. The high potential fields present near the tip of the metal sample cause the gas molecules to be ionized near the surface. The ions are then radially accelerated from the tip toward a phosphor screen where the scintillations can be observed. Furthermore, the ionization probability is higher directly above the individual atoms protruding from the metal sample 4 . Thus bright spots in the image correspond to the location of individual atoms on the surface of the specimen. By observing the geometric patterns formed in the images the crystalline structure of the surface of the metal sample can be determined. By carefully controlling the field evaporation of atoms from the surface, successive field-ion micrographs can reveal 3 dimensional qualities of the atomic structure of the sample.

A problem with the early versions of the FIM was the relatively small current densities and resulting image intensities of the ion micrographs. The small ion currents forced the use of gases that had a good phosphor efficiency. Unfortunately, helium, the preferred choice, has a relatively high ionization potential and the resulting fields produced near the tip of the source could only be tolerated by the much stronger refractory metals. One method to overcome this problem was to use a differentially pumped system in which the ionization gas pressure could be increased near the tip of the sample while maintaining the flight tube ai a much lower pressure ${ }^{3}$. This allowed higher ion 
currents while maintaining the resolution and also permitted the use of lower fields to enable the examination of different metals. Other solutions involved image conversion from ions to electrons and external image intensifiers. With the introduction of the channel plate detector and subsequent use in FIM demonstrated by Turner et al..$^{5}$, lower ion currents could be tolerated and other ionizing gases could be used to form the FIM images.

One major disadvantage to the original FIM is the fact that the images do not disclose any information about the atomic species forming the FIM patterns. In 1968 Müller et al. ${ }^{6}$ described an instrument that attempted to overcome this problem, the atom-probe field-ion microscope(AP FIM). The AP FIM combined the conventional FIM with a time of flight mass spectrometer to identify the atomic species on the surface creating the FIM patterns. Short duration high voltage pulses were used to field evaporate atoms from the surface of the sample. The resulting ions passed through an aperture in the phosphor screen and into a mass spectrometer for identification. The sample was mounted on a manipulator allowing any area of the FIM image to be positioned over the aperture in the phosphor screen and successively mass analyzed.

In 1974 Panitz described an imaging version of the AP FIM which allowed the chemical composition of a large area of the surface to be mass analyzed while maintaining the resol"'ion of the original FIM. The instrument is a time of flight mass analyzer in which the metal ions are field evaporated from the sample by a short duration high voltage pulse. After a delay, which is variable depending on what ion species is to be imaged, the channel plate is gated on to allow the imaging of the ions. Panitz used a dual (chevron) channel plate while Waugh used a single channel plate to image the ions ${ }^{7}$.

\section{Ion Microscope}

In 1962 Castaing and Slodzian described the first secondary ion mass spectrometer that gave a spatial resolution of $1 \mu \mathrm{m}$. In this instrument the image was viewed on a fluorescent screen ${ }^{8}$. Since a mass resolved image of the sample is available for immediate viewing this instrument is considered a direct imaging instrument. Further improvements were made to the ion microscope and a commercial version was marketed by CAMECA S. A. as the IMS 300 ion microscope.

In an ion microscope a sample is bombarded by a large diameter primary beam. Primary beams are typically in the range of $25-400 \mu \mathrm{m}$ in diameter. Argon $\left(\mathrm{Ar}^{+}\right)$is a common primary ion source but other ions can be used to enhance the emission of electro-negative or -positive secondary ions. Sputtered secondary ions from the sample are focused into a mass and energy filter. The mass resolved ions are subsequently focused onto a scintillator screen where an image of the current density of the particular secondary ion species present on the surface of the sample can be viewed. A schematic view of the arrangement in a direct imaging instrument can be seen in Figure 2.

The ion microscope provides qualitative data pertaining to the ion current and no quantitative data can be directly measured. Furman and Morrison ${ }^{9}$ have described a technique to infer quantitative data from the images by digitizing the pixel information with high speed image processing equipment and along with special calibration and interpretation procedures the intensities of the pixels correspond to specific ion counts. Odem et al. ${ }^{10}$ have used another technique for obtaining quantitative information using the ion microscope by using a resistive anode encoder (RAE) in conjunction with a 


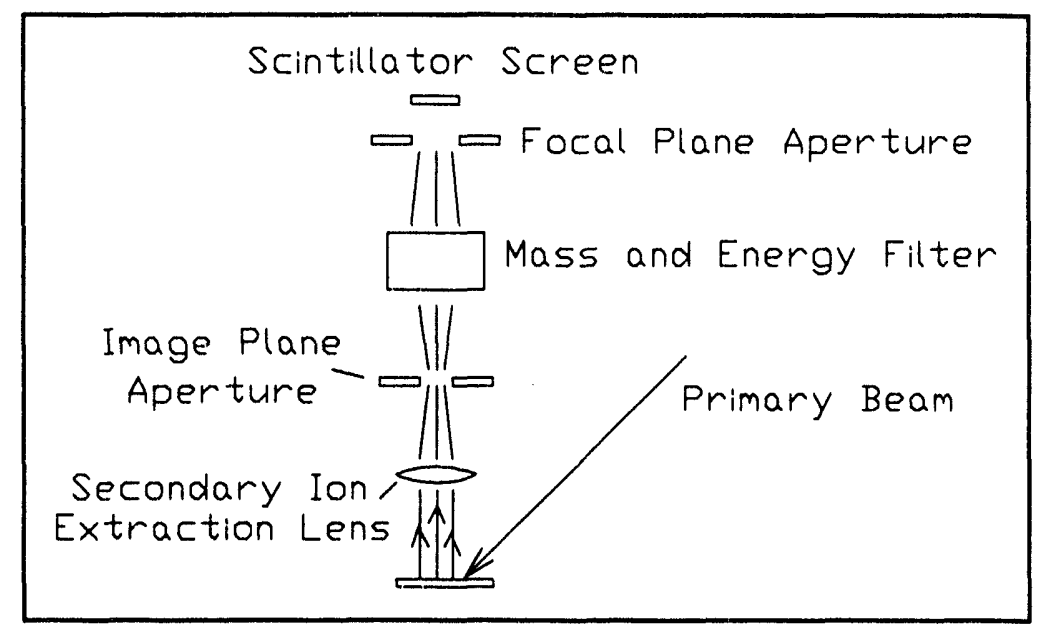

Figure 2: Direct imaging ion microscope ${ }^{15}$

dual microchannel plate. Unfortunately, spatial resolutions can only be obtained up to $10^{5}$ counts per second (cps) before the RAE becomes saturated.

Wizemann et al. ${ }^{11}$ have used a CAMECA IMS 300 ion microscope to study the emission characteristics of a thermal lithium ion source. The ion microscope had to be modified in order to place the source at the target area of the ion microscope and also to heat the lithium source to produce thermionic emission of the ions. The ion source was stepwise heated and the emission images of both lithium and potassium ions were observed. This is similar to what will be done with the instrument presented in this thesis. However, in the imaging instrument in this paper the ions will not be mass resolved. This should lead to better sensitivities since the ions will have to go through fewer ion optics. On the other hand, Thorne et al. ${ }^{12}$ have modified a CAMECA IMS $3 f$ ion microscope and been able to obtain images with less than $10^{3} \mathrm{cps}$. The improved sensitivity was accomplished by using a more sensitive video camera, a Nocticon instead of a Vidicon. This improved the sensitivity by $10^{2}$ to $10^{3}$. A similar increase in sensitivity could be expected by replacing the standard single micro channel plate with a chevron style micro channel plate. In addition to the camera, high speed image processing equipment was integrated into the system allowing the images to be averaged and integrated over time, thus further increasing the sensitivity of the instrument.

\section{Scanning lon Microprobe}

Another imaging instrument is the scanning ion microprobe which was under construction in 1964 and reported by Helmut Lieble in $1967^{13}$. Unlike the ion microscope, the primary beam in the scanning ion microprobe is microfocused ( $1-3 \mu \mathrm{m}$ in diameter) onto the sample. Deflectors raster the primary beam across the surface of the sample. The secondary ions are focused into a mass filter and then projected into a Faraday cup or an electron multiplier detector. Qualitative information is obtained by modulating the electron beam in a cathode ray tube (CRT) with the output of the detector and synchronizing the electron beam rastering with the primary beam of the ion microprobe. The image is formed on the phosphor screen of the CRT similar to the way pictures are formed for television. Quantitative information about the ion densities can be obtained by using an electron multiplier type of detector to record the ion counts for each point scan and storing the information in a 
computer. Quantitative images of the sample can later be produced from this information. A schematic view of a scanning ion microprobe is shown in Figure 3. The pre filter shown was used in the original ion microprobe mass analyzer (IMMA) designed by Helmet Liebl ${ }^{13.14}$. It was used to reduce the contaminants as well as the multiply charged ions from the primary beam. In the microprobe, the images have been induced by the ion current intensity as opposed to the microscope which provides a direct image of the ion current densities. One of the disadvantages of the ion microprobe is that the time required to obtain an image can be orders of magnitude longer than the direct imaging ion microscope resulting in unwanted and possibly intolerable damage caused from the sputtering process.

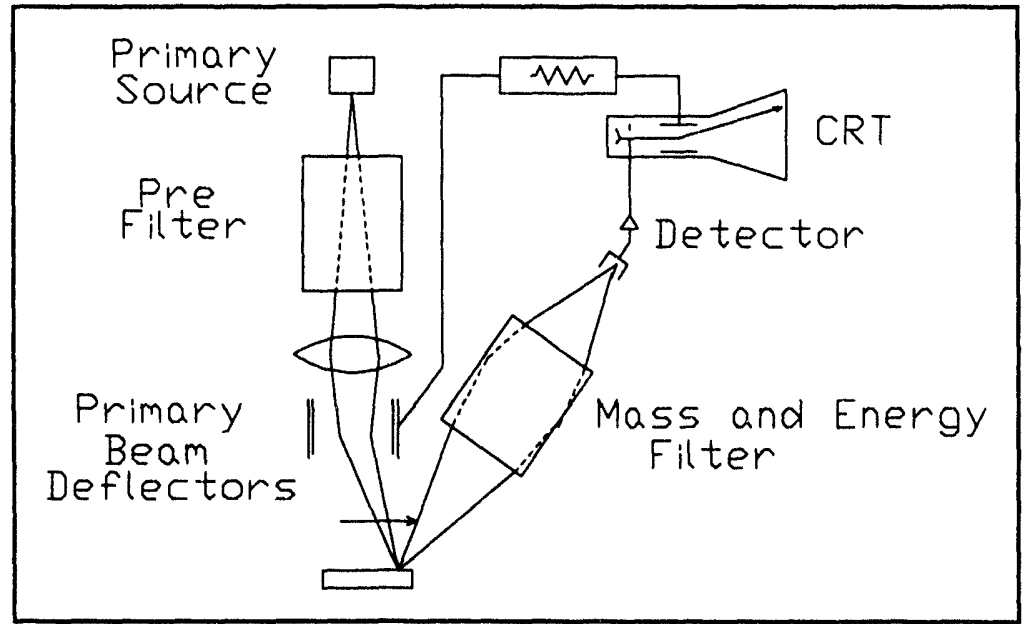

Figure 3: Scanning ion microprobe ${ }^{14}$

\section{Direct-Imaging Scanning Ion Microprobe}

A third type of imaging SIMS instrument is the direct-imaging scanning ion microprobe which was reported by McHugh et al. ${ }^{15}$. The instrument utilizes principles from both of the previously described imaging instruments. However the image formation is not direct in the same sense as the ion microscope. The sample is bombarded by a large diameter primary beam as in the ion microscope. Then an immersion lens is used to extract the secondary ions and focus them into a global image of the source. A field aperture is placed in the focal plane and then the secondary image is rastered across this aperture. The ions that pass through the small aperture are then mass analyzed, separated and detected in a Faraday cup or an electron multiplier detector. Like the ion microprobe, the output of the detector is used to modulate the beam intensity of the electron beam in a CRT. The secondary sweep plates are synchronized with the rastering of the CRT and a mass resolved image is formed on the screen of the CRT. The difference between this and the ion microprobe is the fact that the secondary sweep, as opposed to the primary sweep, is synchronized with the CRT scan. The obvious advantages of this system is that there is no need for any elaborate ion optics for the primary beam and only a single ion lens is needed for image formation of the secondary ions ${ }^{8}$. Also, since a global image of the sample is formed by irradiating the sample with a large primary beam, the time for image formation should be similar to that of the ion microscope. A schematic showing the basic elements in the direct-imaging scanning ion microprobe are shown in Figure 4. 


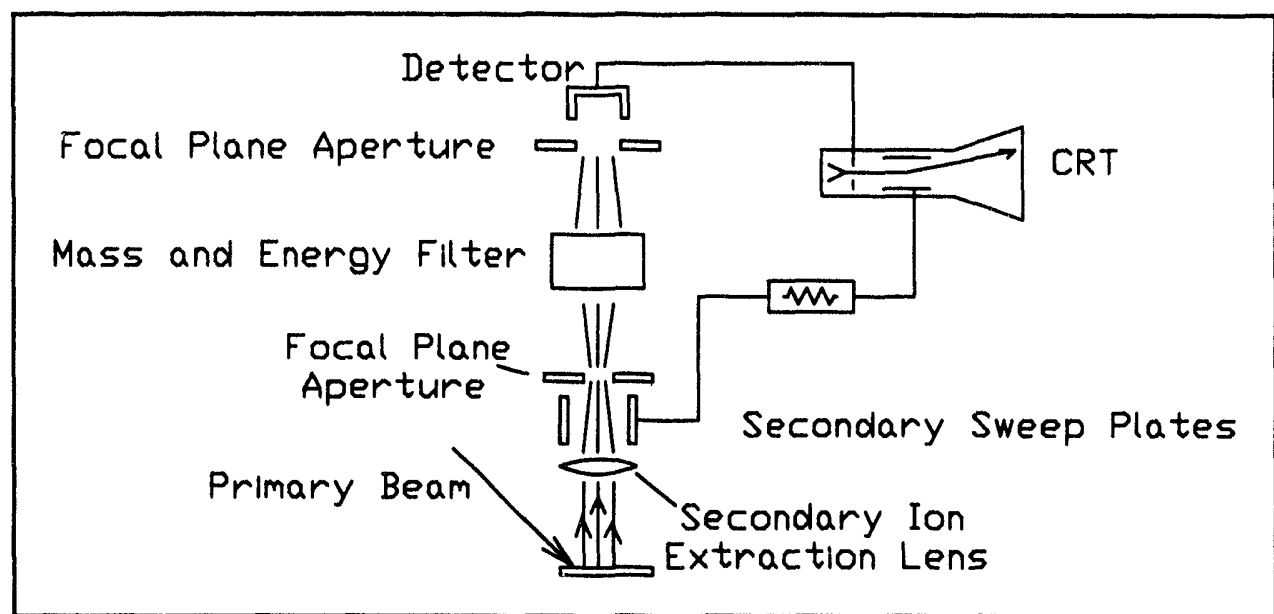

Figure 4: Direct-imaging scanning ion microprobe

\section{Imaging Instrument (This Paper)}

Of the imaging techniques that have been used in other instruments, only two could be considered for this instrument. The most straight forward, and the one that was used for this instrument, is the direct imaging technique similar to the field-ion microscope. However, ion emission will not be based on field emission. Instead, the ions will be produced by thermionic processes, at least in the original experiments. The ions will be electrostatically accelerated and focused onto a microchannel plate equipped with a phosphor screen for immediate viewing of the images. A more complicated method which should work in theory would be to use an imaging scheme similar to that used in the direct-imaging scanning ion microprobe. However, it is doubtful that the resulting complexity of the instrument as opposed to the performance that might be achieved would warrant the extra effort involved in the design and construction of the instrument. An obvious advantage in the direct imaging method is that the distortions of the image will be minimized since the ion beam will be subjected to fewer ion optics. In the scanning method, great care would have to be exercised in both the placement of the scanning deflectors and the rastering of the beam over the aperture so not to distort the image of the ion emission from the surface. The direct imaging method should also provide higher resolutions for similar reasons. It is expected that the imaging instrument will be an addition to the scientist's arsenal of tools for gathering qualitative information about the ion emission processes and lead to a better understanding of these phenomenon. The information, identifying the areas of higher (or lower) ion emission from the surface, can be used to direct the investigator to further analyze these areas in an attempt to determine why the ion emission is enhanced in some areas as opposed to others. 
INTENTIONALLY LEFT BLANK 


\section{CHAPTER III \\ INSTRUMENT DESIGN AND CONSTRUCTION}

The purpose of this project was to construct an instrument for gathering qualitative information pertaining to the spatial origin of emission of ions from ion sources. In this instrument the ions will be thermionically emitted and then electrostatically accelerated and focussed onto a microchannel plate detector equipped with a phosphor screen for immediate viewing of the current densities of the ions being emitted from these sources. Since the ion sources that will be studied with this instrument will be pure ion emitters, no mass discrimination is necessary. The instrument was designed to be versatile and capable of analyzing a variety of ion sources. Reversible power supplies were used, allowing both positive and negative ions to be studied. The source mount was designed around the National Bureau of Standards (NBS) filament hat (mount). Therefore, any source that works in the NBS filament hat will also fit in this instrument. Different ion source designs are readily adapted to this configuration. The ion lens is constructed of standard NBS components and can be easily installed and removed from the instrument, allowing the ion optics to be adapted for different experimental conditions. Hopefully this imaging instrument will provide one more tool that can be used, after rigorous testing of these ion sources to confirm that only one species of ions are being emitted, to help in explaining the emission process.

The following subsystems were integrated together to develop the instrument: 1) the vacuum system, which includes the vacuum chamber and pumps; 2) the ion beam, including the ion gun, detection system and deflectors; 3) the video and photographic cameras; 4) the data control system, including the computer control and signal conditioning circuitry. In this chapter we will describe the development of each of the components that went into the construction of the imaging instrument. When appropriate, brief descriptions of the theories behind these components will be included to give a better understanding of the entire instrument. A detailed description of the signal conditioning circuit is given in Appendix A. Appendix B contains the User's Manual for the computer control system.

\section{Vacuum System}

\section{Vacuum chamber}

The main vacuum chamber was designed around an old JEOL JMS-01BM-2 mass spectrometer. The old mass spectrometer was securely attached to a cast aluminum platform which was mounted on a steel table. In order to provide good stability, this existing support structure was used for the instrument. The source chamber was securely attached to the aluminum platform and consequently the source chamber became the corner stone for constructing the imaging instrument. Instrument assembly was simplified since the source chamber was already equipped with an isolation valve and electrical feedthroughs. However, one of the disadvantages associated with this choice was that the chamber's flanges used gold seals that did not facilitate the addition of standard vacuum components. Therefore custom adapter nipples were manufactured to complete the vacuum chamber. Another disadvantage was that the table upon which the instrument sat was large and required a substantial amount of lab space. An overall isometric view of the finished vacuum chamber can be seen in Figure 5. A $15.24 \mathrm{~cm}$. diameter view port was installed on the rear flange of the source 
chamber allowing the lens and source to be viewed while operating the instrument. The view port was necessary so that the temperature of the ion sources could be measured with an optical pyrometer. Another technique for measuring the temperature of the source would be to use a thermocouple. However, this would require additional electrical feedthroughs.

All of the components of the vacuum chamber were constructed using 304 stainless steel (SST). 304 SST is a standard material used in vacuum components because of its high strength and ability to withstand the temperature fluctuations associated with vacuum work during bake-out procedures. Stainless steel also can be machined to a smooth surface and does not oxidize. Oxidation creates a rough surface increasing the surface area for gases to adhere to, causing outgassing problems $^{16}$.

\section{Pumping system}

The source chamber is maintained under vacuum by a Pfeiffer Balzers TPU-170 turbomolecular(turbo) pump. Turbomolecular pumps are reliable mechanical pumps which are used extensively in industry and research applications due to the ease at which turbo pumps can achieve hydrocarbon-free high vacuum ${ }^{17}$. A turbo pump was chosen for this portion of the instrument since it could rapidly evacuate the source chamber, thus speeding up the turn-around time between source changes or ion optic modifications. The pressure in the source chamber has been observed as low as 2 $\times 10^{-8}$ Torr, but during normal operation it is maintained at or below $2 \times 10^{-7}$ Torr.

Turbo pumps can not discharge directly to atmospheric pressure and normally require an exit pressure of 76 millitorr or less ${ }^{17}$. An Edwards E2M-8, two-stage rotary vane mechanical pump was used as the fore pump for the turbo pump and also to provide initial evacuation of the chamber. In order to reduce the vibrations introduced by the roughing pump, the pump was placed on a piece of foam on the floor and the connection to the turbo pump was made with a rubber hose.

One problem associated with the use of mechanical pumps is that when the system is allowed to vent through the rough pump, as in the event of a power outage, oil vapors can back-stream into the vacuum chamber and contaminate the inside of the chamber. After this happens, it can take a long time for the oils to outgas and achieve good vacuum. In order to help prevent back-flow through the rough pump, a Varian pneumatic valve was placed on the inlet to the rough pump. When power is removed from the roughing pump, the valve closes and blocks the path for the oil vapors to enter the vacuum chamber. Normal venting of the system was provided through a dry nitrogen line attached to the turbo pump. When the chamber is opened to the atmosphere, the nitrogen line maintains a positive pressure inside the vacuum chamber to reduce the contaminants, such as water vapor, from entering the chamber.

Turbomolecular pumps consist of a series of high speed rotor and stator blades. Due to molecular collisions with the blades and frictional losses in the bearing system a great deal of heat can be generated inside the turbo pump. Water circulation was used to provide the cooling for the pump.

The flight tube portion of the vacuum chamber is held under vacuum by the use of a PerkinElmer $270 \mathrm{l} / \mathrm{s}$ ion pump. An ion pump was chosen for two main reasons. First, the pumping action in an ion pump is not mechanical. Therefore, they do not introduce any vibrations into the system. Instead of removing the gas molecules from the system, ion pumps convert the molecules from the gas phase to the solid phase ${ }^{18}$. This phase transformation occurs in two ways: 1) active gases react with 


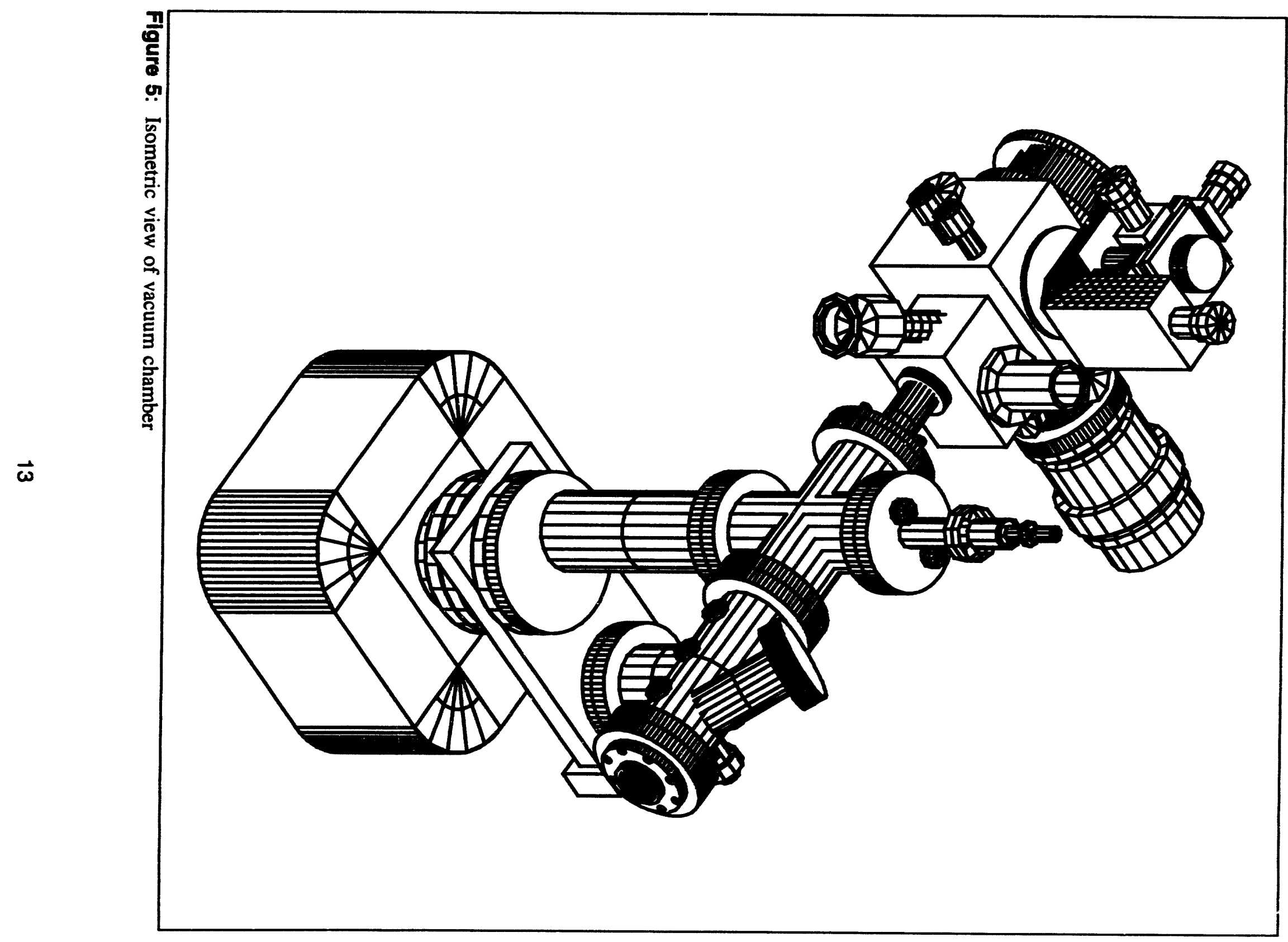


getter material and form stable compounds; and 2) ions or neutral species of molecules become buried within the surfaces of the ion pump. The second reason for using an ion pump is that they provide an oil free environment. This is desirable for the flight tube portion since it contains a critical and expensive component vital to the operation of the instrument, the microchannel plate(MCP).

Permanent damage could occur to the MCP if the surfaces were contaminated from oil. When the instrument is not operating, the flight tube is isolated from the source chamber, thus eliminating the possibility of venting through either of the mechanical pumps.

Vacuum pressures range between $1 \times 10^{-8}$ and $5 \times 10^{-8}$ Torr during operation of the instrument. The ion pump can not be turned on until the pressure in the flight tube is at or below $2 \times 10^{-5}$ Torr. Therefore, before the ion pump can be turned on, the flight tube is roughed out by the use of the combination of the Edwards roughing pump and the turbo pump.

The ion pump is controlled by a Perkin-Elmer Digitel 500 power supply and ion pump controller. The controller serves several functions such as supplying high voltage to the ion pump, monitoring current drawn by the ion pump, and acting as a pressure gauge. Ion pumps are selfregulating, that is, as the pressure goes down so does the current drawn by the pump. Furthermore, the relationship between current and pressure is very nearly linear. The controller utilizes this linear relationship and the controller is used as a pressure gauge for the flight tube portion of the instrument. The controller also acts as a protection circuit for the ion pump. If the pressure becomes too high the current drawn by the ion pump will increase and the power dissipated by the pump can cause irreparable damage. When the current drawn by the pump becomes too large, the controller will automatically shut the power off to the pump.

The ion pump was mounted underneath the cast aluminum platform on a screw support stand. A gate valve was installed on top of the pump so that the ion pump could be isolated during venting procedures.

\section{Ion Beam}

The ion beam for the instrument required several components: the ion gun consists of the ion lens, the source manipulator, ion source, and the electronics to power the lens elements; following the ion gun are the deflectors, which steer the ion beam for positioning on the microchannel plate or which deflect the ion beam off the MCP screen(dumping). The ion beam is detected by either a Faraday cup detector or the MCP.

\section{Ion gun}

Ion lens. The ion lens used in this system was a gun that was first designed and reported by Delmore, Appelhans and Dahl as an autoneutralizing neutral molecular beam gun'. The lens was designed to minimize the ion residence time within the lens and also to maintain reasonable focusing properties, thus allowing a small beam diameter. The lens design turned out to have good imaging qualities, as could be seen on the MCP. The lens design was modified slightly to improve its imaging capabilities. This will be covered in more detail in the system testing section.

The lens was constructed using parts supplied by the National Bureau of Standards(NBS). In order to mount the standard components, the lens mount used in the JEOL source chamber had to be 
modified. The modification consisted of hiving a rectangular piece of stainless steel made to fit where the adjustable exit slit was in the original instrument. The rectangular piece allowed a standard final aperture mount to be fastened to the mounting bracket. The lens mount has a cylindrical end which mates in a hole and has " $\mathrm{L}$ " shaped wings on the top and bottom which fit into spring clips to secure the lens assembly. The lens assembly can be seen in Figure 6.
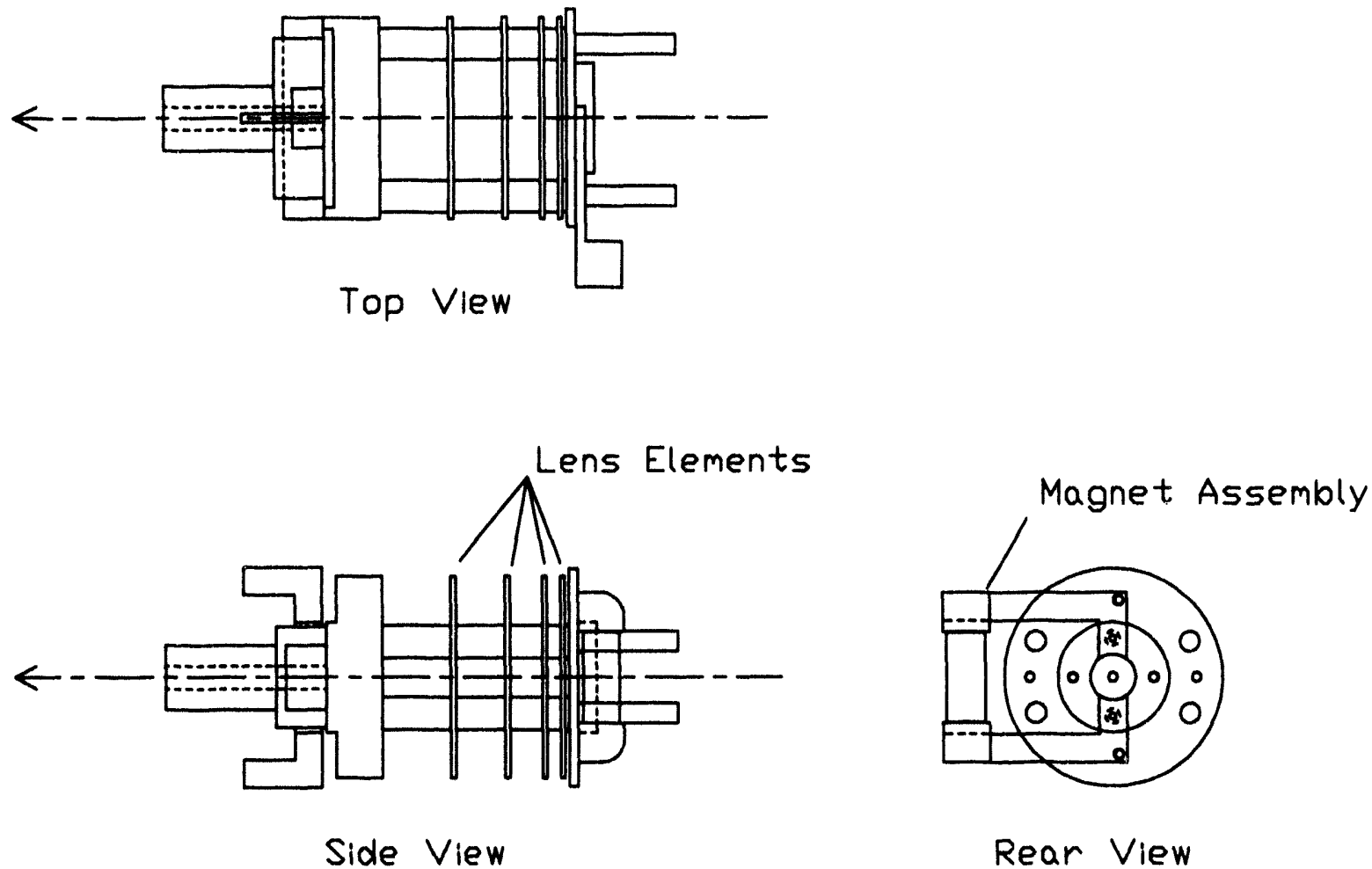

Figure 6: Ion lens assembly

The lens is a cylindrical design. The lens elements are round stainless steel plates with circular apertures in the center. The elements mount over four ceramic posts which are $8.89 \mathrm{~cm}$ long. Ceramic spacers are used to provide the offset between the different elements. The assembly is compressed together by placing collars over the ceramic posts, held in place by set screws, on the outside of the shield plate and then tightening the posts to the final aperture mount.

A magnet assembly is mounted on the shield. The magnets are needed when imaging anions. Since the voltages used to accelerate and focus the anions will also accelerate the electrons, a magnetic field is used to cause the electrons to spiral off, thus preventing them from being accelerated in the lens.

Source manipulator. During the early stages of development of the instrument, the source was rigidly attached to the shield plate. Crude alignments of the source with the center of the ion optics were done by bending the filament to position the source. However, once the ion sources were mounted on the ion gun there was no method to reposition the source during imaging procedures. The area of the ion emitter surface that was visible during zooming was ultimately limited by the final position of the ion source relative to the rest of the ion optics. The deflectors could be used to 
reposition the image on the microchannel plate(MCP) but the area visible in the image could not be changed. To overcome this problem, the ion source was detached from the shield plate and suspended from a remote mounting assembly attached to an XYZ manipulator. The manipulator chosen was a Huntington model PM-275 XYZ precision manipulator. Micrometer movements provided a position resolution of $0.002 \mathrm{~mm}$. The top flange of the source chamber had to be adapted to have a $6.985 \mathrm{~cm}$ conflat flange to accommodate the flange on the manipulator. The manipulator sat directly above the ion lens and slightly in front of the rear plane of the shield plate. Therefore, the remote mounting assembly had to be designed and built to provide both vertical and lateral displacement of the source. In addition, the assembly had to (i) provide adequate stability so the images were not effected by vibrations; (ii) provide at least $10 \mathrm{kV}$ of isolation to ground; and (iii) allow easy exchange of ion sources.

The vertical displacement was accomplished by welding a $1.905 \mathrm{~cm}$ diameter stainless steel tube $27.94 \mathrm{~cm}$ long to a $6.985 \mathrm{~cm}$ diameter conflat flange. A tube was chosen to provide a rigid support for the source. The XYZ manipulator was electrically isolated from the source by a half cylindrical piece of vespel installed between the stainless steel tube and the final mounting hardware for the ion source. A $1.905 \mathrm{~cm}$ diameter hole was placed in one end of the vespel allowing the piece to slide onto the support tube. The vespel piece was clamped to the tube by machining a slot in one end to permit the hole to be compressed around the tube. A rectangular support bracket was used to align the source with the ion lens. Since this support bracket would have to be removed during lens removal, a stainless steel end piece was mounted to the end of the vespel piece and the support bracket was attached to the end piece with three screws. This was done to alleviate any fatigue on the threads that were placed in the vespel. A filament hat mounts to the support bracket. The filament hat was designed so that the sources that fit the NBS filament hats will also fit in this system. However, NBS filament hats will not interchange with this assembly. The overall design of the mounting assembly can be seen in Figure 7.

Ion source assembly. The ion sources used for this project utilize surface ionization to produce ions. Typically, surface ionization is accomplished by depositing a small amount of material on a suitable filament and then heating the filament until ions are emitted. Delmore et al. have reported a tube ion source in which the ion emitting material is contained in a refractory metal tube (rhenium, stainless steel or palladium). The tube acts as a reservoir of material allowing the source to be analyzed before and after it is used to determine any changes that have occurred. The identification of these changes may lead to a better understanding of the ion emission process ${ }^{19}$. A version of the tube source design was used for most of the imaging done for this thesis.

The first step in constructing a tube source is to cut a tube of stainless steel or rhenium $4 \mathrm{~mm}$ long, $1.6 \mathrm{~mm}$ o.d., and $1 \mathrm{~mm}$ i.d. After deburring the ends of the tube, the tube is placed in a special press where the emitter material can be packed into the tube and one end plugged with a small piece of wire. Next, two insulated posts are spot welded to a small cross member which holds them parallel to each other $7.5 \mathrm{~mm}$ center to center. A piece of rhenium ribbon is then spot welded between the ends of the two insulated posts. Finally, the tube is spot welded to the rhenium ribbon. Along with supporting the tube, the rhenium ribbon is the filament that heats the tube to cause ion emission. A sketch of the tube source design is shown in Figure 8 . The source assembly fits into the filament hat and is held in place by two set screws.

Two different ion sources were analyzed for this project. The first source was a cesium $\left(\mathrm{Cs}^{+}\right)$ cation emitter. The emitter material was cesium zeolite A. Type A zeolite is a synthetic alkali 

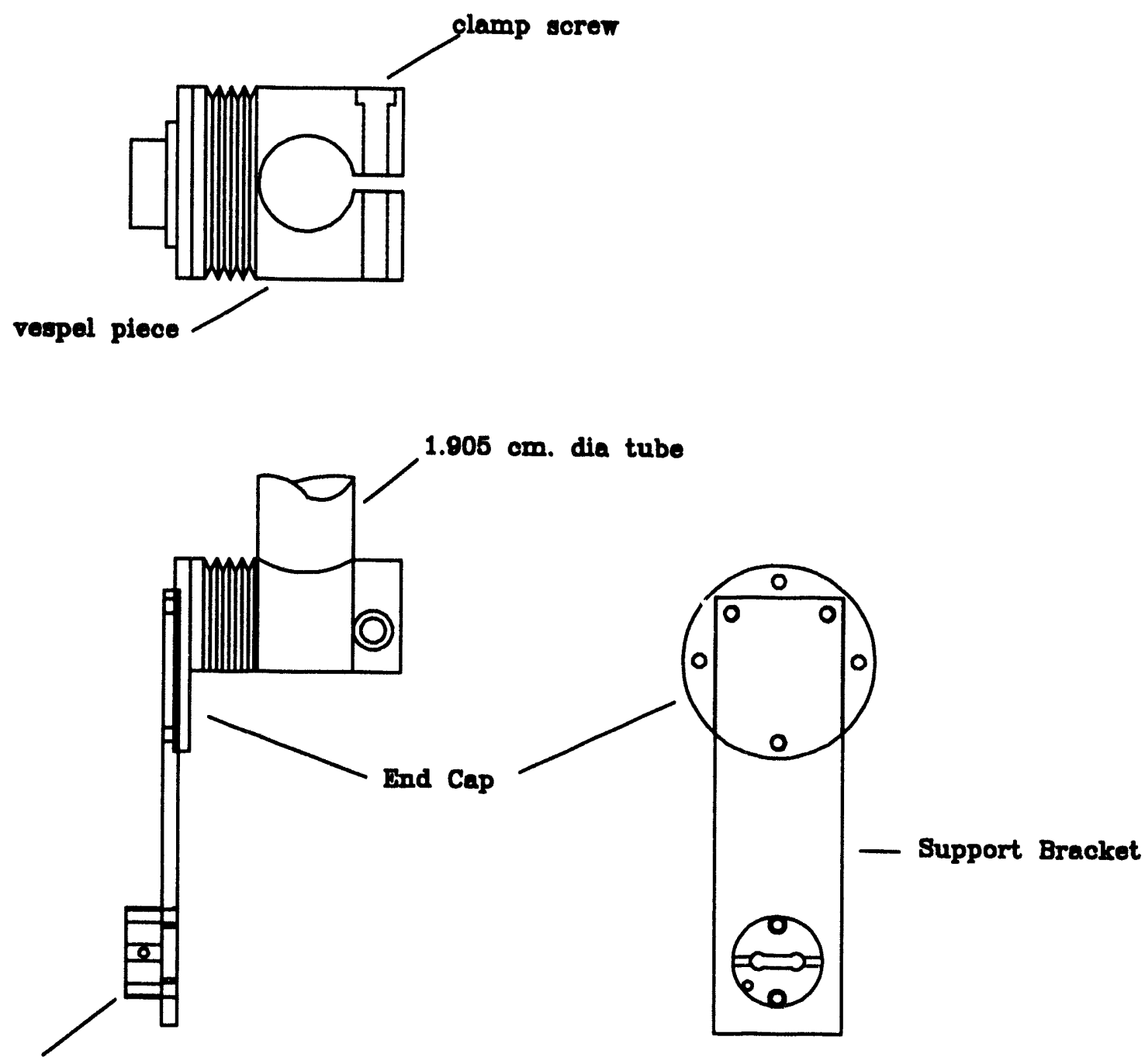

Filament Hat

Figure 7: Ion source mounting assembly

aluminosilicate, in which after proper stirring and percolation procedures, ion exchange can occur and produce pure ion emitters $(>99 \%)$ of cesium, sodium, lithium and potassium ions ${ }^{20}$. The second source was a perrhenate $\left(\mathrm{ReO}_{4}^{-}\right)$anion emitter.

Ion gun electronics. The lens elements are operated between 5 and $10 \mathrm{kV}$ in both positive and negative polarities depending on the polarity of the ions being imaged. Independent power supplies were used to provide the focusing voltages on the lens elements. Five Spellman $\pm 10 \mathrm{kV}$, and one Bertan $\pm 5 \mathrm{kV}$ supplies were used to supply these voltages. All of the supplies were capable of being manually controlled, via the front panel controls, and also being remotely controlled with an analog voltage signal. 


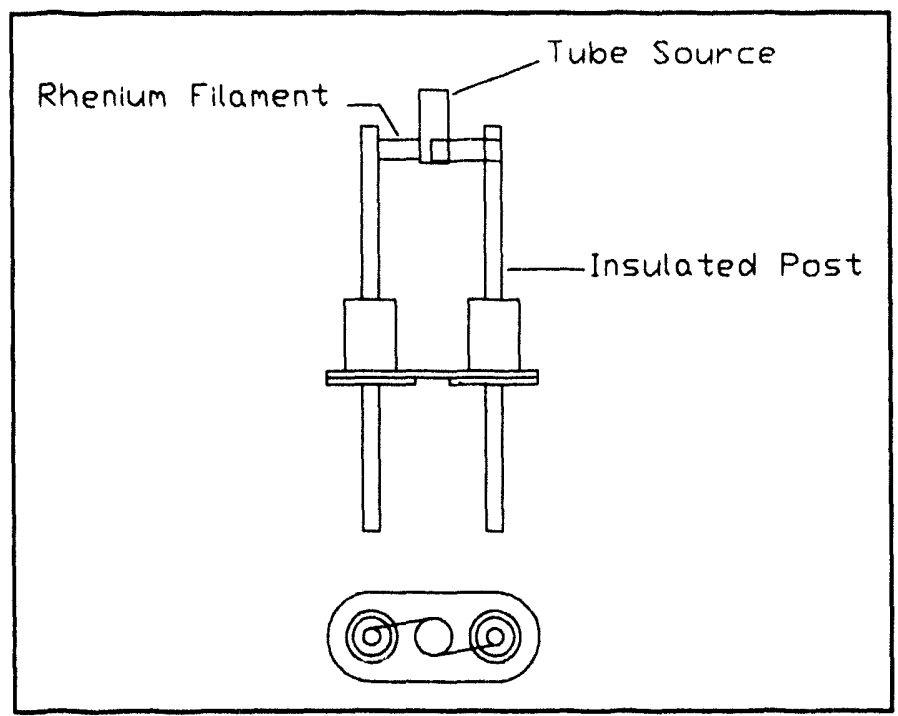

Figure 8: Tube source assembly

The ion source is held at the highest potential and the exit aperture is at ground potential. The filament has a small resistance, less than one ohm, therefore, a power supply capable of supplying a small voltage and a large amount of current was needed. In addition, the power supply had to be floated at high voltage. An isolation transformer was used to supply the 110 volt ac for the supply. Then the power supply was floated at high voltage by tying its ground to a Spellman $\pm 10 \mathrm{kV}$ supply. The power supply was electrically isolated by mounting it in a plexiglass chassis.

It is inherent in high voltage charged particle acceleration and focusing that an occasional arc will be drawn inside the lens assembly. Therefore, the supplies need to be protected from these arcs. Experience has shown that the Spellman and Bertan supplies are fairly robust and capable of handling occasional arcs. Hence no external arc protection was provided for these power supplies. Since the ion source and filament are a probable source of arcing, the filament power supply needed some protection. This protection was provided by installing a pi-filter network on both the positive and negative legs of the filament supply. The design is one that has been used on other supplies in the mass spectrometry lab. A schematic of the filament supply configuration is shown in Figure 9.

The temperature of the source is proportional to the current flowing in the filament. Therefore, a meter was installed to monitor the filament current. A $0.1 \mathrm{ohm}$ precision resistor was mounted in series with the filament, and the voltage developed across the resistor was fed directly into a small liquid crystal display. A small 5 volt supply, also floating at high voltage, was used to power the display.

\section{Detection system}

After the ion beam exits the ion lens, it enters a deflection electrode region, followed by a drift region, then it encounters two detection systems. The Faraday cup can be inserted into the beam line to measure the intensity of the ion beam, or it can be removed from the beam line, and after a second drift region the ion beam will impinge on the surface of a microchannel plate detector(MCP). The 


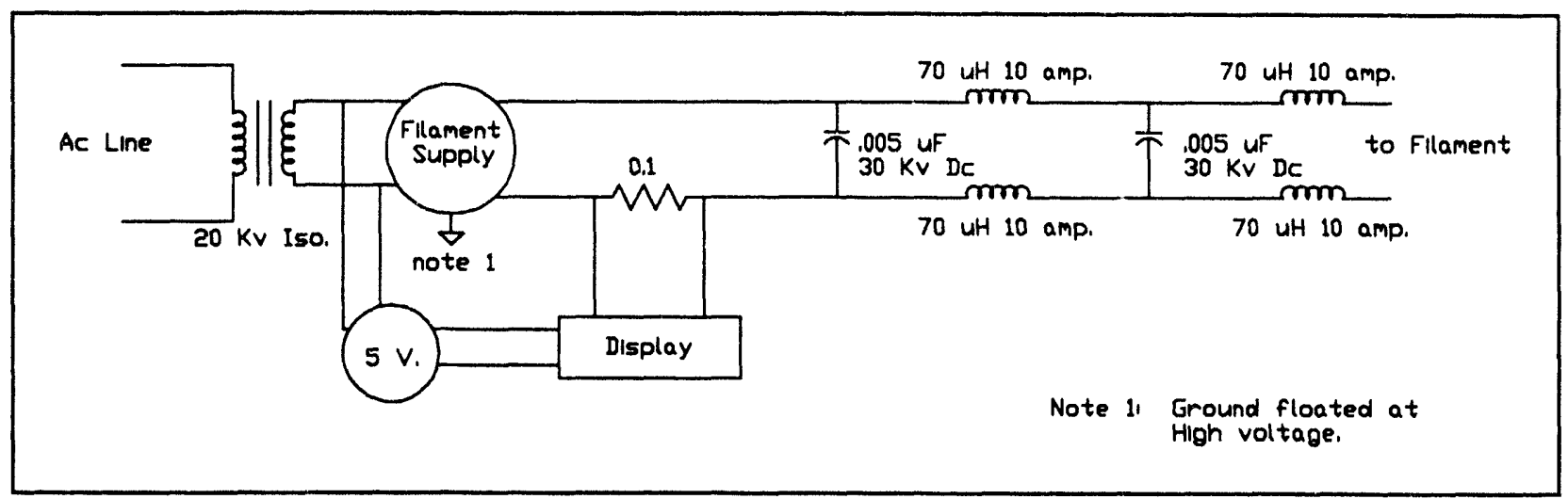

Figure 9: Filament supply schematic

MCP is a critical component in the imaging instrument because it converts the ions into visible images and is used to evaluate the fine focusing of the ion images.

Faraday cup. The first method that was used to detect and measure the ion beam current was a Faraday cup. This device was capable of being moved in and out of the ion beam with a vertical linear motion manipulator. The Faraday cup is used for two purposes: 1) to detect and measure the ion current; and 2) protect the MCP by blocking the ion beam. However, the MCP was usually protected by deflecting the beam off the screen.

The theory of operation of a Faraday cup is simple. A conducting cup is placed in line with the ion beam to stop the ions. As the ions are stopped, a charge builds up on or near the surface of the cup. When the cup is connected to an electrometer, the electrometer will try to neutralize the charge build up on the cup. Depending on the polarity of the beam, current will either flow to or from the electrometer, resulting in a positive or negative current reading ${ }^{21}$. Erroneous readings can be obtained in the event of secondary electron emission in which the electron escapes from the cup. If a secondary electron escapes, the net charge that is built up on the cup will be too positive and not reflect the true ion intensity in the system. The Faraday cup used in this instrument is similar to the design of the Faraday cup in the charge monitoring instrument designed by Scott Beck ${ }^{21}$. In this design several features are employed to minimize the probability of electrons escaping from the cup and will be discussed as the design is presented.

The Faraday cup was constructed using Kimball Physics components. The main cup was a stainless steel cylinder $25.4 \mathrm{~mm}$ long and $i 2.7 \mathrm{~mm}$ in diameter. One end of the cylinder is ground off at an angle of 45 degrees and a thin Molybdenum sheet was spot welded to close this end of the cup. Molybdenum was used because it has a lower affinity for producing secondary electrons ${ }^{21}$ than other metals. By angling the backplane of the Faradey cup at 45 degrees, the reflected ions and resulting secondary electrons are less likely to be directed towards the opening of the cup. The cylinder was spot welded to a $20.32 \mathrm{~mm} \times 20.32 \mathrm{~mm}$ square stainless steel plate which had a $12.7 \mathrm{~mm}$ diameter aperture.

A suppressor grid was placed $2.54 \mathrm{~mm}$ in front of the entrance to the cup. The suppressor was made of a $20.32 \mathrm{~mm} \times 20.32 \mathrm{~mm}$ square stainless steel plate with a $12.7 \mathrm{~mm}$ diameter aperture. A stainless steel mesh was spot welded across the aperture to smooth the voltage field. By biasing the suppressor at -50 volts the majority of the stray electrons can be repelled back into the cup. 
Finally, a ground plane was introduced $5.08 \mathrm{~mm}$ in front of the suppressor grid. The ground plane is necessary to minimize any defocusing of the beam caused from the voltage gradients introduced from the suppressor grid and the charge build up on the cup. The ground plane was made from a $20.32 \mathrm{~mm} \times 35.56 \mathrm{~mm}$ stainless steel rectangular plate. One row of holes was trimmed off to make the length $25.4 \mathrm{~mm}$ so it did not protrude down any further than was necessary when removed from the beam line. The plate also had a $12.7 \mathrm{~mm}$ diameter aperture. A similar plate, which did not have an aperture in it, was placed at the other end of the cup. Angle brackets were used to secure a $35.56 \mathrm{~mm} \times 35.56 \mathrm{~mm}$ square plate to the front and back plates for mounting purposes. A sketch of the design of the Faraday cup is shown in Figure 10.

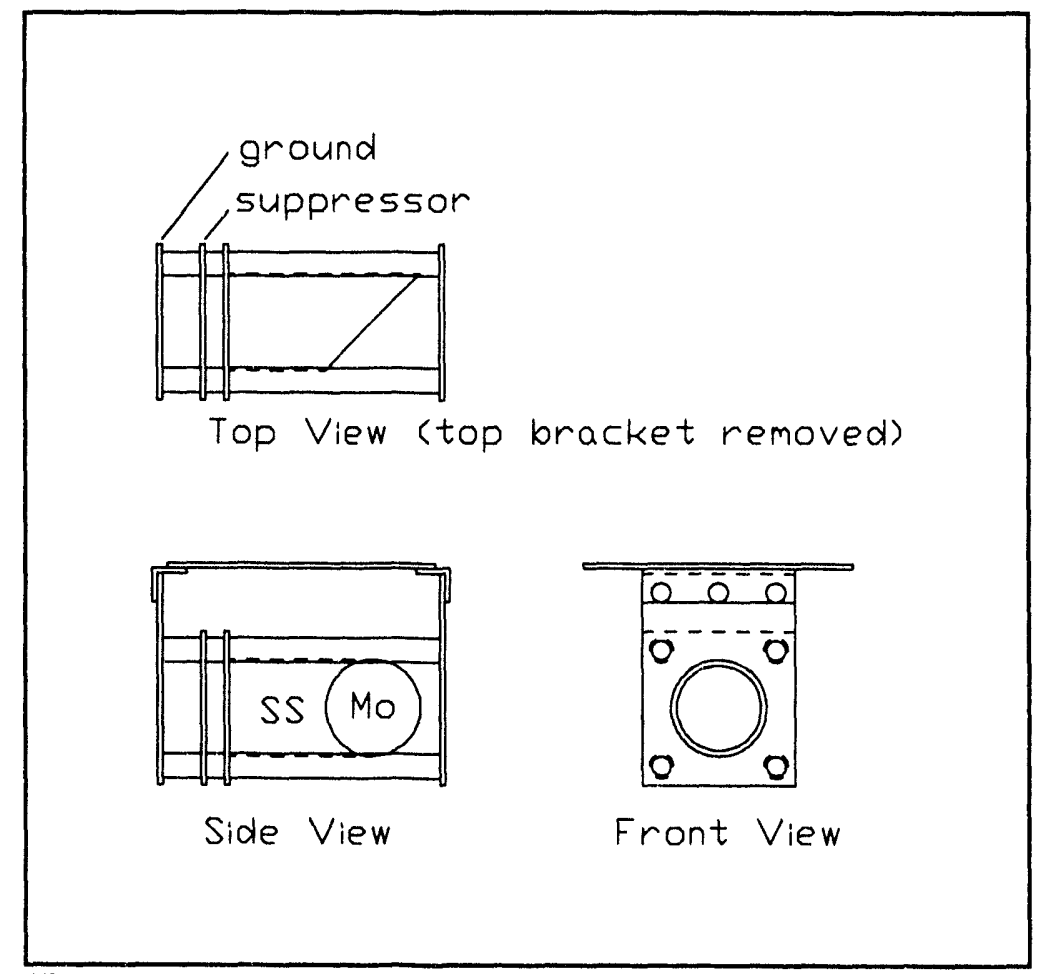

Figure 10: Faraday cup assembly

The cup was secured to a linear manipulator with two 10-32 threaded rods approximately 12.7 $\mathrm{cm}$ long. The manipulator is spring loaded and provides $7.62 \mathrm{~cm}$ of travel, allowing the Faraday cup to be easily withdrawn from the beam line. The electrical connections were made by spot welding wires to the suppressor and to the plate on the cup. Springs were then placed between the wires on the electrical feedthroughs and the wires on the cup elements. The springs allowed the cup to be retracted and reinserted into the beam while keeping the electrical connections out of the way.

The Faraday cup was connected to a Keithley 617 programmable electrometer. This is a highly accurate electrometer with a resolution down to $100 \mathrm{aA}\left(10^{-18} \mathrm{amps}\right)$. Programming of the electrometer can be performed using a IEEE-488 interface.

Microchannel plate. The second method of detecting the ion beam was the microchannel plate, MCP. The MCP is a Galileo model $6040 .^{22}$ The MCP serves two purposes: 1 ) to detect the presence of an ion beam; and 2) for imaging the ion source. MCPs are used in a variety of 
applications, including night vision systems, mass spectroscopy, and scanning electron microscopy.

An MCP is a collection of thousands of tiny electron multipliers. The electron multipliers are small diameter cylinders, $10-100 \mu \mathrm{m}$ in diameter, made of lead glass. The diameter of the channels in this channel plate are $10 \mu \mathrm{m}$ and are spaced $12 \mu \mathrm{m}$ center to center. The glass is treated in such a way as to enhance the secondary electron emission characteristics and also to render the channel walls semiconducting to enable charge replenishment from an external power supply ${ }^{23}$.

A bias voltage of up to 1000 volts is placed across the array of microchannels. When an ion or other form of primary radiation strikes the inside surface of one of these channels with sufficient energy to cause the release of secondary electrons, these electrons are accelerated down the channel. As the electrons travel down the channel, some of them will collide with the inside surface and release more secondary electrons. This cascading effect continues until the electrons exit the channel.

Electron multiplication factors of $10^{3}$ to $10^{4}$ per MCP can be achieved. After the electrons exit the channels, they are further accelerated by a potential of up to 5000 volts which is applied to a phosphor screen. The phosphor is deposited on a fiberoptic substrate allowing the image to be viewed ${ }^{24}$. A schematic showing the arrangement and bias voltages for a single microchannel plate is shown in Figure 11.

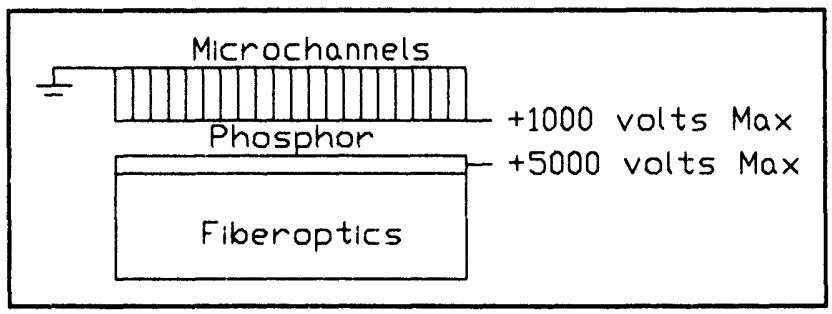

Figure 11: Microchannel plate schematic

Earlier experiments performed by Delmore and Appelhans ${ }^{25}$ on the SIMS instrument demonstrated that the bias voltages associated with the MCP distort the ion beam near the edges. Therefore, a grounded shield was installed inside the reducing flange, directly in front of the MCP. The shield helps reduce the field gradients introduced by the electrical connections to the MCP.

The shield was constructed using a piece of stainless steel shim stock cut out in the shape of a doughnut. The inside diameter was $44.45 \mathrm{~mm}$ and the outside diameter was $63.5 \mathrm{~mm}$. Three 10-32 hex nuts were spot welded at approximately 120 degree intervals near the outside perimeter and set screws were used to secure the shield inside the reducing flange.

\section{Deflectors}

Beam steering deflectors were needed in the system to allow the ion beam to be moved around on the micro channel plate (MCP), where the image was observed. In order to minimize the need for deflection during ion imaging, the image intensifier was mounted directly in line with the ion source. In addition to providing small diversions of the ion beam, the deflectors were used to deflect the ion beam away from the MCP screen when the image was not being viewed. This was necessary to prevent screen burn on the MCP.

Several configurations for electrostatic deflectors are possible. The simplest design is to mount two flat plates parallel to each other. By creating a voltage differential between the two plates 
a charged beam can be deflected due to the electrostatic forces. Typically two sets of deflectors are used, one for the $\mathrm{x}$ and one for the $\mathrm{y}$ directions. Generally the two sets of deflectors are not coplanar. However, a quad arrangement was used in this instrument. The quad consisted of two sets of deflector plates whose effective deflection region along the path of the ion trajectories coincided with one another. Simion ${ }^{26}$, an ion optics modelling program, was used to analyze this type of arrangement. The field gradients near the center of the quad are fairly linear and toward the edges have a pincushion shape. Unfortunately, the fringing effects that are present at the ends of the deflectors can not be modelled with existing programs like Simion. By placing the deflectors close to the final aperture of the ion lens the beam can be steered while it still has a small cross-sectional diameter, allowing it to remain in the linear regions.

In order to mount the deflectors, a custom nipple was constructed. The overall design is shown in Figure 12. The nipple adapts the gold seal flange to a $15.24 \mathrm{~cm}$. conflat flange, and provides 4 electrical feedthroughs for the deflector control voltages. A $1.9 \mathrm{~cm}$. diameter stainless steel tube is held in place at one end with a teflon plug which fits snug around the tube and inside the nipple and at the other end with a stainless steel support bracket. The support bracket gives mechanical stability and electrically ties the $1.9 \mathrm{~cm}$ tube to ground. Four Kimbal Physics type C ceramic tubes $^{27}(3.15 \mathrm{~mm}$ o.d., $1.65 \mathrm{~mm}$ i.d. and $15.24 \mathrm{~cm}$ long) are mounted between the $1.9 \mathrm{~cm}$. tube and the outer $3.81 \mathrm{~cm}$. diameter tube. The ceramic tubes provide electrical isolation and support for the wires that supply the voltages to the deflectors. The deflectors were mounted inside the 1.9 $\mathrm{cm}$. tube near the end, and the tube was positioned as close to the isolation valve as possible. This provided a convenient way of mounting the deflectors while also allowing the deflectors to be placed as close to the final aperture as possible.

When adjusting the voltages on the deflectors, either one plate can be held at ground potential and then the other plate adjusted or both plates can be adjusted simultaneously one going positive and the other negative. In the latter case, the main axis of the beam line is maintained at ground potential. By adjusting the deflectors in this manner, the defocusing and lensing effects introduced by the deflectors can be reduced.

The deflector voltages were supplied from a Spectrum Solutions TD-9500 power supply. This power supply had 9 separate $\pm \mathbf{5 0 0}$ volt outputs. Each output could be manually adjusted or computer controlled with a \pm 10 volt analog control signal. When in the computer control mode, the voltages could only be adjusted via an external control signal.

\section{Video System}

A video system was added to the instrument to make it easier to view the images during operation. The system consisted of a Cohu 5100 series monochrome, low light level, closed-circuit video camera, and two video monitors. One video monitor was placed near the computer terminal so the operator could see the images while using the computer interface. The other monitor was placed near the source chamber so the images could be monitored while the source was being repositioned with the remote manipulator.

The camera has a silicon intensified target (SIT) vidicon image tube. SIT tubes provide good sensitivity to low light levels while also providing good resolution (600 lines horizontal at the center and 350 lines vertical ${ }^{28}$ ). The camera is also equipped with a remote control unit for adjusting the 


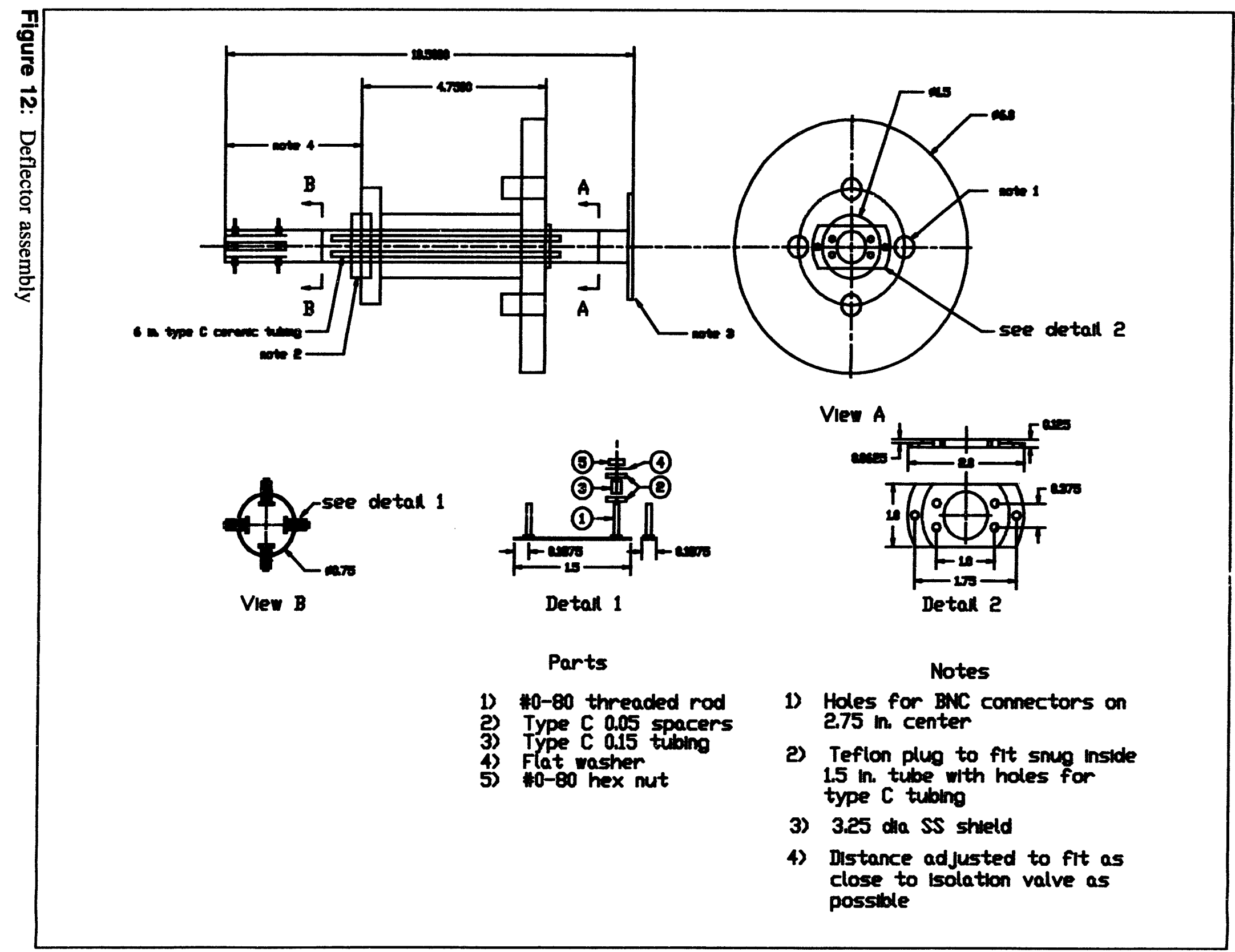


gain, high voltage, and black level. Various contrast enhancements can be made with these controls depending on the image conditions. The output signal from the camera is a standard 1 volt peak-topeak composite video signal which can be interfaced to a wide variety of standard video equipment, including video monitors, video recorders, and frame grabbers.

The Cohu camera was equipped with a standard $35 \mathrm{~mm}$ camera lens with a focal length of 50 $\mathrm{mm}$ and a field stop of $\mathrm{f} / 1.2$. Better performance is provided by this type of lens over the standard $\mathrm{C}$ type lenses associated with video cameras because geometric aberrations have been corrected to provide superior image quality ${ }^{29}$. This arrangement required a $\mathrm{F}$ to $\mathrm{C}$ adapter. The focal length of the lens was changed by installing a $12 \mathrm{~mm}$ extender tube between the lens and the camera. This allowed the MCP to be magnified by the video camera.

The camera was mounted on an optical rail, perpendicular to the main axis of the ion trajectories, approximately $7.5 \mathrm{~cm}$. in front of the MCP. A mirror was mounted at a 45 degree angle to the main axis so that the image could be viewed with the camera. A schematic of the camera arrangement can be seen in Figure 13. Both the camera and mirror were mounted on carriers which could slide along the optical rail. This allowed the camera to be positioned properly and also allowed the mirror to be slid out of the way when taking photographs of the images. A stop was provided for the mirror so that when the mirror was moved out of position it could easily be repositioned in the previous location. The optical rail, mirror and all the mounting hardware for the optics were purchased from Newport Corporation.

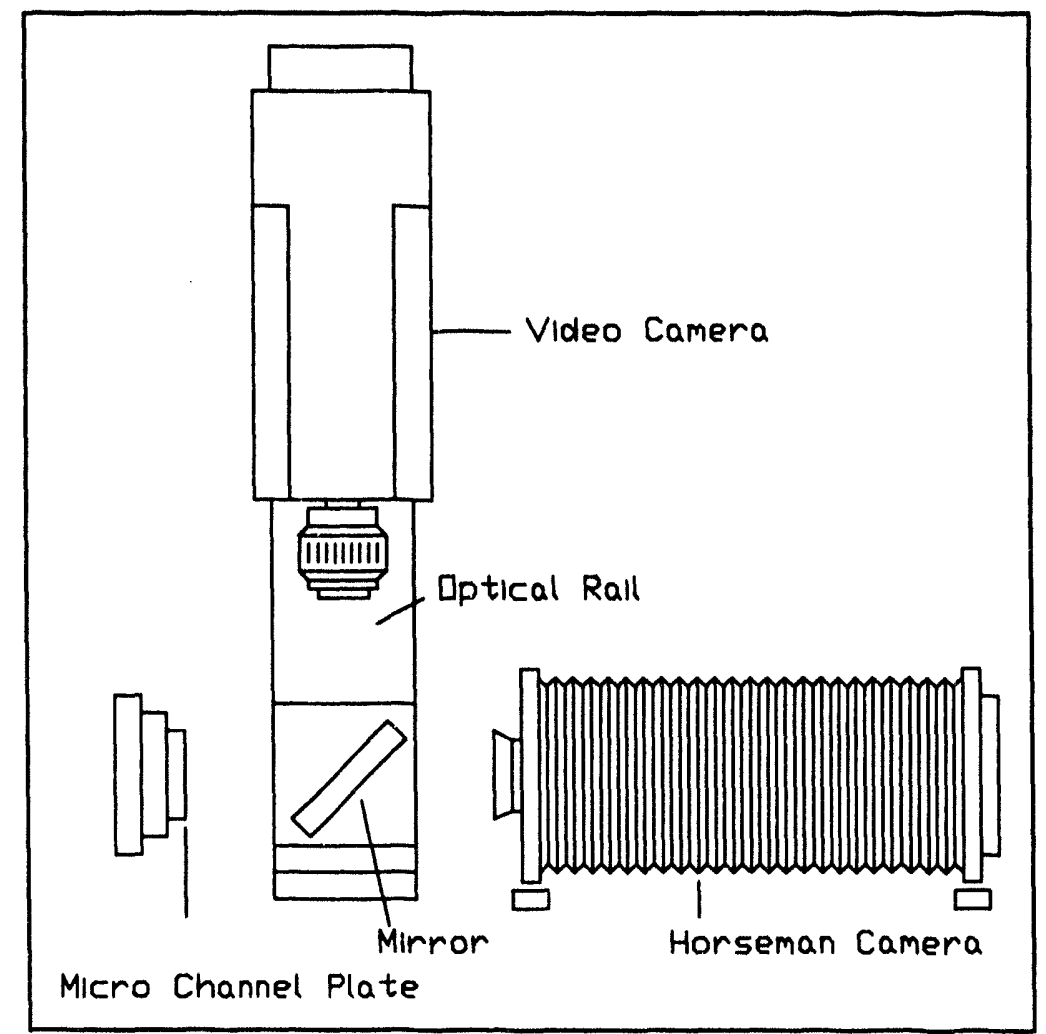

Figure 13: Top view of camera arrangement 


\section{Photographic Camera}

A photographic camera was chosen for recording and archiving images. Cameras provide high resolution and ease of operation. The camera that was used with this instrument was a Horseman Reflex camera. Reflex cameras have a bellows between the front and back planes, allowing the front and back planes of the camera to be positioned in a variety of ways to eliminate optical distortions and control the amount of magnification. 'The magnification is controlled by the distance between the front and the back planes of the camera. If more magnification is necessary, another bellows extension can be installed on the camera. A frosted flass plate located on the back plane of the camera allows the image to be viewed while focusing the zamera.

The camera was equipped with a polaroid film holder allowing $4 \times 5$ polaroid film to used. Polaroid offers a variety of film in both positive, prints only, and positive/negative(P/N), prints, and negatives. However, the $\mathrm{P} / \mathrm{N}$ film is only available in 50 ASA speed which is very slow, requiring long exposure times. The film that we usually use is type 52 positive film, which has a 400 ASA speed rating. Besides polaroid film, $4 \times 5$ negative film can be used. When using this type of film, a special film holder is used and must be loaded in a dark room.

The camera, which is mounted on a rail, was mounted on the table directly in front of the MCP. The rail mount allows the camera to be brought into focus. The camera position relative to the MCP and video camera can be seen in Figure 13.

\section{Data Control System}

A data control system was developed for operating the instrument for two reasons: 1) to make it easier to operate the instrument; and 2) to provide safety features necessary to protect the hardware. While using the data control system, the instrument's focusing and deflector voltages are completely controlled through the computer screen by using the mouse or keyboard inputs. Appendix B is a user's guide that explains the operation and calibration of the instrument. Through the software, provisions were made to store the focusing voltages to data files so that they could be recalled later for image reproduction. Safety features were needed in case of a power supply failure, which could result in too large a voltage differential between focussing elements, causing arcing and possible damage to the power supplies or components inside the vacuum chamber. This safety feature is interactive in the sense that the hardware as well as the software remove the control signals to the power supplies when a failure occurs. The operator is also informed in the event of a power supply failure.

The data control system consisted of a digital-to-analog converter(DAC) board, an IEEE-488 controller board, a signal conditioning circuit, and the computer and software. Both of the computer interface boards were manufactured by National Instruments Inc. A custom design was used for the signal conditioning circuit to provide the appropriate signals along with the desired resolution of control. The software that was written and used for the computer interface was developed using the graphical user interface development environment(GUIDE) ${ }^{30}$ that was developed within the mass spectrometry group at the INEL. 


\section{Hardware}

The DAC board was used to provide the analog control signals for the high voltage power supplies to control the focusing and deflection voltages. Other possibilities for controlling the power supplies were to go with either direct communications such as RS-232 or digital control. Analog control was selected for several reasons: (1) all of the power supplies being used were already capable of being controlled by the use of an analog signal; (2) analog signals are typically faster than direct communications since no communication protocol is necessary; (3) only two signal lines are required for analog control as opposed to digital control which can require up to 16 data lines; and (4) analog signals can easily be generated by a computer with the use of plug-in expansion boards.

The analog output board that was used was the National Instruments AT-AO-10 which is a 10 output 12 bit DAC. The resolution was enhanced by using a signal conditioning circuit. DACs typically are available in 8,12 or 16 bit resolutions. Experiments showed that the most sensitive focusing element, the shield, could effect the images with as little as a 0.2 volt change. In order to achieve this resolution while controlling a $10 \mathrm{kV}$ power supply would require a 16 bit DAC resulting in a resolution of 0.152 volts. Unfortunately, 16 bit DACs generally only come with two outputs. On the other hand a 12 bit DAC can be made to have this kind of resolution by using a signal conditioning circuit that provides a variable span adjustment. This was the method that was chosen and it provided the versatility of being able to manually control the resolutions for each of the power supplies individually. A voltage proportional to the potential on the ion source, which is always at the highest potential, is fed back to the signal conditioning circuit to derive part of the control signal for the other focusing voltages (excluding $\mathrm{f}$, the second to last focusing element). Therefore, any voltage adjustments made to the ion source are tracked by the other high voltage power supplies. Individual focusing voltages are obtained by independently adjusting the output of the power supplies down(offsetting) from the potential on the ion source. The resolutions are enhanced with the variable span adjustments by limiting the maximum offset voltage possible for each of the power supplies.

In addition to increasing the resolution, the signal conditioning circuit provides built in safety features to protect the hardware. Arc protection has been provided in two ways. First, by having the focusing voltages track the voltage on the ion source, the maximum voltage differential between lens elements is restricted. This prevents the operator from inadvertently trying to adjust the focusing voltages through any strange excursions. The second method of protection is provided by using signals from each of the tracking supplies to latch the control signal for the high voltage power supply that floats the filament at high voltage. If any of the tracking supplies fail or are turned off, the hardware removes the signal controlling the high voltage on the ion source. This causes the control signals for the tracking supplies to go to zero. The analog output board also has an 8 bit digital $\mathrm{V} / \mathrm{O}$ port. The software continually monitors the status of the power supplies through this port. In the event of a power supply failure the software control is removed and the operator is informed of an automatic shut down.

The signal conditioning circuit was designed, breadboarded and the circuit board, along with a Lambda LOT-W-5152A power supply, was mounted inside a small metal box. The Lambda power supply was used to both power the signal conditioning circuit and to provide a stable external reference voltage for the DAC board. Signals to and from the computer were made with a 50 conductor ribbon cable. The signals to the power supplies were made with shielded twisted pair cables. The shields were grounded at the signal conditioning end to reduce any induced noises on the control lines. In order to expedite any repairs on the circuit, all of the integrated circuits were 
mounted in sockets. Also, all of the connections to the circuit board were made using an edge connector. A schematic of the signal conditioning circuit can be seen in Figure 14. A complete description of the theory of operation of the signal conditioning circuit can be found in Appendix A.

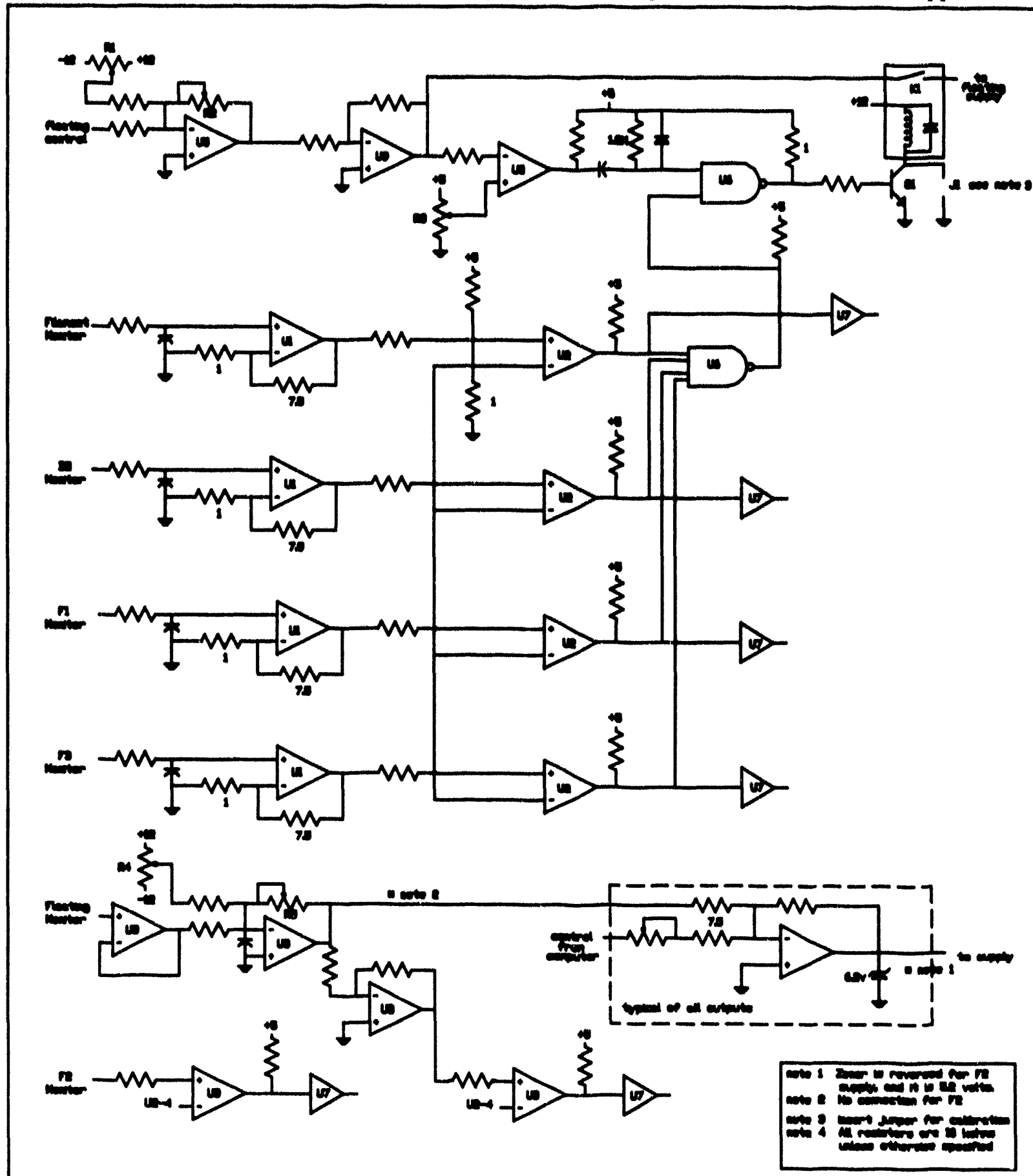

Figure 14: Schematic of signal conditioning circuit

The IEEE-488 controller board( National Instruments GPIB-PCII) was necessary to provide the interface to the Keithley 617 programmable electrometer. IEEE-488, often referred to as GPIB, for 
general purpose interface bus, is a standard hardware and software interface originally designed by Hewlett Packard for controlling their instruments ${ }^{31}$. IEEE-488 has received wide popularity and acceptance due to its ease of implementation and high data transfer rates. Therefore, many programmable instruments utilize the IEEE-488 interface.

\section{Software}

The software for controlling the instrument was developed in the graphical user interface development environment, GUIDE. The program was used to control all of the focusing voltages, the deflectors, and the interfacing to the electrometer. In addition, provisions were made to store voltage settings to files and also to reload settings to reproduce previous images. GUIDE applications are written in $\mathrm{C}$ and provide a variety of controls such as buttons, sliders and display panels. All of the control for the instrument is done using the mouse or keyboard input. Individual help messages for the various buttons and sliders are available by placing the cursor on the artifact and then pressing F1 on the keyboard. GUIDE was chosen as the development environment over commercially available software packages due to its versatility and the ability to adapt to changing demands on the instrument. GUIDE has proven to be very successful for the data system being used on the secondary ion mass spectrometer (SIMS) instrument within the group. The main interface screen that was developed for the imaging instrument can be seen in Figure 15.

The analog output card from National Instruments came with the Ni-DAQ software. Ni-DAQ is the driver-level software that can be called from DOS or from within other application programs. However, when this software was used with the AT-A0-10 board, it slowed down the GUIDE response. Therefore, custom drivers were written in assembler language. The custom drivers improved the response of the interface and were easily implemented from within the program.

The National Instruments GPIB board came with NI-488 MS-DOS software allowing GPIB commands to be called as functions from within application programs written in $C$ and other languages ${ }^{3 !}$. Since the instructions can be called from $C$, it was easy to implement the GPIB interface from within the GUIDE. Along with the general GPIB instructions, instrument specific commands, that select what scale and mode(amps, volts etc..) the instrument is in, are needed to program the electrometer. Complete programming instructions can be found in the instruction manual for the electrometer ${ }^{32}$. The beam current was displayed on a logarithmic bar graph, with ranges from $10^{-13}$ to $10^{-8}$ amps. A numerical display was also used directly above the bar graph (see the lower right corner of Figure 15).

A detailed description of the overall operation of the data control system can be found in the user's manual located in Appendix B. Also, Appendix B describes the procedure for calibrating the hardware and the software.

After the instrument had been constructed, the next phase of the project was to test the instrument to determine any problems and to remedy them to ensure that the entire system was operating properly. The next chapter will describe the methods used to check the operation of the instrument. A discussion of any modifications that were necessary will also be presented. 


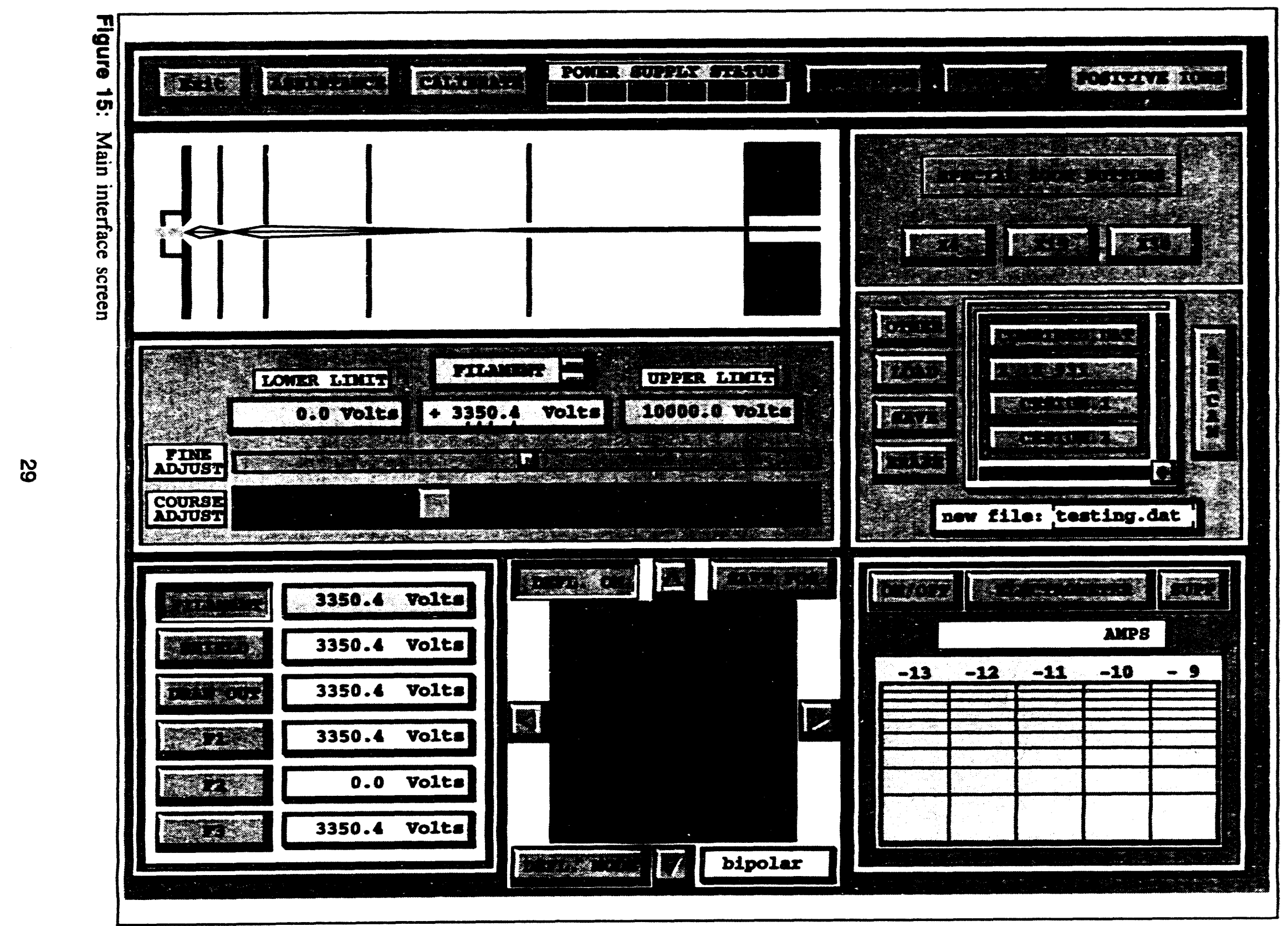


INTENTIONALLY LEFT BLANK 


\section{CHAPTER IV SYSTEM TESTING}

The previous chapters have concentrated on some of the motivations behind the project, on imaging principles, and on the design and construction of the imaging instrument. Before any meaningful ion micrographs could be obtained the system had to be tested. Testing of the instrument was necessary to verify the proper operation of all the components in the imaging system and to make any modifications required to ensure that the instrument was operating satisfactorily. In addition, during the testing of the instrument, the ion optics of the ion lens were modified to optirnize the imaging qualities of the lens.

The testing of the instrument proceeded in five steps: (1) the initial beam testing, including testing the subassemblies such as the MCP, the Faraday cup, and the deflectors with a working ion beam; (2) testing the ion optics to optimize the imaging qualities; (3) testing the data control system; (4) testing the video camera; and (5) evaluating the remote source manipulator. At this point the entire imaging instrument was ready to test.

\section{Initial Beam and Subsystems}

The initial beam test was conducted to verify the operation of the main instrument to confirm that an ion beam could be acquired and imaged. This test was done during a phase of development when the instrument was being controlled manually and proceeded in several steps. The first step was to produce an ion beam which was visible on the MCP. This tested the high voltage power supplies and the MCP. Next the deflectors were tested to see if they could adjust the beam position. In addition, the deflectors were tested to determine the amount of distortion that they introduced into the image. Then the Faraday cup was tested to establish the lower ion current limit of the detector.

The first ion source used in the instrument was a cesium zeolite source. Cesium zeolite was chosen because of its ability to easily produce large ion currents, which simplifies the detection of ions and imaging of the source. This source was not a tube source as described in the system design section, but was a ribbon source in which a small amount of cesium zeolite in aqueous solution was deposited on a rhenium ribbon and dried by running a current through the filament. A scratch was placed across the surface of the cesium zeolite to give a point of orientation while imaging this source. The ion source was installed in a NBS filament hat that mounts directly to the shield plate in the ion lens. Before installing the filament hat, a crude alignment of the source with the ion optics was performed by placing the filament hat on an extra shield plate and then bending the filament to center the source with the aperture in the shield.

It was anticipated that the first ion beam might be hard to detect. Therefore a photometer with a silicon photovoltaic detector was used in conjunction with the MCP. The focusing voltages were initially adjusted to values that had previously been verified in the SIMS instrument. The filament was floated at $5 \mathrm{kV}$ and a current of approximately 2 amps was passed through the filament to heat the source. After some initial trial and error adjustments of the focusing voltages, a beam was detected by the silicon detector and subsequently viewed on the MCP. The following voltages on the various electrodes produced an image on the MCP: 


\begin{tabular}{ll} 
Lens Element & Voltage \\
\cline { 2 - 2 } Filament & 5000 volts \\
Shield & 4989 volts \\
Draw Out & 3800 volts \\
F1 & 4910 volts \\
F2 & 0 volts \\
F3 & 4042 volts
\end{tabular}

The voltages on the MCP were set for 720 volts across the microchannel plate and $3 \mathrm{kV}$ on the phosphor screen. These voltages were kept as low as possible to prolong the life of the detector. The intensity of the image can be adjusted by increasing the voltages but should be limited to $1 \mathrm{kV}$ and $5 \mathrm{kV}$ maximum across the microchannel plate and phosphor screen respectively.

The deflectors were tested by simply adjusting the voltages on the deflector plates. The beam could be moved around on the MCP quite easily. However, distortions in the image were introduced if the beam was deflected any significant amount. Fortunately, the beam was fairly well centered on the MCP screen and did not require deflection during imaging. After the source manipulator had been installed the image could be placed anywhere on the screen with little distortion.

The Faraday cup was tested by inserting the cup into the beam line until it intercepted the ion beam. The ion beam cross section was kept small to ensure that the cup was intercepting the entire ion beam. The discriminator voltage was adjusted for -50 volts to suppress the secondary electrons from escaping the cup. The ion beam intensity was controlled by adjusting the current through the filament. The Faraday cup could detect currents of 0.1 picoamps and the presence of a beam in the 0.01 picoamp range was detectable. However, the signal to noise ratio was high in this range, resulting in unstable readings.

\section{Ion Optics}

Testing of the ion optics was performed to improve the imaging capabilities of the ion lens. The testing of the lens consisted of changing aperture sizes and spacings between lens elements, and observing the quality of the images to determine the effect on the imaging qualities of the lens.

One technique for increasing the spatial resolution in ion optics is to place a small aperture at a crossover point of the ion beam. The small aperture acts as a beam block and eliminates some of the ions that are emitted from the source at high angles and energies and helps to reduce image astigmatism ${ }^{12}$. With this lens, however, the crossover point of the ion beam varies depending on the voltages used to produce the images. Therefore, a small aperture at the crossover would not be practical unless prespecified voltages were used to image the sources. To reduce the ion acceptance angle, the draw out element was moved closer to the ion source, $1.27 \mathrm{~mm}$, and the aperture size was varied between $0.254-2.54 \mathrm{~mm}$. The following observations were made: (1) The smaller the aperture, the better the image quality for higher magnifications. (2) It is much more difficult to obtain images with smaller apertures due to the fact that the voltages had to be adjusted to focus the ions through the smaller aperture. Furthermore, the small aperture tends to shield the effects that fl's(the first lens element after the draw out) potential has on shaping the voltage gradients near the source. Therefore, the shaping of the field gradients near the source must be accomplished by 
adjusting the potentials on the shield and the draw out elements. (3) The small aperture works better for high magnification. As the magnification is reduced, the crossover is pushed further away from the source resulting in the crossover being on the opposite side of the draw out element. Since at low magnifications, ions from the entire surface of the source are being focused through the draw out aperture, simple geometry can show that the size of the aperture places a limit on how far the crossover point can be pushed away from the source.

In addition to the draw out element, the size of the final aperture was adjusted between 0.0508 $\mathrm{mm}$ and $0.508 \mathrm{~mm}$. The smaller apertures help to increase the spatial resolution and depth of field by eliminaiing even more of the ions that are emitted at high angles and energies. The smallest diameter at which an image could still be formed was $0.0508 \mathrm{~mm}$. However, the effort involved in trying to focus the instrument did not warrant using this small of an aperture. Final aperture diameters between $0.1016 \mathrm{~mm}$ and $0.232 \mathrm{~mm}$ were used depending on the features of the source to be imaged.

Other spacings and aperture sizes were tried, but the quality of the images did not improve. The lens design is robust and images were formed with almost any modification to the lens.

SIMION, a simulation package for ion optics, was used to explain the change in the spatial resolution with aperture sizes. SIMION shows that the acceptance angle of the lens for various ion energies is approximately constant for the range of drawout apertures tested when combined with the reduced final aperture. However, with the smaller draw out aperture, the angular and energetic spread of the ions is reduced earlier on in the lens. Thus when the remaining ions are further focused by the last three lens elements the resulting spatial resolution of the image is enhanced because of the fact that the ions forming the image originate at angles closer to parallel to the main axis of the ion lens.

\section{Data Control System}

The data control system was tester to verify that the system could control the voltages without degrading the quality of the images that were obtainable under manual control. Before the data control system could be used to image any sources, the system had to be setup and calibrated. By following the procedure outlined in Appendix B, the instrument was setup as follows:

\begin{tabular}{|c|c|c|c|}
\hline Element & $\frac{\text { Minimum }}{\text { Voltage }}$ & $\frac{\text { Maximum }}{\text { Voltage }}$ & Resolu \\
\hline Filament & 0 & 10,000 & 2.44 \\
\hline Shield ${ }^{*}$ & $x \cdot 1,000$ & $\mathrm{x}$ & 0.24 \\
\hline Draw Out & $x-5,000$ & $x$ & 1.22 \\
\hline $\mathrm{F} 1^{\circ}$ & $x-5,000$ & $x$ & 1.22 \\
\hline $\mathrm{F} 2$ & 0 & 5,000 & 1.22 \\
\hline $\mathrm{F} 3^{\circ}$ & $x-5,000$ & $\mathrm{x}$ & 1.22 \\
\hline
\end{tabular}

* - minimum voltage no less than approximately 800 volts

$x=$ voltage on the ion source(FILAMENT)

The maximum offset voltages from the voltage on the source were limited to $5 \mathrm{kV}$ because the 
electrical feedthroughs are only rated for $5 \mathrm{kV}$ post to post. While operating the instrument in the manual mode, arcing had been observed when the voltages between posts exceeded the $5 \mathrm{kV}$ rating.

The deflectors were set up to operate in the bipolar mode and to have a maximum voltage of 60 volts on any one plate. The maximum voltage was kept to a minimum in order to give better control over the positioning of the beam. For purposes of deflecting the ion beam off the MCP, the software is permanently setup to provide a 500 volt differential between two of the plates. By having a large voltage differential, deflection of the beam off the screen is assured under any of the accelerating voltages applied to the ion lens.

\section{Data control testing}

After calibrating the instrument, the entire system was tested under computer control. A cesium zeolite tube source was imaged. The initial images were unstable and appeared to have noise on one or more of the focusing elements. In order to help filter any unwanted noise that might be induced on the control lines, $10 \mu \mathrm{F}$ capacitors were placed across the control inputs to each of the power supplies. These filter capacitors helped but did not cure the instability of the images. Upon further investigation, it was determined that the Spellman power supplies have a $10 \mathrm{~K} \Omega$ resistor between the common on the control input and earth ground. The added resistance was causing a ground loop in the control signal lines. By placing a $220 \mu \mathrm{F}$ capacitor between the control common and earth ground, the noise problem was eliminated. Since all of the control commions of the Spellman supplies are in parallel, only one capacitor was necessary to filter the unwanted noise.

\section{Video System}

The video system needed testing in order to ascertain the usefulness of being able to remotely view the images while operating the instrument. The testing consisted of acquiring an image by observing the video monitor and then switching to the photographic mode to see if the images needed to be tweaked to obtain a good photograph. After the video camera was properly focused, it was not necessary to further focus the image when switching between video and taking photographs of the image. The video camera system proved to be quite valuable in that it was more sensitive (usable image with a faceplate illumination of $0.0002 \mathrm{Lux}^{28}$ ) than the human eye, thus allowing faint images to be observed on the video monitor. Also, the image was magnified on the monitor, thus aiding in the focusing of the instrument.

\section{Ion Source Manipulator}

The remote manipulator for the ion source was tested to determine if any vibrations had been introduced which degraded the images. In addition, the value of being able to reposition the ion source relative to the rest of the ion lens needed to be evaluated. The test consisted of acquiring an image of a cesium zeolite tube source and then taking photographs of the image to examine the images for any sign of vibrations. The photographs were enlarged and the small spots on the photographs were blurred. This blurring was not visible on the portions of the photograph not corresponding to the ion image, thus indicating a vibrational problem. The vibrations could be felt on the vacuum chamber and appeared to be coming from the roughing pump. The pump was then placed on a piece of foam on the floor and the final connection between the roughing pump and the turbo pump was made with a piece of rubber hose. This did not totally eliminate the vibrations on the vacuum chamber. Next an 
end plate which was built to dampen vibrations was installed in the ion gun. The end plate slides over the ceramic rods of the ion lens assembly and the springs maintain pressure on the remote source mount. This couples the remote assembly to the ion lens and reduces any out of phase vibrational modes between the two. The last action taken was to put a support jack under one end of the mounting rail for the photographic camera. Upon taking more photographs and having them enlarged the blurring effect observed earlier was eliminated and the resulting resolutions were greatly increased.

The value of being able to reposition the ion source is two-fold. The first benefit is that the source can be moved around, allowing various portions of the ion emitting source to be viewed on the MCP. The second advantage is that it is not always possible to adjust the focusing voltages just right to sharpen certain features in an image. The manipulator can be used to move the ion source in and out relative to the shield of the ion lens and bring those features into focus, or to improve the overall focusing of the ion image.

Once the instrument's operation had been verified the next step was to acquire experimental data. The next chapter in the thesis will describe the procedures used to accumulate the data and will present the results of the experiments. Light and ion micrographs will be contrasted to demonstrate the capabilities of the ion imaging instrument. 
INTENTIONALLY LEFT BLANK 


\section{CHAPTER V \\ EXPERIMENTAL RESULTS}

The final stage in the development of the imaging instrument was to obtain experimental data to confirm the proper performance of the instrument. The data was evaluated to determine some of the operating characteristics such as minimum detection limit and spatial resolution. The experiments also demonstrated the capability of imaging both cations and anions. The ultimate test of the instrument is the quality of the ion micrographs that are obtainable.

In this chapter are described the experimental procedures and results used to evaluate the instrument's abilities and representative results. The first section will describe the method used to determine the lower detection limit of the instrument. The second section will present the procedure for determining the spatial resolution. This section will contrast the ion micrographs with light micrographs of three different sources, demonstrating the instrument's ability to image cations and anions.

\section{Minimum Detection Limit}

Many factors contribute to the minimum ion current level that the instrument can image. Since the ions are being converted to electrons and then to scintillations in the MCP, the gain and accelerating voltages placed on the electrons will have a significant influence on the minimum detection limit. The ion micrographs are recorded on film for storage purposes. The speed of the film and the exposure time will therefore contribute to the minimum limit. The lens being used on the photographic camera has a minimum field stop of $f / 5.6$. A lens with a smaller $f$-stop could be used to enhance the lower limit of the photographic capabilities of the instrument. However, a larger aperture would result in the loss of some of the fine details in the photographs. Also, if the image can not be visually evaluated for focusing purposes either by directly viewing on the MCP or with the video camera, then the images may not reveal any useful information. The sensitivity of the video camera could also be used to reduce the lower detection limit of the instrument. However, to take full advantage of a more sensitive video camera, we would require other recording media such as a video recorder or frame grabber.

\section{Procedure}

A simple procedure was used to determine the lower detection limit. A small diameter ion beam was obtained and viewed on the MCP. Then the ion current was reduced to the point where the images could no longer be photographed reliably. The experiment to determine the minimum detection level was performed only once. Therefore the accuracy and any statistical information regarding the actual detection limit were not obtained. The results were used only as a relative performance index to give an indicator of the types of ion sources that might be able to be imaged in the instrument. If more accuracy is necessary, the experiment could be performed several times on different occasions. The results could then be analyzed to arrive at a more reliable value for the minimum detection limit. 


\section{Results}

In order to determine the lower detection limit of the instrument, a cesium zeolite tube source was used. The cesium source was again chosen because of the ease of ion production in the cesium zeolite sources. The beam current could be readily controlled by varying the current in the filament, which in turn controls the temperature of the ion source. The baseline suppression capability of the Keithley electrometer was used to suppress a baseline of approximately $3 \times 10^{-13}$ amps, resulting in a background reading of $10^{-16}$ to $10^{-15}$ amps. A small bearn cross section was used to ensure that the entire ion beam was being intercepted by the Faraday cup. The following voltages were used to produce a $4 \mathrm{~mm}$ spot size as observed on the MCP.

\begin{tabular}{ll} 
Lens Elument & Voltage \\
\cline { 2 - 2 } Filament & 6464 volts \\
Shield & 6349 volts \\
Draw Out & 6300 volts \\
F1 & 6296 volts \\
F2 & 0 volts \\
F3 & 5157 volts
\end{tabular}

With a filament current of 2 amps the resulting beam current that was measured was $1.4 \times 10^{-}$

${ }^{13}$ amps and could easily be seen on the video system. Then the filament current was reduced to 1.8 amps which resulted in a beam current reading of $1.5 \times 10^{-14}$ amps. Although the reading was unstable it was obvious that an ion beam was present at this level. With the micro channel plate voltage set at 800 volts and the phosphor voltage set at $4.02 \mathrm{kV}$, the image could still be seen on the video system. A photograph was taken with 400 ASA polaroid film and required a 5 minute exposure time with the aperture set at $\mathrm{f} / 5.6$, completely open. The filament current was then reduced to 1.7 amps. At this point the image could not be seen using the video system. The voltages on the MCP were increased to 950 volts and $4.5 \mathrm{kV}$ and a photograph was taken using 3000 ASA polaroid film. The photograph required 20 minutes to expose with the field stop set at $f / 5.6$. The resulting photograph just barely reveals the image of the source. Longer exposures could possibly be used to improve the ion micrograph, but with the added possibility of vibrations degrading the image. Therefore, $1.5 \times 10^{-14}$ amps was used as the lower limit on the practical detection limit. Using this value for the beam current, the ion flux density was calculated as follows:

$$
\begin{gathered}
\frac{1.5 \times 10^{-14} \mathrm{amps}}{1.602 \times 10^{-19}}=9.3632 \times 10^{4} \frac{\mathrm{ions}}{\mathrm{sec}} \\
\text { flux }=\frac{9.3632 \times 10^{4} \mathrm{ions}}{\pi \times(2 \mathrm{~mm})^{2} \mathrm{sec}}=7.4548 \times 10^{3} \frac{\mathrm{ions}}{\mathrm{mm}^{2} \mathrm{sec}}
\end{gathered}
$$

Extending this value of the flux density to an image that would just take up the entire active area of the MCP $\left(13.79 \mathrm{~cm}^{2}\right)$, the ion current should be $1.646 \times 10^{-12}$ amps to achieve a usable image. For higher magnifications, where not all of the ions are striking the MCP, higher beam currents would be 
necessary to produce good images. In reality this value is probably on the low side since the small spot size image did not have a very uniform intensity (the edges were much brighter than the center). However, it illustrates the relative minimum detection limit of the instrument.

\section{Spatial resolution}

The spatial resolution of an imaging instrument can be defined as the smallest discernable feature in a given image. The spatial resolution is often reported as line pairs $/ \mathrm{mm}$ indicating how close two lines can be and still be distinguished on an image. Several factors can limit the overall spatial resolution obtainable by the instrument. These are: (1) The ion optics used to form the images in the system. (2) The diameter and spacings of the channels in the MCP detector. The microchannel plate used in this instrument has a maximum theoretical spatial resolution of $40 \mathrm{LP} / \mathrm{mm}$. This results in a resolution of $25 \mu \mathrm{m}$ as viewed directly on the face of the MCP. (3) The quality of film being used to photograph the images will also effect the resolution of the instrument. Several types of film have been used to record the ion micrographs. Polaroid type $\mathbf{5 2}$ has been used to obtain positive prints and has a resolution of $22 \mathrm{LP} / \mathrm{mm}$. Another Polaroid film that has been used to obtain both positive and negatives is type 55. The negatives have a high resolution of $155 \mathrm{LP} / \mathrm{mm}$. In addition to Polaroid film, Kodak T Max 100 and 400 film has also been used, with maximum resolutions of $200 \mathrm{LP} / \mathrm{mm}$ and $125 \mathrm{LP} / \mathrm{mm}$ respectively.

\section{Procedure for determining resolution}

For this instrument the spatial resolution was approximated by: 1) Measuring a feature in a light micrograph of the ion source that has a known magnification of $64 x$. 2) Measuring the corresponding feature in the ion micrograph to obtain an estimate of the magnification factor in the ion micrograph. 3) Selecting and measuring the smallest feature that could be readily distinguished from the rest of the image and then using the magnification to calculate the estimated spatial resolution of the instrument. Two sources were used to determine the spatial resolution. A third source was imaged and the ion micrographs are included since they reveal interesting topographical information about the source.

\section{Results}

The first source was a cesium zeolite tube source that had been used in earlier experiments. The cesium tube source was not only used to measure the spatial resolution, but was also used to demonstrate the operability of the instrument in the cation mode. After the source had been operated for a while it appeared to shrink in the tube and some light lines began to show up in the ion images. These lines appear to be hairline cracks in the surface of the emitter and are visible in both the light and ion micrographs. The light micrograph and two ion micrographs can be seen in Figure 16. Using the highlighted portion to estimate the magnification to be $145 \mathrm{x}$ the spatial resolution was estimated to be approximately $3.8 \mu \mathrm{m}$.

This cesium zeolite source has been imaged on many occasions and demonstrates the ease of repeatability of the images. Ion micrographs with similar quality and magnifications have been obtained upon demand. When the same focusing voltages were used, usually only minor adjustments were necessary to reproduce the images. However, the voltages used for the images have not always been the same. This can be attributed to factors such as a variance in the temperature of the source or 


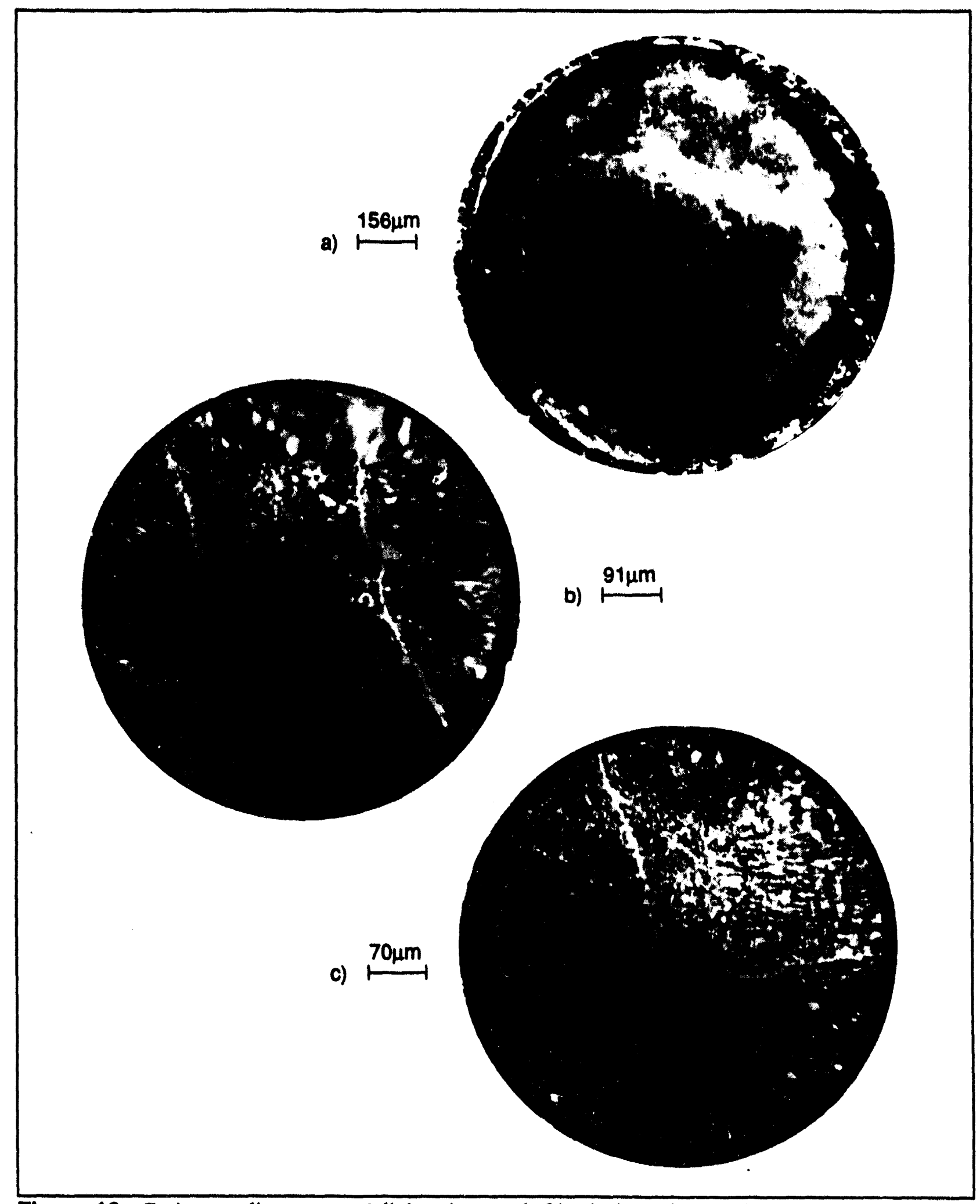

Figure 16: Cesium zeolite source: a) light micrograph $64 \mathrm{x}$ b) ion micrograph 110x c) ion micrograph $145 \mathrm{x}$ 
the actual position of the source relative to the rest of the ion lens. The variations in the voltages were not a great concem and affirm the robustness of the ion optics ability to image with many different voltage settings.

The second ion source that was used to estimate the spatial resolution of the instrument was a perrhenate $\left(\mathrm{ReO}_{4}\right)$ anion source. This was a tube source that had previously been used in the SIMS instrument. Refer to Figure 17 for the light micrograph and ion micrograph. The highlighted portion was used to estimate the magnification to be approximately $160 \mathrm{x}$ in the ion micrograph. The corresponding spatial resolution is $3.1 \mu \mathrm{m}$ which is in good agreement with the cesium zeolite source. Of course, this magnification and resulting spatial resolution will depend on the ion source being imaged and the focusing voltages that are used when producing the image. The dark highlighted region in the ion micrograph was further analyzed with scanning electron microscopy(SEM) to see if there were any chemical differences between this region and the neighboring bright spots.

Unfortunately SEM did not reveal any noticeable differences. The dark region may just be a topographical formation on the surface of the ion source.

A third ion source that was imaged was another perthenate tube source that had been used in the SIMS instrument. A cross pattern has been etched in the surface using a hypodermic needle. The images are interesting because they reveal topographical information about the source, as shown in Figure 18. The shadowing effect that reveals the topography can possibly be attributed to the emission of ions from the surface following a cosine function. The majority of the ions being emitted from a surface leave at an angle normal to the surface. As the angle from the normal increases the number of ions decreases. Thus, the majority of the ions emitted from surfaces that are not perpendicular to the main axis of the ion lens are not focused onto the MCP for viewing. This creates the shadows corresponding to the topography in the surface of the ion emitter.

All of the ion micrographs presented have maximum magnification factors of $145 \mathrm{x}-160 \mathrm{x}$. When the magnification was increased the field of view in the image became smaller until the image was no longer visible. This limit is the result of the combination of the ion lens and the MCP detector. A more sensitive MCP might be able to extend the magnification further. Also, the magnification could be increased by enlarging the photographs. However, the magnification would be limited by the resolution of either the MCP or the film being used.

The photographic camera magnifies the image on the MCP by approximately 1.25 . In the ion micrographs reported in this thesis an additional enlargement has been given to make the ion micrographs the same size as the light micrographs. The overall magnification from the photographic procedures is approximately 1.5 . If $150 \mathrm{x}$ is taken as a representative maximum magnification on the ion micrographs, the maximum magnification on the MCP screen would be $100 \mathrm{x}$. Based on the MCP limit of $40 \mathrm{LP} / \mathrm{mm}$ the maximum theoretical resolution limited by the MCP would be approximately $0.25 \mu \mathrm{m}$. The film with the lowest resolution that was tested was $22 \mathrm{LP} / \mathrm{mm}$ and the resulting maximum theoretical resolution would be approximately $0.36 \mu \mathrm{m}$ on the ion micrograph itself. By analyzing the ion micrographs the limiting factor at this stage in development of the imaging instrument is probably the ion optics used to image the ion sources. Fortunately, the resolutions being obtained are more than adequate for the investigations that are planned in the near future. 


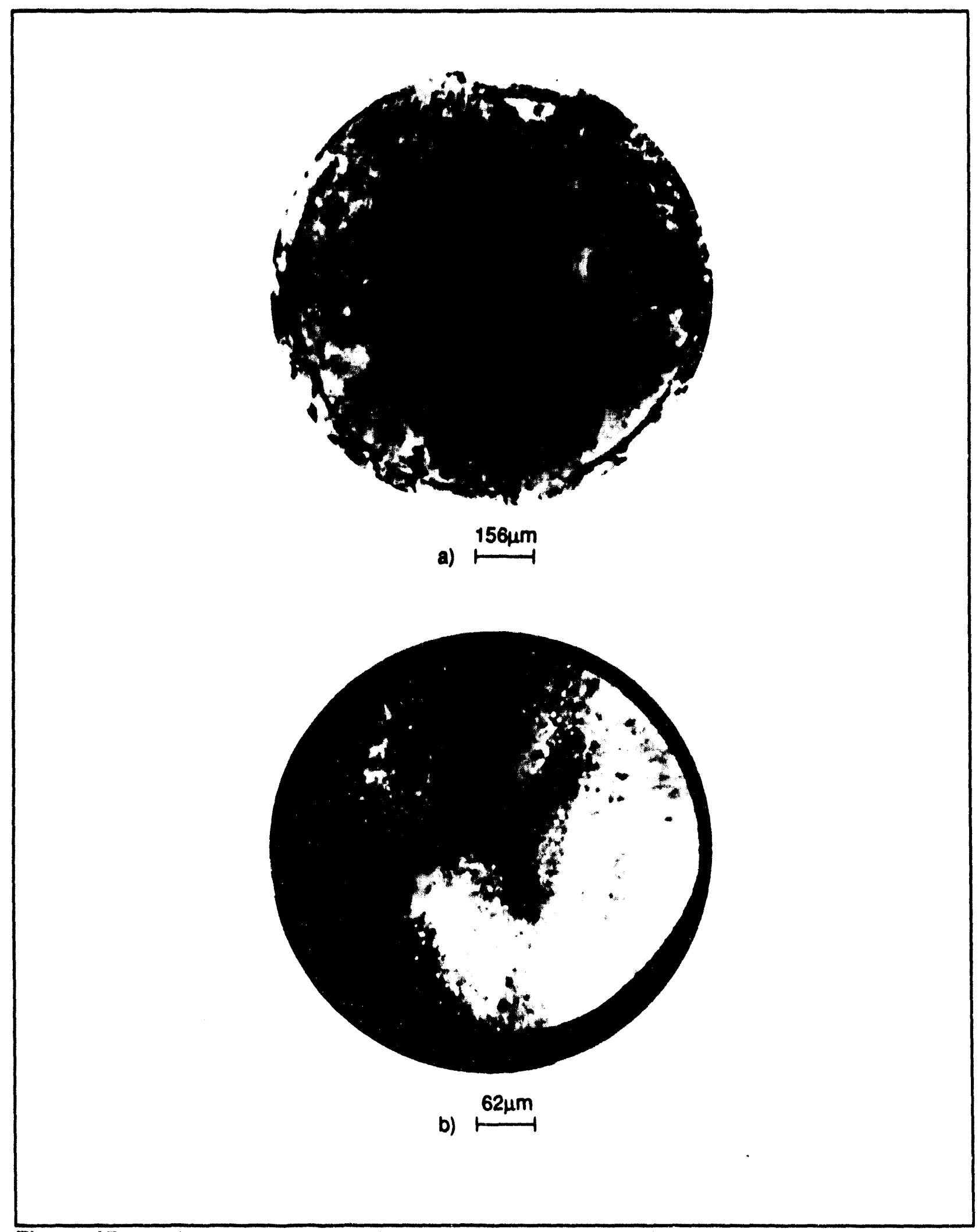

Figure 17: Perrhenate ion source a) light micrograph $64 \times$ b) ion micrograph $160 x$ 


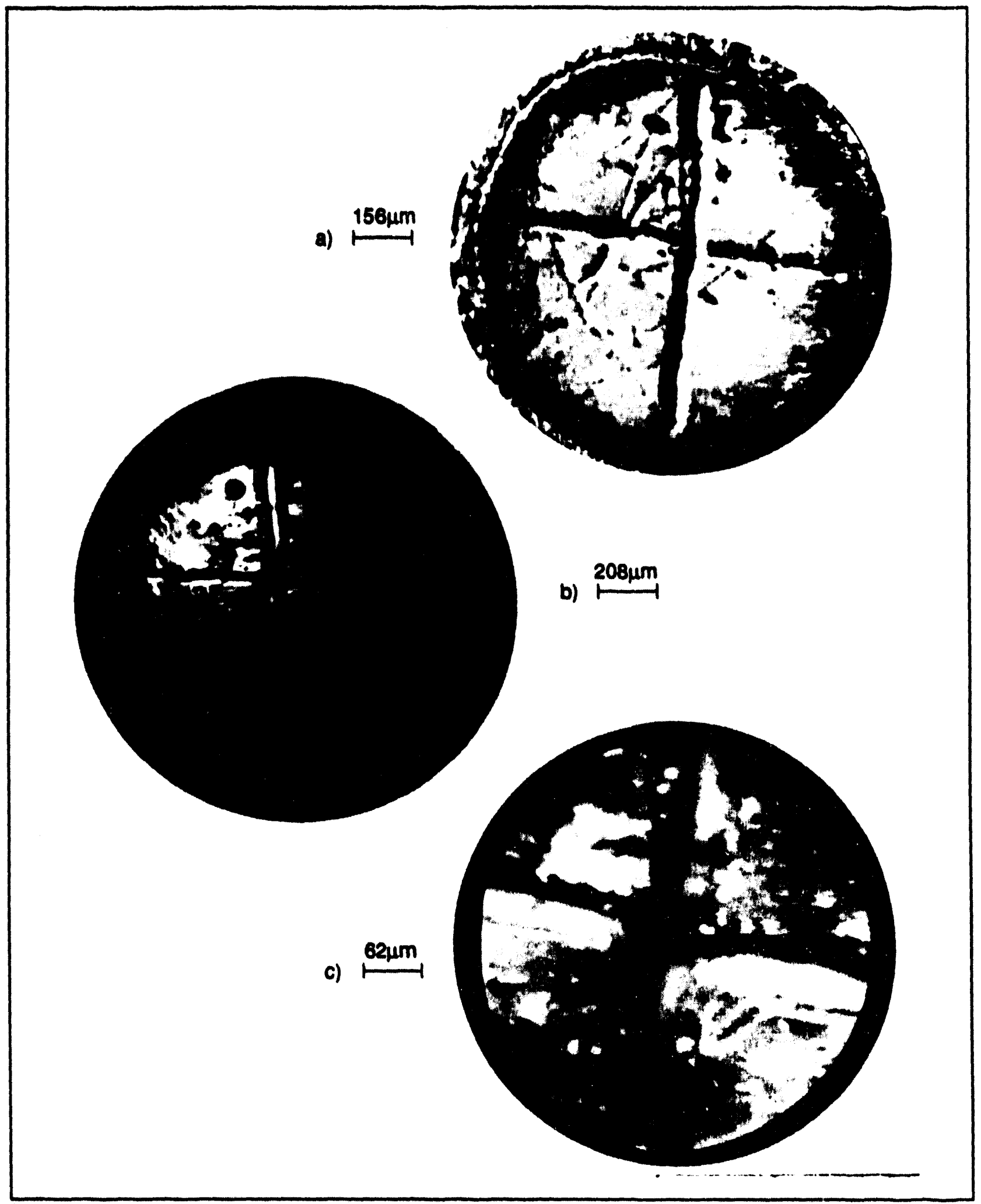

Flgure 18: Perrhenate ion source $\# 2$ a) light micrograph $64 x$ b) ion micrograph $48 x$ c) ion micrograph 160x 
INTENTIONALLY LEFT BLANK 


\section{CHAPTER VI \\ CONCLUSIONS}

The primary goal of this thesis was to construct and demonstrate the operability of an instrument for imaging the ions being emitted from ion sources used for surface analysis. The results show that the instrument can image both cations and anions with a spatial resolution down to approximately $3-4 \mu \mathrm{m}$. Work that needs to be done includes imaging other ion sources and fine tuning the performance of the instrument. A dual (Chevron) microchannel plate detector could be irstalled on the instrument to improve the lower detection limit. However, this may result in a loss of spatial resolution sue to the space charge effects on the electrons between the two microchannel plates. The video system has proven to be invaluable to the operation of the instrument. A time lapse video recorder could be interfaced to the system allowing sources that are varying with time to be imaged and recorded or to do life time studies on ion emitters. It may also be useful to install a frame grabber on the system which would allow more sophisticated image processing to be done on the images.

The information presented in this thesis affirms the ability of the imaging instrument to provide direct images of the ion current densities of the ions being emitted from the sources. Care must be taken while interpreting these images since focusing effects can lead to faulty conclusions. For example a dark region in an image could be the result of ions not being emitted from that area, as in the case of a chemical dissimilarity at that point, or it could be just a topographical formation on the surface, such as a divot. The limited depth of field requires that the focusing voltages be adjusted to try to bring the dark regions into focus (possibly resulting in the dark regions becoming brighter) to ensure that no ions are originating from these areas. This can also be done by physically moving the ion source closer to, or further away from, the rear plane of the ion lens with the source manipulator. By carefully interpreting the ion micrographs obtained from the instrument, the images can be a road map indicating areas of high and low ion emission that need further examination with other instruments, such as SEM, to develop a better understanding of the ion emission processes.

Two different ion emitting systems have been studied in this thesis, cesium and perrhenate ion emitters. Preliminary results in both systems indicate that the majority of the ions are being emitted from the surface of the material and not from the interface between the material and the substrate. This was one of the first questions that was hoped to be answered with this instrument. Another ion source that has been studied since the data that was presented in this thesis was taken is bismuth borosilicate. The imaging instrument showed that the ion emitting surface was in liquid form. The imaging instrument gave the first indications that the glass had undergone a phase transformation. Based on these results, it is believed that the imaging instrument will be a useful analytical tool for studying the spatial origin of the ions from various ion emitters. The results of these investigations are expected to help in the understanding and possible production of new ion sources for use in surface analysis. 
INTFNTIONALLY LEFT BLANK 


\section{APPENDIX A \\ SIGNAL CONDITIONING CIRCUIT THEORY OF OPERATION}

When providing control signals to various devices, in this case power supplies, it is necessary that the signals match the requirements of the controlled devices. Zero and span circuits can often be used to provide signal conditioning when the output from the computer does not match the requirements of the supply. A signal conditioning circuit for the imaging instrument was necessary to zero and span the control signals. It also provided a safety interlock to protect the power supplies and other hardware from damage due to arcing. The interlock consisted of a tracking circuit that made the high voltage power supply that floats the ion source the master, and the supplies for the shield, draw out, F1, and F3 the slaves that track the voltage adjustments made to the source. This was done to ensure that the voltages between focusing elements did not become too large and create arcing. The second feature of the safety interlock was a feedback system in which a proportional voltage from each of the slave supplies was used to latch the control signal to the master supply. If any of the supplies fails or is shut off then the control signal to the master supply will be removed and thus effectively shut down the remaining power supplies. A third feature of the interlock is a digital feedback signal to the computer. The status of the power supplies is fed back to the computer and if a critical power supply has failed then the software will initiate an automatic shutdown and warn the operator of a failure. A schematic of the signal conditioning circuit can be seen in Figure 19. In this Appendix we will describe the theory of operation of this signal conditioning circuit.

The control circuitry for the master supply can be seen in Figure 20. The control voltage for the master supply is first passed through an inverting zero-span circuit, where a gain of approximately 1.2 is used to boost the 5 volt(full scale) signal to 6 volts. The zero adjustment was not originally in the design, but after powering up the instrument it was determined that the power supplies could not be pulled all the way down to zero volts. The signal is then passed through an inverting amplifier with a gain of unity to get the signal in proper polarity for programming the power supplies.

As the control signal for the source is increased, it is compared to a threshold setting on the comparator U3. When the level exceeds the threshold, the output of U3 goes low. The resistor capacitor network acts as a "one shot" allowing a short duration, approximately 0.21 second, low pulse to be applied to one input of U6. The low pulse on the input to U6 causes the output of U6 to go high and bias on Q1. While Q1 is biased on, the relay $\mathrm{K} 1$ is energized and the control signal for the master supply is routed through the normally open contacts to the power supply. The low pulse is the trigger for the hardware interlock. Before explaining the latching mechanism of the interlock, it is necessary to describe the method by which the other power supplies develop their control signals.

After the master supply receives its control signal, a voltage proportional to the output voltage is present at the voltage monitor output of the supply, 1 volt $=10 \mathrm{kV}$. The monitored signal is fed back to the signal conditioning circuit, where it is amplified and zero corrected (see Figure 21). The signal is amplified to give approximately the same amplitude as the signal being fed to the filament supply. Then this signal, along with the control for the individual supplies, is combined in an inverting summer circuit to produce the control voltages for the other supplies. Consequently, the power supplies track the high voltage adjustments made to the ion source. In Figure 21 a typical output for the control circuit is shown. 


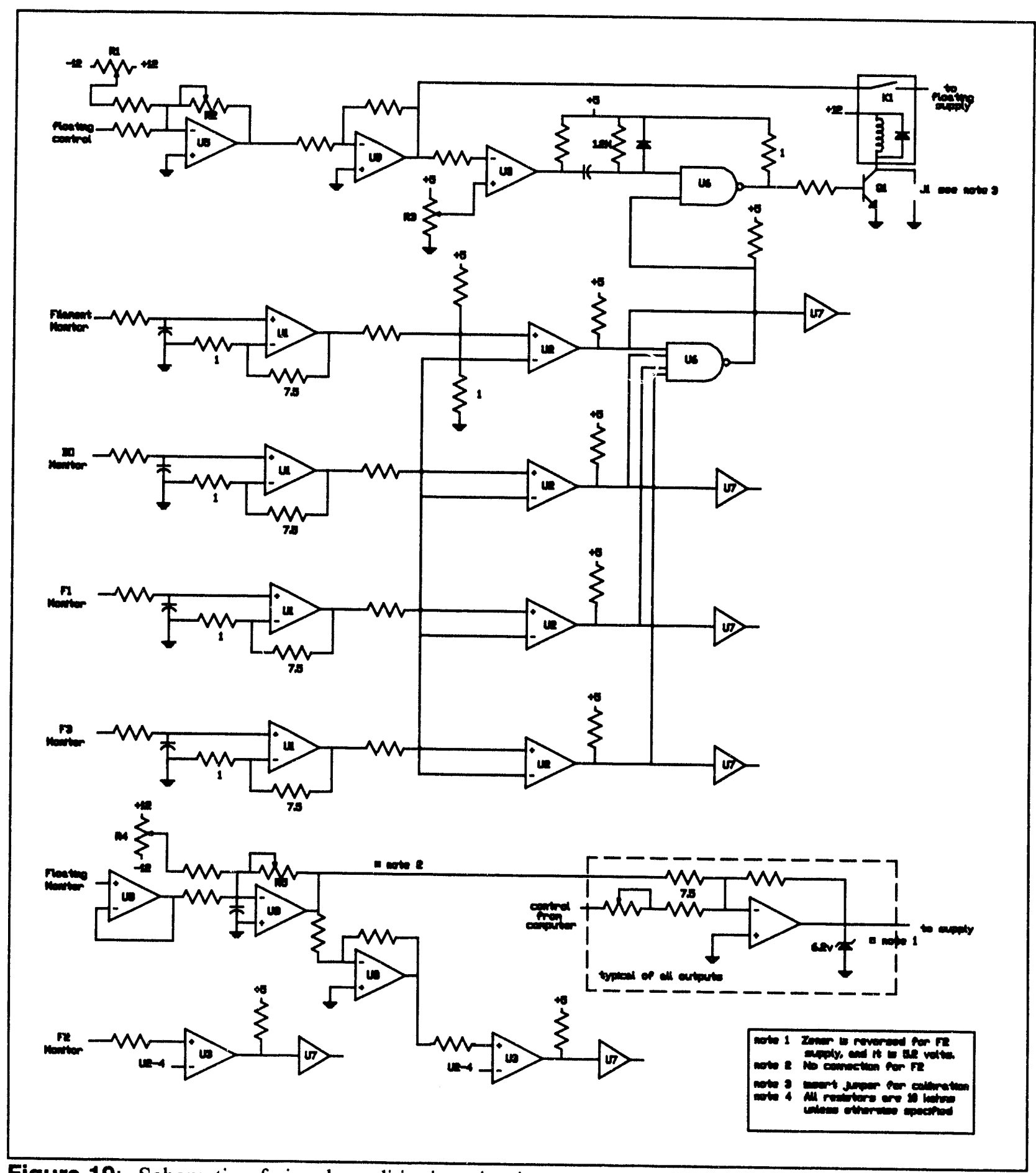

Figure 19: Schematic of signal conditioning circuit

The control signals are subtracted from the voltage developed from the master supply. In order to increase the resolution, a gain adjustment is provided to adjust the full scale span of each supply. For instance, the shield supply gain is adjusted to provide a $1 \mathrm{kV}$ maximum offset from the source, resulting in a resolution of 0.24 volts (out of 10,000 volts). 


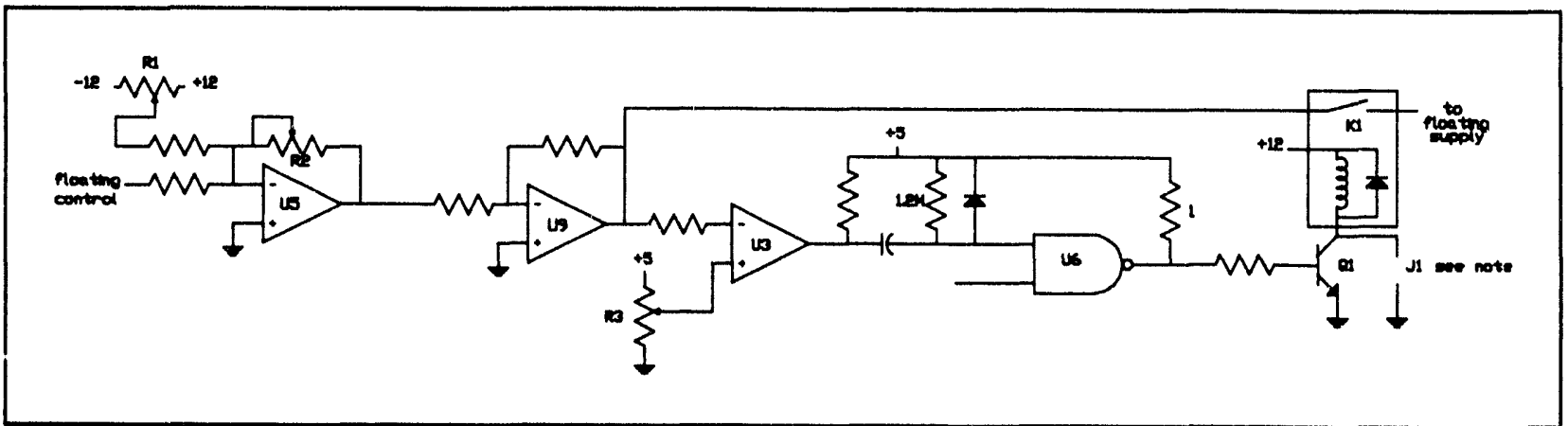

Figure 20: Control circuitry for high voltage supply which floats the filament supply

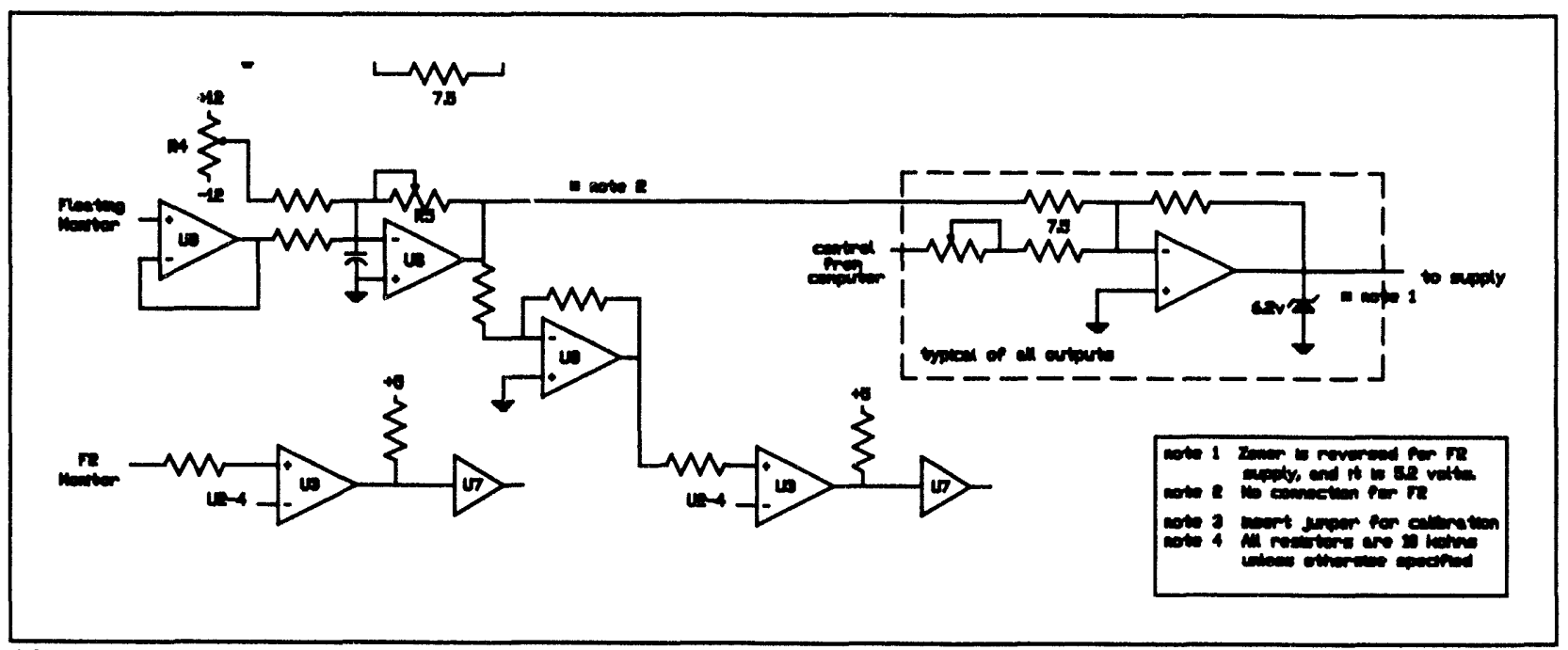

Figure 21: Output circuitry

During initial adjustments of the focusing voltages, the master supply is adjusted first. After the trigger pulse is received on the base of Q1, the master supply voltage increases, developing the corresponding control signal for the other supplies. As the outputs of the other supplies begin to increase, their monitor outputs are fed back into the conditioning circuit (see Figure 22) where they are amplified and compared to a threshold setting of approximately 0.45 volts. As long as all the voltages remain above this threshold, then the transistor will remain biased on after the trigger pulse returns high. Subsequent failures or power down of one or more of the supplies will unlatch the interlock by turning the transistor off, removing the control signal from the master supply. Once control to the master supply is removed, the outputs of the other supplies automatically go to zero by virtue of the inverting nature of the control output circuitry.

The digital feedback of the power supply status is accomplished by sampling the monitored feedback signals used to latch the interlock and then passing them through U7, refer to Figure 22, a data buffer driver circuit. The other two power supplies are also monitored by similar circuits (refer to Figure 19). The software continually monitors the status of the power supplies and in the event of a failure will shut down all high voltage supplies. 


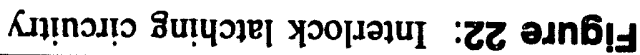

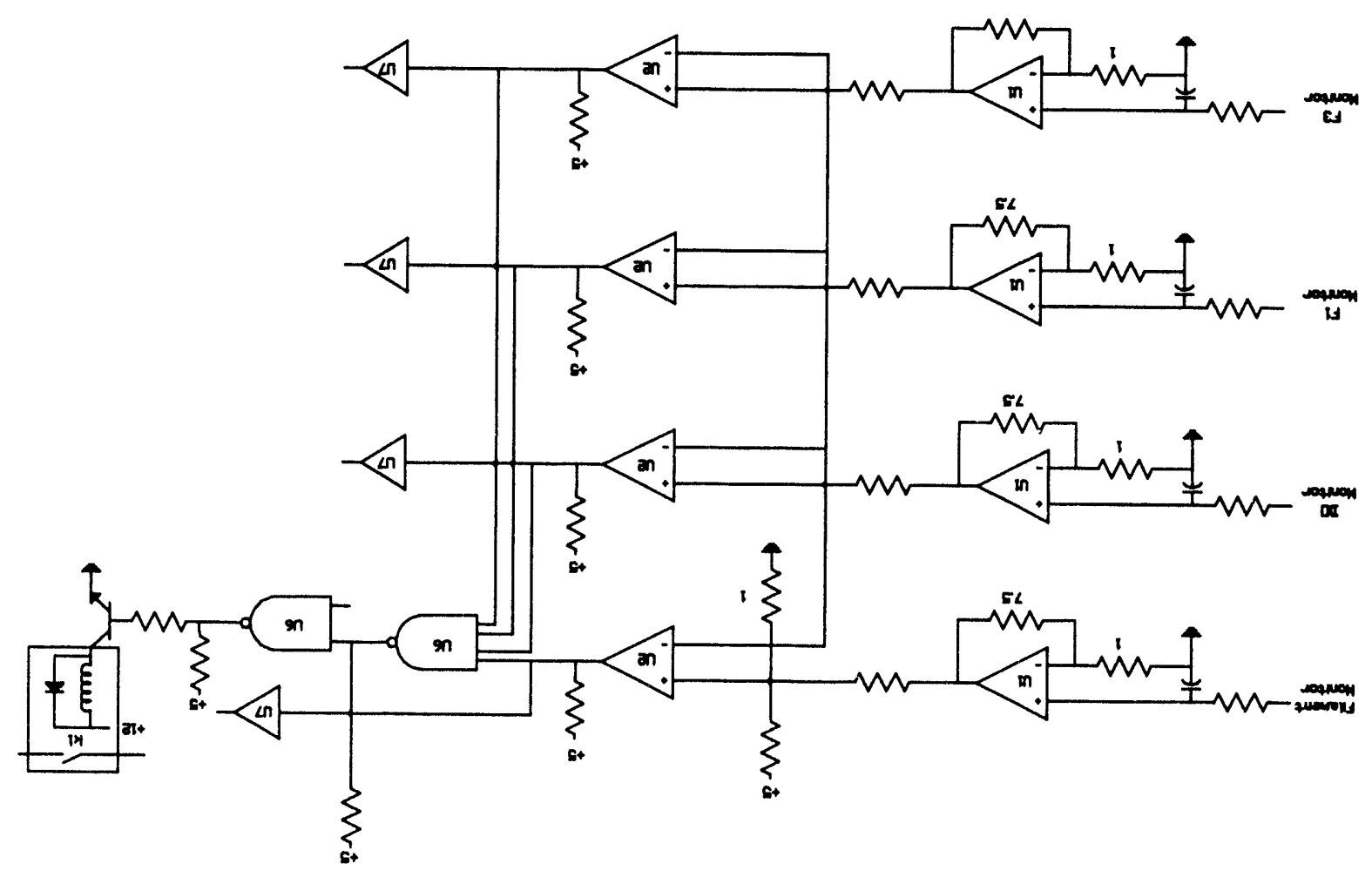




\section{APPENDIX B \\ USER'S GUIDE FOR THE IMAGING INSTRUMENT}

\section{Introduction}

The ion self imaging (ISI) instrument has been designed to be easy to use and with a few precautions should allow the most novice of operators to image ion sources without damaging the instrument. A data control system has been integrated into the system. The control system is used to control all of the focusing voltages as well as the deflectors for positioning the ion beam on the microchannel plate screen. The user interface has been developed using the graphics user interface development environment (GUIDE). The interface provides a variety of buttons, sliders, etc..., which are activated by using either the mouse or keyboard to control the system. If you are familiar with WALK-ABOUT or other GUIDE applications, operating this instrument will be a snap. Specific help is available on the various controls by pointing to an object and then pressing the $\langle\mathrm{F} 1\rangle$ key or holding down the shift key and clicking any mouse button. Focusing voltages can be stored in data files and later recalled to reproduce previous images.

In addition to the software, a signal conditioning circuit has been used to provide zero and span adjustments to enhance the resolution of the control signals. It also provides a safety interlock that protects the power supplies and other hardware from damage due to arcing. The interlock consists of a tracking circuit that makes the power supply that floats the ion source at high voltage, the master and the supplies for the shield, draw out, F1, and F3 the slaves that track the voltage adjustments made to the source. This feature allows the total voltage to be adjusted without major defocusing of the lens, since all voltages go up or down proportionally. Individual focusing voltages are generated by adjusting the outputs of the power supplies down from the voltage on the master. This is done to ensure that the voltages between focusing elements do not become too large and create arcing. The second feature of the safety interlock is a feedback system in which a proportional voltage from each of the slave supplies is used to latch the control signal to the master supply. If any of the supplies fails or is shut off then the control signal to the master supply will be removed shutting down the remaining power supplies. A third feature of the interlock is a digital feedback signal to the computer. The status of the power supplies is fed back to the computer and if a critical power supply has failed then the software will initiate an automatic shutdown and warn the operator of a failure. A schematic of the signal conditioning circuit along with the various pin outs on the connectors can be found in the last section of this guide.

The hardware, as well as the software, needs to be setup and calibrated to operate the system; see the calibration section. If an ion source needs to be installed in the instrument, proceed to the next section on how to vent the system. If an ion source is already in the instrument the section on powering up the instrument will get you started.

\section{Vacuum System - Venting and Pump-Down Procedures}

The source chamber is held under vacuum by a turbo pump which allows for quick turn around times between source changes. The first step in using the instrument is to install an ion source. This proceeds in three steps: 1) The source chamber must be vented. 2) The ion source must be installed on the ion gun assembly. 3) The source chamber needs to be evacuated. The following 
section will discuss the procedures used to vent the source chamber and then re-evacuating the chamber. Also, the procedure for venting and evacuating the flight tube portion of the chamber will be explained, however, this portion of the instrument rarely needs to be vented.

\section{Source chamber}

The ion source chamber is normally isolated from the flight tube portion of the instrument during times of non-use. Before venting the ion source chamber turn off the ion gauge, verify that the isolation valve is closed, and that the power to the high voltage power supplies is off. Now turn off the turbo pump and the roughing pump. Next remove any bolts that are securing the hinged access flange with the viewport on the rear of the source chamber. The chamber is designed to be vented through a dry nitrogen line attached to the turbo pump. There are two valves that must be opened to allow the nitrogen to be introduced into the source chamber. The first valve is near the regulator just above the roughing pump. Make sure that the pressure on the regulator is adjusted for only a few psi and then open this valve. The other valve located on the top of the turbo pump can now be opened. You should hear the turbo pump winding down at this point. After the pressure inside the source chamber rises, the access door can be pulled back and laid down, allowing access to the ion lens and source assembly.

To evacuate the source chamber, first close the valves controlling the dry nitrogen. Then the access door can be pressed closed and while holding slight pressure on the access door turn on the roughing pump. This will suck the hinged flange tight against the back of the source chamber and you can let go of the flange. Replace the bolts that were securing the hinged flange to the source chamber. After the pressure as seen on the thermocouple gauge begins to decrease, the turbo pump can be turned on. Finally the ion gauge can be powered on. It normally takes approximately $30-60$ minutes for the pressure to pull down to the mid to low $10^{-7}$ Torr range at which point the instrument can be operated.

\section{Flight tube}

When venting the flight tube of the instrument make sure that the isolation valve for the ion pump is closed, and that the power to the microchannel plate and high voltage power supplies is off. If the source chamber is to be vented, follow the procedure described above before venting the system.

The flight tube needs to be roughed out by the use of the combination of the roughing and turbo pumps. It is usually fastest if the ion lens has been removed from source chamber. Otherwise, the conductance is limited by the final aperture of the ion lens. After removing the ion lens, open the isolation valve to the flight tube. Then follow the procedure for evacuating the source chamber. The isolation valve for the ion pump should not be opened until the pressure in the flight tube is in the $10^{-6}$ Torr range. After the ion pump is pumping on this portion of the instrument, the source chamber can be isolated, vented, and the ion lens can be reinstalled in the system.

\section{Lens Installation and Removal}

Normally the ion lens does not need to be removed from the instrument. However, on occasion it is desirable to modify the ion optics (ie. change the size of one or more of the lens apertures) to improve the imaging. After venting the system, remove the wires on the ion lens. Next, 
remove the spring plate assembly by removing the four screws in the back of the ceramic posts. The spring plate will now slide off of the ion lens. After removing the spring plate, remove the rectangular support bracket which holds the ion source mount. Then adjust the remote mounting assembly upward to get the assembly out of the way for removal of the ion lens. The ion lens should be able to be pulled straight back toward you and out of the instrument. Installation of the lens is the reverse procedure.

\section{Ion Source Installation and Removal}

In order to install an ion source in the instrument, make sure the high voltage has been turned off and then follow the procedure for venting the source chamber. If an old source is in the instrument remove the wires attached to the ion source. Next, the two long hex nuts that secure the source hat (small cylindrical mount) can be removed. Be careful not to drop the two washers that are used for spacers on the threaded posts. The source hat can now be removed from the instrument. A small alignment jig has been constructed of Kimbal Physics components. Place the source hat on this jig and secure it with the same two hex nuts that are used to hold the hat in the instrument. Place the ion source in the source hat (be careful not to damage the ion source) and secure it in place with the two set screws accessed from the sides of the hat. The ion source can then be bent to align it with the aperture in the front of the alignment jig. Remove the source hat from the jig and install it in the instrument. Place the wires on the filament posts of the ion source and then evacuate the source chamber.

\section{Operation}

Once an ion source has been installed in the instrument, then the instrument can be turned on. First access the interface software by typing "image" at the DOS prompt. The interface screen should come up on the computer screen(see Figure 23). If you are unfamiliar with the operation of the computer interface, it may be useful to operate the interface with the signal conditioning circuit and power supplies powered off. The interface will appear to be functioning properly just as if you were operating the instrument. Try exploring the various controls by holding down the shift key and moving the mouse around and clicking the mouse button on the controls to access the specific help messages. For an explanation of the operation of the instrument's controls skip over the power up procedure section and read the rest of the operation section.

\section{Power up procedure}

The instrument needs to be powered up in a certain order to ensure that everything between the computer, the signal conditioning circuit and the high voltage power supplies is in the proper state. The following order should be used:

1) The firmware on the DAC board is setup to apply half of the control voltage to the system, therefore a program has been written to zero the outputs. This program executes from within the autoexec.bat file and consequently the computer must be turned on first. Certain programs are initiated when the computer is first turned on such as loading a device driver to allow the software to communicate with the GPIB board, and the video board is set up for $800 \times 600$ resolution. 


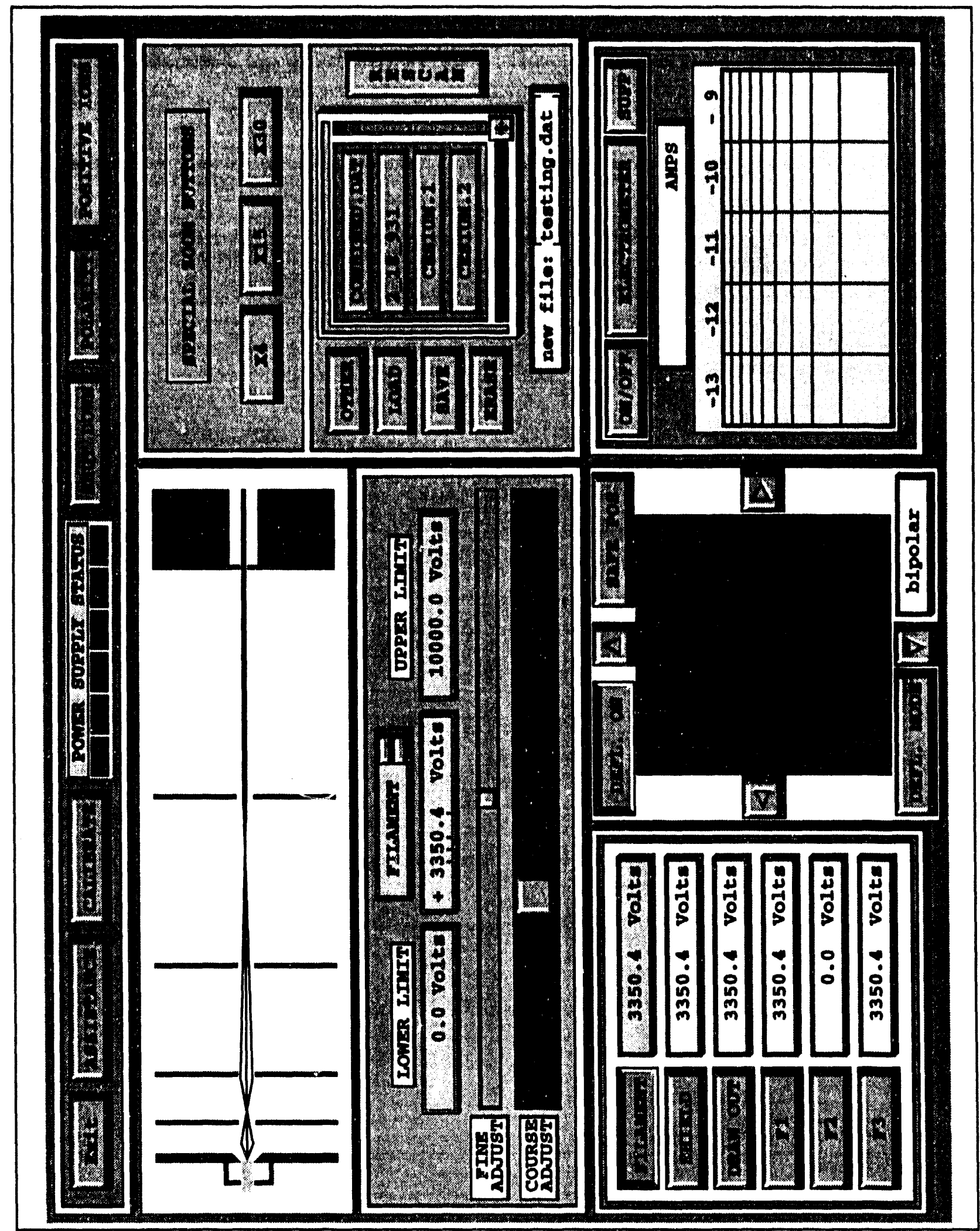

Figure 23: Main interface screen 
2) After the computer is turned on, turn on the signal conditioning circuit, by turning the switch located on the blue and white metal box to the on position. The red light indicates that the circuit is tumed on.

3) Finally the power to the high voltage power supplies can be turned on by opening the equipment cabinet and turning on the power strip. This will apply power to the power supplies which control the focus, deflectors, the discriminator voltage on the Faraday cup and the MCP. The MCP is a dual "Chevron" type of detector and the voltage across the two microchannel plates should be kept as low as possible ( 2000 volts max) and the voltage on the phosphor screen should be below 5000 volts.

4) The video camera and monitors can now be turned on. Do not remove the lens cap on the video camera prior to applying power to the camera-this can result in damage to the image tube.

5) The electrometer should be turned on and allowed to warm up if any critical current readings are going to be taken.

After powering up the instrument and accessing the interface software by typing "image" at the DOS prompt, the main interface screen should be visible on the computer screen. The majority of the control of the instrument is accomplished from within this screen. If current measurements are going to be performed, place the electrometer in the remote mode by clicking the mouse button on the on/off button located on the electrometer display(lower right corner of Figure 23). A baseline reading will be visible on the logarithmic display. Allow the reading to stabilize and then press the SUPP button to suppress this reading from the electrometer. The instrument is now ready to be operated. Open the isolation valve between the source chamber and the flight tube. The filament current which heats the ion source must be operated manually. Slowly increase the filament current to the desired setting, a meter shows the approximate current being supplied to the filament. Now the high voltages can be applied to the ion lens elements. The following section describes how the interface works to generate the voltages for focusing the ion images, and also some of the other features available through the interface. 


\section{Solection and adjustment of focuaing voltages}

The first step in adjusting the high voltage power supplies is to select the supply labeled FILAMENT. Due to the interlock built into the hardware and software, this supply must be selected first. This is the supply that floats the filament and source at high voltage. Power supply selection is accomplished in one of two ways. The first method of selection is to push one of the selection buttons corresponding to the power supply, see Figure 24. The second method of selection, is to use the selector located in the upper center of the slider surface, see Figure 25. By clicking the mouse buttons, the lens element supplies can be selected one at a time. After a power supply is selected, the panel located next to the button changes color and the label for the element appears in the panel located in the center of the slider control surface, see Figure 25. In addition to displaying the element name, the corresponding minimum and maximum element voltages are displayed and the main control slider will turn red and green and move to the position corresponding to the current setting for the element. A schematic drawing of the ion lens is shown directly above the slider surface, see Figure 26. As the power supplies are selected, the element that is being adjusted will be drawn in bright red.

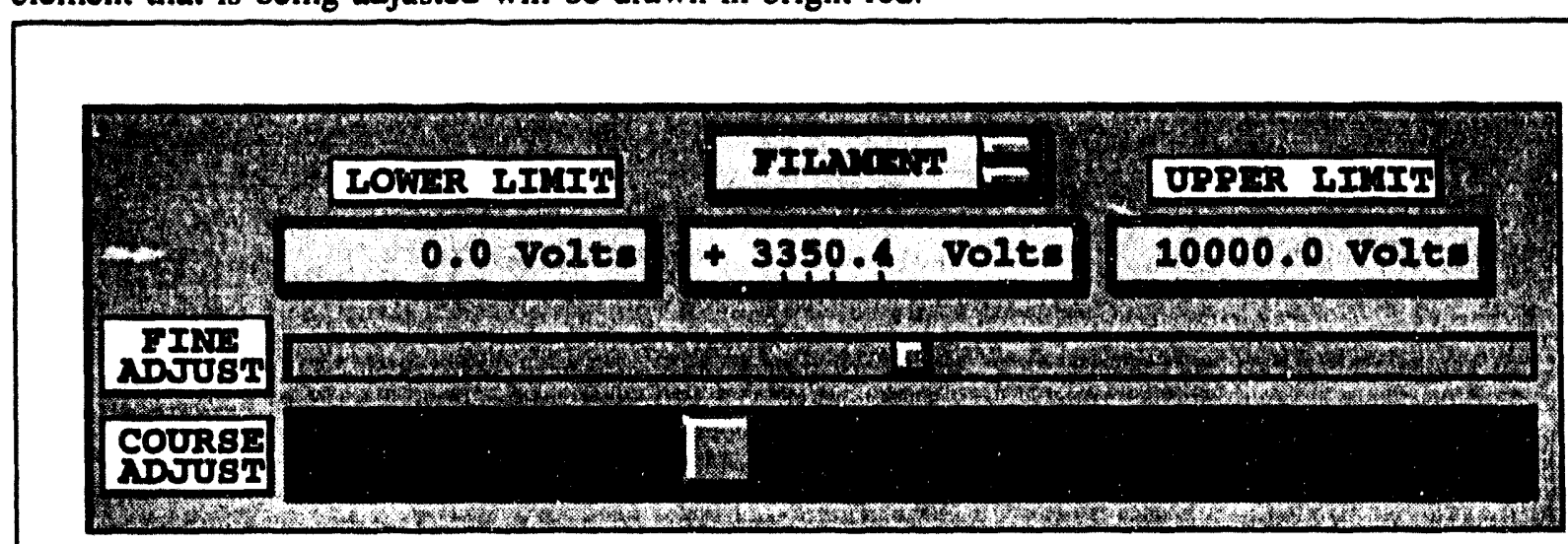

Figure 25: Main control sliders

The various lens element power supplies are all controlled from the two sliders located in the slider surface shown in Figure 25. One of the sliders is for fine adjustments and the other is for course adjustments. The smallest increment discernable on the fine adjustment is 1 out of 4095 where as the course adjustment is approximately 10 out of 4095 . Also the fine adjustment slider rolls around, that is when it reaches one end of its travel it will move to the opposite end of the slider and keep moving. This movement is similar to the action of a ten turn potentiometer. The course adjustment has a velocity damping factor built in to the movement. If the slider, which is controlled 


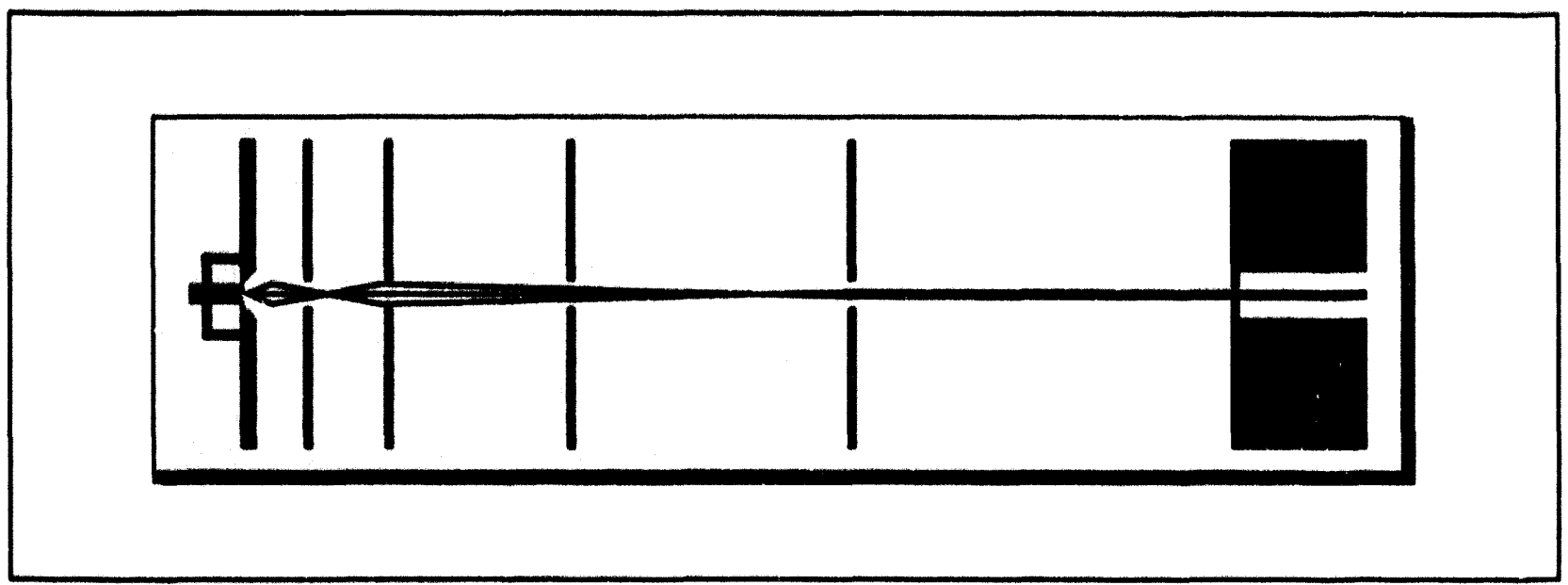

Flgure 26: Lens schematic

by the mouse, is commanded to move to fast, then the velocity damping kicks in and slows the slider down. In this way, the rate the voltage can be changed is limited.

The voltage can also be adjusted by using the center display panel, located above the fine adjust slider. The upper limit of the adjustment is limited to the hundredths position so that large voltage adjustments are avoided. By positioning the cursor over one of the digit place holders, indicated with a small tick mark, and then clicking either the left or right mouse button the voltage can be adjusted up or down. Numerical values for these digits can also be entered via the keyboard.

As the voltages are adjusted, the approximate output voltage is shown in the display panel located directly above the fine adjust slider and also on the corresponding display located just to the right of the selection button. Since the signal conditioning circuit is set up so that the power supplies corresponding to the Shield, Draw Out, $\mathrm{Fl}$ and F3 all track the Filament supply, when the filament supply is adjusted so are these other supplies and the corresponding output values are displayed in their displays.

Now select the FILAMENT supply and adjust the course slider adjustment. Watch the meters on the high voltage supplies and notice that all of the power supplies except F2 track the adjustments made with this slider. Select one of the other power supplies such as the SHIELD and make adjustments to the voltage. Notice that this voltage adjustment causes that particular power supply output to offset from the setting on the FILAMENT. This is how the individual focusing voltages are adjusted.

\section{Saving and loading focusing voltages}

Located above the electrometer is a file management window; see Figure 27. From within this area you can store the current settings of the power supplies. To store the data, a file must first be created by moving the cursor to the new file window and typing the name of the file. A binary file will be created in the directory image. The first byte in the file is a length of file indicator which is used to keep track of how many sets of data have been stored in a particular file. After creating a file, it will show up as a new button in the center window. Select this file by clicking the mouse on the desired button. After selecting a file, click on the save button. A message will appear that asks 
whether to replace, append or cancel. The append feature lets the user collect multiple sets of data to be analyzed later and used to update the information for the special zoom buttons. If you want this file to contain only the current lens element settings, select replace.

In order to erase files, you first select a file from within the button window. Then select erase and the program will give a prompt to make sure you want to erase the selected file. If you want to then answer yes.

The interface also lets you load a previously stored set of element settings. Select the file which contains the settings to be loaded. At this point the program looks at the length of file indicator, which is updated every time you store data in one of the files. If this value does not match the actual length of the file the program assumes that the file is not compatible with this application and warns the user. The option is given to convert the given file to a compatible form. If you are not sure if this is a data file then do not convert the file because it could destroy what was in the original file.

\section{Special zoom buttons}

Three special zoom buttons have been created to help in finding the ion beam. Due to varying parameters such as ion source position, these buttons do not always result in an image. However, the settings are usually fairly close and by tweaking some of the voltages will generally result in finding the ion beam. The three buttons are currently setup to provide settings that result in images with approximately $4 x, 15 x$, and $30 x$ magnifications. These buttons can be changed dynamically while using the instrument. By collecting a series of focusing voltages and saving the data using the append feature of the save, the data can then be used to generate new focusing parameters for the buttons. Regressional analysis is applied using the voltage on the iun source as the independent variable to generate the settings for the other power supplies. The titles for these buttons can also be changed during an imaging session. All of this information is stored to a file and recalled each time the instrument is operated.

In order to update the button's information, first collect some data and store it in a file. Then select this file and use the "OTHER" button on the file handle window to access another screen. From within this screen the data can be plotted by using the plot, the data can be sent to a printer, or the buttons can be updated by selecting update buttons. After selecting update buttons another window will appear and from this window you can select what degree of fit, either linear or $2^{\text {nd }}$ order, and which button to use. The current settings will appear along with the regressional coefficients proposed for each of the power supplies. If you want to see how close the actual fit is, hit cancel to go back to the previous screen and then select plot. From the plot screen you can graph the various lens element voltages that were recorded along with the proposed regressional curves that can be used to generate 
the focusing voltages. If you want to update the information then return to the update buttons screen and update the coefficients for the particular button.

\section{Electromoter}

The electrometer is interfaced to the system through a general purpose interface bus (GPIB) otherwise known as IEEE-488. A controller board is installed in the computer and a cable must be attached to the electrometer. A discriminator voltage is applied to the discriminator plate on the Faraday cup, and when the electrometer is first turned on, a baseline reading usually results. The Keithley electrometer has built into it a baseline suppression capability. To null the baseline, place the electrometer in the remote control mode of operation by clicking the mouse button on the ON/OFF button on the electrometer display (refer to Figure 28). If the electrometer does not respond, as in the event of having the electrometer off or the cable unplugged, then the software will inform you of an error and tell you what to check to remedy the problem. Allow the reading on the electrometer to stabilize and

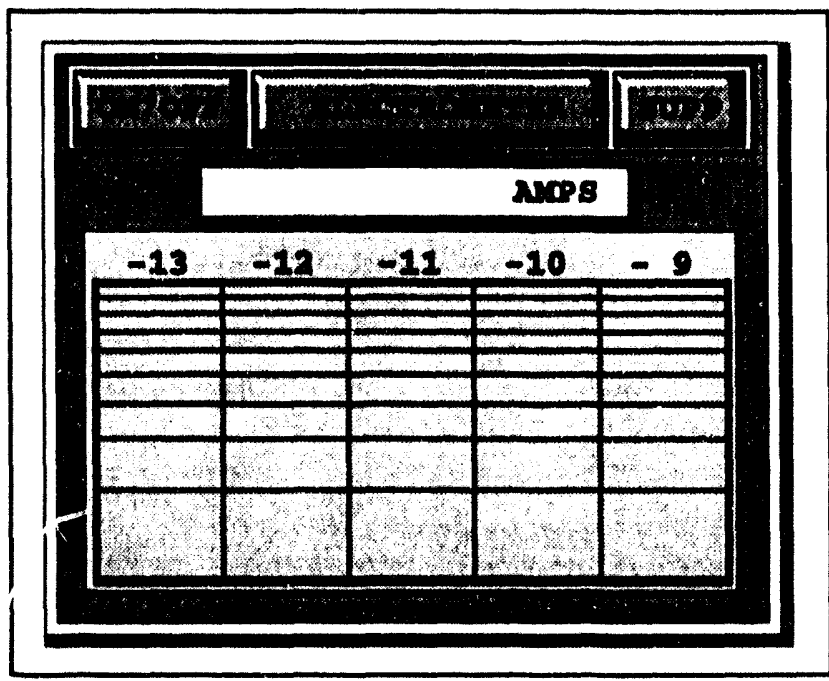

Figure 28: Electrometer display then press the SUPP button to suppress the baseline reading. Now the electrometer should be ready to take any current readings that are necessary. The main software is set up to split the service between reading the electrometer and mouse movements that are used for the rest of the interface. The mouse movements have priority. However, the movements may appear to be jerky. Therefore, when the electrometer is not being used the interface display for the electrometer should be turned off by clicking on the ON/OFF button.

\section{Deflectors}

The deflectors are operated from the screen by using a two dimensional slider(refer to Figure 29). The screen is setup to look like a crosshairs pattern corresponding to the front of the MCP screen. By moving the button in an intuitive manner, the image can be easily repositioned around on the face of the MCP. The deflectors can also be controlled from the four arrows corresponding to the four primary directions. By clicking the right mouse button on one of the buttons the deflectors will move continuously, while clicking the left button will increment the deflectors only one position. When the cursor is positioned over the deflector display, the arrow keys on the keyboard will also move the deflectors. The deflectors must first be activated by turning the deflectors on with the ON/OFF button on the deflector window. When the deflectors are off all of the voltages on the deflector plates are removed. The ion beam can be deflected off of the screen by clicking on the SAFE POS button. The display for the deflectors will display a message "Beam in safe position". In order to reactivate the deflectors and get the image back on the screen, you can click the mouse button on the safe position button again, or move the cursor over the deflector window and click the mouse button or hit return on the keyboard. 


\section{Polarity reversal}

The polarity of the power supplies must be manually switched. Remove power from the power supplies and then switch the polarity in the following manner. The Spellman power supplies require that a square plate be removed from the back of the power supply chassis and then rotated 90 degrees(as indicated on the plate) and reinstalled in the power supply. The Bertan supply only needs a screw to be rotated in the back of the power supply. Once the polarity of the power supplies match the polarity of the ions you want to image, the software can be set up to display the proper polarity of the voltages. Click the mouse button on the POLARITY button on top of the main interface screen. A new window will appear over the file support and special zoom buttoris(refer to Figure 30). The polarities can be selected individually or they can all be inverted simultaneously by selecting change ion polarity. If the filament polarity changes, so will the display showing what type of ions are being imaged.

\section{Shutting down the system}

The procedure for shutting down the instrument is basically the reverse of the power up procedure. Slowly reduce the filament current to zero. Then you can exit the interface program by pressing the EXIT button. This will automatically remove the control voltages applied to the power supplies. Also, at that point you are given the option to save the current polarity and calibration data to a file. If you have not changed any of the calibration data then click on the cancel button and the program will terminate and return to the DOS prompt. If you want to save the settings, answer yes to save the data to a file and the program will terminate and return to the DOS prompt. If you do not want to exit the program, but instead just want to turn off the power supplies and signal conditioning circuit, use the SHUT DOWN button on the top of the main screen. This button will adjust the power supplies to zero volts but will not exit from the program.

After the high voltage power supplies are at zero volts, then turn the power off to the supplies by opening the cabinet and turning off the power strip. Before turning off the signal conditioning circuit wait a minute or so to allow the filter capacitors to discharge. Then you can turn off the power 
to the signal conditioning circuit. Isolate the source chamber from the flight tube, to eliminate the possibility of the instrument venting through the mechanical pumps. Before turning off the power to the video camera put the lens cap on.

\section{Setup and Calibration Procedure}

The data control system which was used for the imaging instrument consists of the interface cards that plug into the AT compatible personal computer, the signal conditioning circuit, and the interface software. During imaging procedures the entire instrument is controlled through the computer except the ion source manipulator, the filament current, and the cameras. The hardware and software were designed to give an interlock protection for the hardware which will disable the control signals in the event of one or more of the power supplies either being powered down or failing. Before the imaging system can be operated for the first time, the computer and the hardware need to be configured properly to have the interface display the proper voltage levels and to set the safety interlock threshold.

\section{Computer Setup}

Applications developed using the graphics user interface development environment(GUIDE) can support several video modes including EGA, VGA, and SVGA with up to $1268 \times 1024$ resolutions. The imaging instrument's software was written for SVGA in $800 \times 600$ resolution. In order to ensure that the computer is set up for this mode of operation the following statement should be placed in the autoexec.bat file:

$$
\text { SET VGA_CARD }=\mathbf{0 x 1 0 2 , 8 0 0 , 6 0 0}
$$

where the bolded portion is the interrupt vector, which varies for different video cards. A listing of the interrupts corresponding to the various video cards can be found in the Walk About manual ${ }^{33}$.

The firmware in the analog output card, National Instruments AT-AO-10, is set up for bipolar mode of operation. This means that the DACs are initialized to a value of 2048 when the computer is first powered on. When operating in the unipolar mode, as is the case for the control voltages for the focusing supplies, this value needs to be set to zero. A program written in assembler called inibrd.exe needs to be executed from within the autoexec.bat file to initialize the first six outputs of the analog output board to zero. Otherwise, when the signal conditioning circuit and the power supplies are turned on the supplies will have half of their full scale control signals applied to them.

The controller board, National Instruments GPIB-PCII, for the GPIB interface to the electrometer requires a device driver to be loaded allowing the software to communicate with the controller board. The following statement needs to be placed in the config.sys file:

\section{DEVICE = C:IGPIB-PCIGPIB.COM}

The path may be different depending on where the gpib.com file resides.

When GUIDE applications are first executed certain files will be created and are located in the directory files.gui. In order to ensure that the interface software functions properly, do not delete these files. The imaging instrument has files with the extension .img which contain calibration and setup 
information that is necessary when the program is executed. Data files containing the focusing voltages recorded during operation of the instrument are located in a directory called image.

\section{Hardware and Software Calibration}

Open loop control is used in the imaging instrument and no sampling of the actual focusing voltages is performed by the computer. Therefore, the software and hardware need to be calibrated so that the voliages displayed on the interface screen are approximately equal to the actual voltages being applied to the ion optics. It is not critical that these voltages be exactly the same since the overall performance is judged by the quality of the images. However, these voltages should be repeatable, allowing the images to be reproduced, at least close enough so that the focusing needs just a slight amount of tweaking. The calibration procedure requires that the power supplies be exercised through there entire operating range. In order to prevent any arcing, the outputs of the power supplies should be disconnected from the lens elements. After disconnecting the power supplies, the jumper, $\mathrm{J} 1$, on the signal conditioning circuit needs to be placed in the calibration position, as shown in Figure 31 . After the jumper has been placed in the calibration position, the software and hardware can be calibrated. At the DOS prompt type "image" and hit return. After a few seconds, the display should show the data interface. Click the mouse on the Calibrate button at the top of the screen. The screen should change and show the setup screen(see Figure 32). From within this screen the following changes can be performed:

1) The full voltage range that each of the power supplies is capable of being adjusted can be set. This is how the software and the hardware are calibrated.

2) The maximum deflector voltage can be adjusted, anywhere from 0 500 volts.

3) Also the calibration offsets for the deflector voltages can be set. These offsets are used to zero the deflector supplies.

4) The particular DAC channel for each power supply can also be selected. However, this should only be done if a hardware change has been implemented.

\section{Power supply calibration}

It is necessary to calibrate the power supplies with the software for several reasons: 1) if the instrument is being operated for the first time; 2) after any repair work is done to the signal conditioning circuit; 3) if a new power supply is installed on the instrument; and 4) if any large discrepancies between the voltages displayed by the software and the panels on the power supplies develops. The power supplies are calibrated by first adjusting the appropriate slider control to the designated position and then adjusting the corresponding potentiometer(s) to give the desired ranges of voltages for the given power supply. 


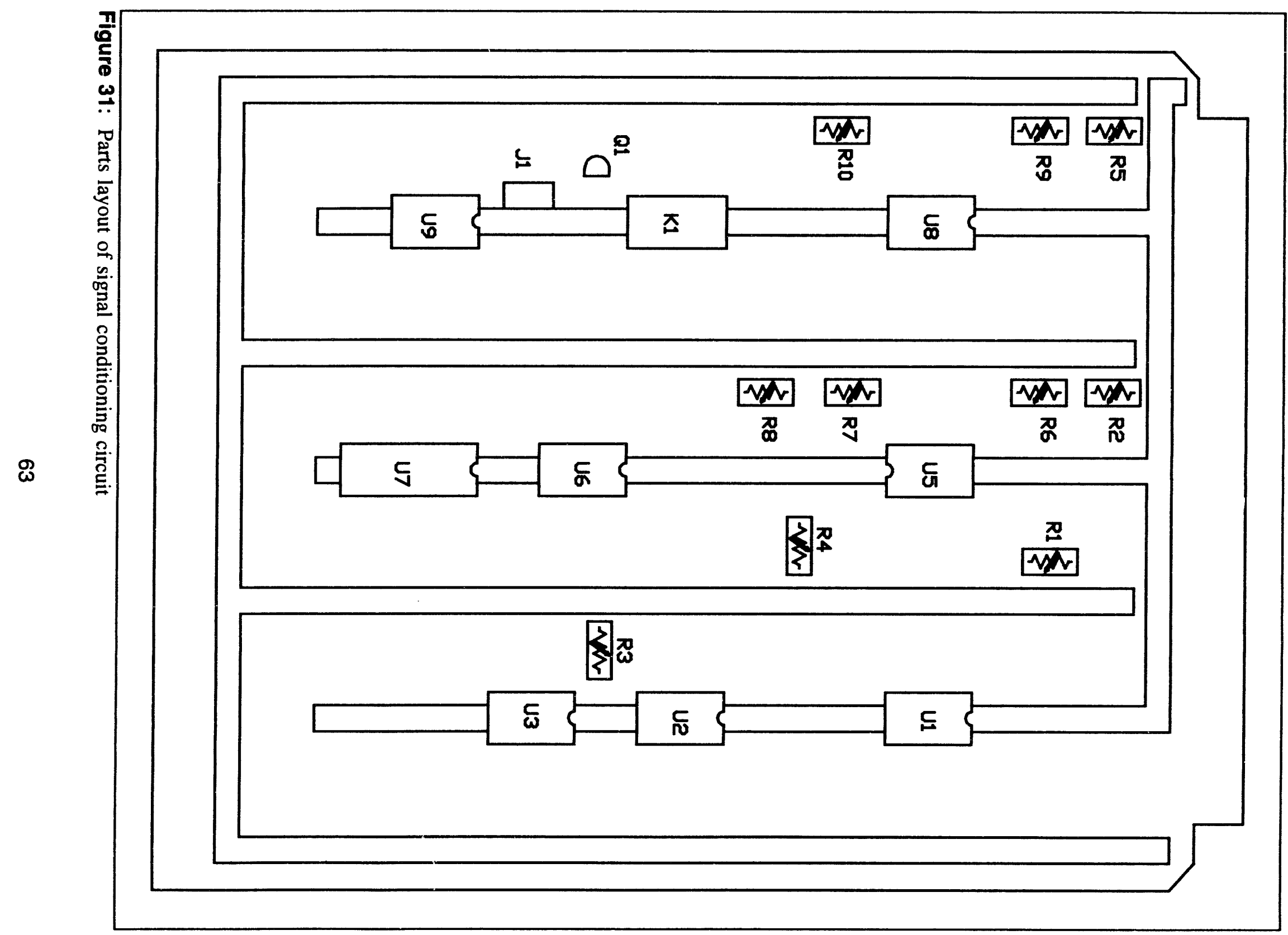




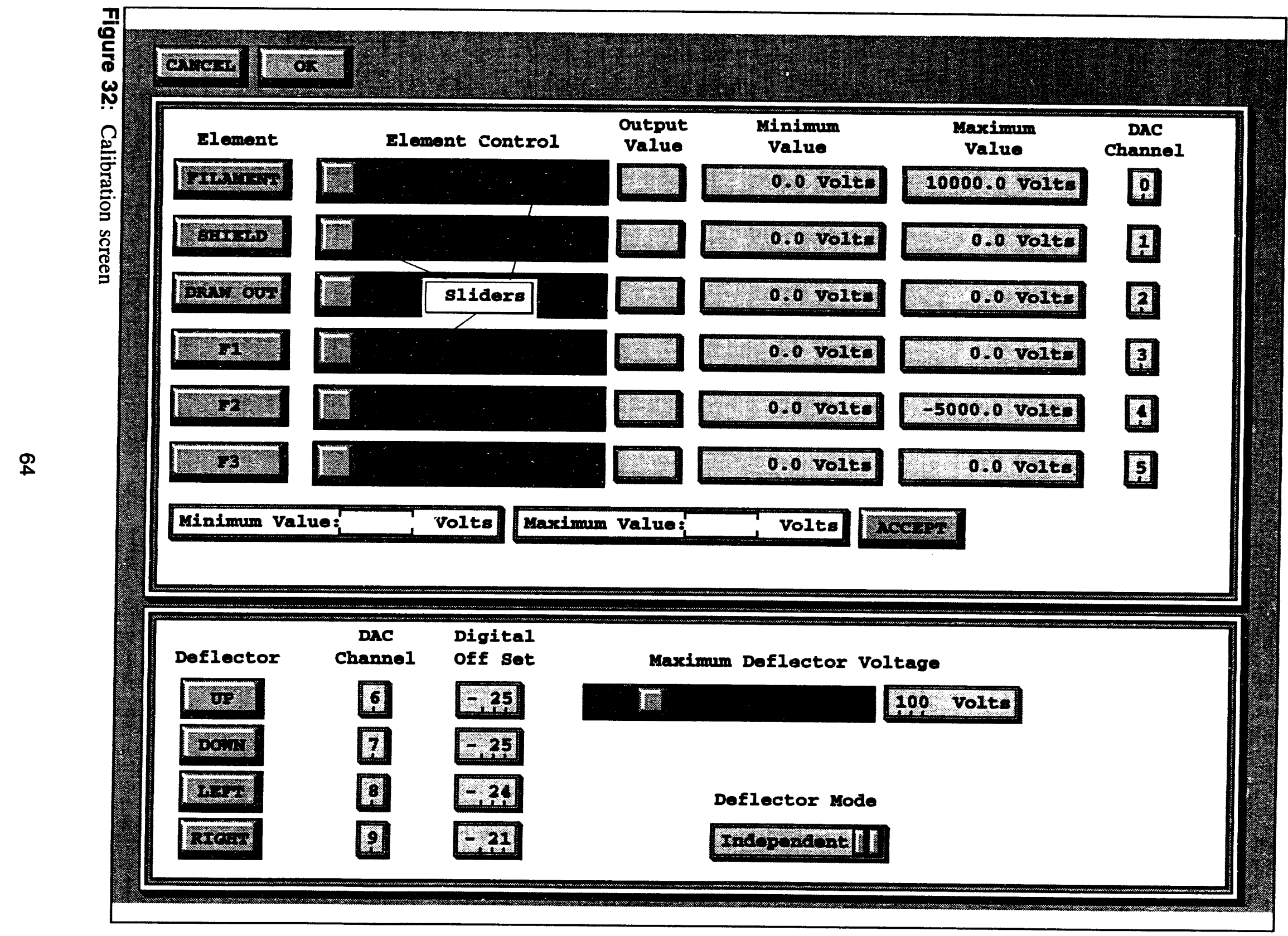


Master power supply. The master power supply has two potentiometer controls located on the signal conditioning circuit. The first control, $\mathrm{R} 1$, is used to zero the filament supply. Adjust the filament slider so the panel reads zero volts. Then adjust $\mathrm{R} 1$ so the power supply reads zero volts. Next, adjust the slider so that it is at the maximum value, all the way to the right. The maximum value of the filament supply is then adjusted using R2. Since R1 and R2 are on a summer circuit, there is a slight interaction between the settings on the two potentiometers. The crosscoupling between the two is due to the fact that the span is actually accomplished via the feedback resistor. Since the feedback resistor also controls the gain of the zeroing voltage, when the span is adjusted so is the gain for the zeroing voltage. In the original design, the circuit did not have the zeroing circuit. When the zero circuit was added this was the easiest place for it to be added. Unfortunately, the coupling between the two was a result of this placement. Therefore, it is an iterative approach to getting the zero and span adjustments correct. Alternate between zero and maximum until you are satisfied that the supply is calibrated.

After the master supply has been adjusted for zero and span, then enter the minimum and maximum values of the ion source voltage. These values will be used by the software for display purposes.

Zero and span - shield, draw out, $\mathbf{f 1}$ and f3. After the master supply has been calibrated, the supplies that track the master supply will have to be zeroed. The potentiometers R4 and R5 control the zero and span respectively. Bear in mind that this is the span of the tracking control and not the span for the individual control for each supply. Adjust the slider controls for each of the power supplies to zero volts. Then adjust potentiometer R4 so that the power supplies corresponding to the shield, draw out, F1 and F3 read approximately zero volts. Next, the span of the tracking control can be adjusted. Adjust the slider control on the computer screen for the master supply to maximum, while leaving the other slider controls at zero. Then adjust R5 until the outputs of the other supplies approximately match the output of the filament supply. Again there is a crosscoupling between the zero and span adjustments. So, alternate back and forth between zero and maximum until a satisfactory result is achieved.

Individual span adjustments. After the tracking span for the shield, draw out, F1 and F3 have been adjusted, then the individual span for each supply can be adjusted. The following list indicates which potentiometer effects which supply.

\author{
R6-Gain for F1 Span \\ R8-Gain for F3 Span \\ R9-Gain for Shield Span \\ R10-Gain for Draw Out Span
}

The procedure for adjusting these spans is virtually identical, so a generic procedure will be described.

First adjust the master supply to a point that is greater than the maximum delta volts for the particular supply. At this point the four tracking supplies should be at the same level as the master supply. Now adjust the slider corresponding to the power supply to the maximum level, completely to the right. By adjusting the potentiometer for that supply the span can be set to the particular range desired. Repeat this procedure for all four of the tracking supplies. 
After the span adjustments have been made, the minimum value should be entered. The maximum value is the value of the filament supply so this need not be entered. The software determines the maximum voltage offset from the filament each supply has and uses this value in determining the voltage value to display as the interface is being used.

F2 range adjust. The voltage on the second to last focusing element, F2, is not critical enough to require that it track the master supply. In fact, the range that $F 2$ is set varies from $+5 \mathrm{kV}$ to $-5 \mathrm{kV}$ and is independent of the polarity of ions being imaged. This is because this lens element is a substantial distance from the other elements. The control voltage for the Bertan supply is a $0-(-5)$ volt signal. The output from the computer is a 0 - 5 volt signal. Therefore, the signal is inverted in the signal conditioning circuit. The gain of the inverting amplifier is controlled by the potentiometer, R7. The output doesn't need to be zeroed. So, the only adjustment is the span of the control signal. Adjust the slider control for $F 2$ all the way to the maximum setting, fully to the right. Next adjust the potentiometer, $\mathrm{R} 7$, so that the output of the $\mathrm{F} 2$ supply is at $5 \mathrm{kV}$.

\section{Filament supply level detection}

At this point the power supplies should be calibrated with the software. The system could be operated at this point, however, the safety interlock would not be functional since the jumper, $\mathrm{J} 1$, is still in the calibrate position. In order to activate the interlock feature, remove the jumper and place it in the normal position. This will activate the interlock feature but the level at which the one shot kicks in must be adjusted. First, turn potentiometer, R3, fully counterclockwise. Then, adjust the master supply, as seen on the display, to the desired level at which you want the system to turn on. Next, adjust potentiometer, R3, until the power supplies come on. Now in order for any of the power supplies to operate, with the exception of F2, the master supply must be adjusted above this threshold.

\section{Deflector calibration}

The deflectors need to be zeroed and also the maximum value that the deflectors are allowed to be programmed needs to be set. To zero the deflectors, select the proper display on the spectrum solutions power supply. Then adjust the amount of offset by clicking the mouse within the digital off set panel on the interface display. After all of the deflector supplies have been zeroed, the voltage limit on the deflectors should be set. The maximum deflector voltage is set by either using the slider control or the panel to the right of the slider. The maximum value only needs to be adjusted once since this is a common parameter for all of the deflectors.

The deflector mode refers to the method which the software generates the voltages for each of the deflector plates. When the deflector mode is set to independent, the voltages appearing on the plates will be independent of each other. Where as in the bipolar mode, the voltages on parallel plates will be equal and opposite in polarity. Finally in the unipolar mode one of the plates in each pair of parallel plates will be held at ground while the other deflector is adjusted above and below ground potential.

This completes the calibration procedure. To view a list of all the calibration parameters click the mouse on the OK button on top of the screen. A listing of the parameters will appear. If you are satisfied with the settings then click on the accept button and the information will be stored to a file and will automatically be recalled each time the imaging instrument is used. 


\section{REFERENCES}

1. J.E. Delmore, A.D. Appelhans, and D.A. Dahl, Review of Scientific Instruments, Vol. 61, No. 1, Jan. 1990, pp. 633-635.

2. Anthony D. Appelhans, David A. Dahl, and James E. Delmore, Analytical Chemistry, Vol. 62, 1990, pp. 1679-1686.

3. B.J. Waclawski and E.W. Müller, Journal of Applied Physics, Vol. 32, No. 8, August 1961, pp. 1472-1475.

4. Erwin W. Müller, Science, Vol. 149, No. 3684, August 6, 1965, pp. 591-601.

5. P.J. Turner et al., Journal of Scientific Instruments, Series 2, Vol. 2, 1969, pp. 731-733.

6. E.W. Müller, J.A. Panitz and S.B. McLane, The Review of Scientific Instruments, Vol. 39, No. 1, January 1968, pp. 83-86.

7. A.R. Waugh, Journal of Scientific Instruments, Vol. 11, No. 1, January 1978, pp. 49-52.

8. Benninghoven, Rudenaurer and Werner, Secondary lon Mass Spectrometry, John Wiley and Sons, 1987.

9. B.K. Furman and G.H. Morrison, Analytical Chemistry, Vol. 52, No. 14, December 1980, pp. 2305-2309.

10. R.W. Odem et al., Analytical Chemistry, Vol. 55, No. 3, March 1983, pp. 574-578.

11. H.D. Wizemann, R. Jeske and K.H. Gaukler, International Journal of Mass Spectrometry and Ion Processes, Vol. 85, 1988, pp. 81-90.

12. N.A. Thorne, A. Dubus, F. Degrève, Scanning Electron Microscopy, No. IV, 1986, pp. 12551265.

13. Helmut Liebl, Journal of Applied Physics, Vol. 38, No. 13, December 1967, pp. 5277 - 5283.

14. Helmut Liebl, Analytical Chemistry, Vol. 46, No. 1, January 1974, pp. 22A-30A.

15. J.A. McHugh, J.C. Sheffield, L.R. Hanrahan, D.S. Simons, Procedings of the 25th Annual Conference on Mass Spectrometry and Allied Topics, Washington, 1977, pp. 706-708.

16. Basic vacuum Maintenance Training Workbook, Varian Associates Inc. Vacuum Products Division 1984.

17. D.J. Hucknall and D.G.Goetz, Vacuum, Vol. 8/9, pp. 615-620, 1987.

18. M. Audi and M de Simon, Vacuum, Vol. 37, No. 8/9, pp. 629-636, 1987. 
19. J.E. Delmore, A.D. Appelhans and E.S. Peterson, International Journal of Mass Spectrometry and Ion Processes, Vol. 108, 1991, pp.179-187.

20. Y. Satoh, M. Takebe, and K.linuma, Review of Scientific Instruments, Vol. 58, No. 1, January 1987, pp. 138-140.

21. Beck, Scott T., "Instrumental Design for Measurement of Surface Charge on Insulating Materials", thesis presented to Idaho State University, in 1991, in partial fulfillment of the requirements for the degree of Master of Science in Measurement and Control Engineering.

22. Galileo Electro-Optics Corp., Sturbridge, Ma 01518.

23. Joseph Ladislas Wiza, Nuclear Instruments and Methods, Vol. 162, 1979, pp. 587-601.

24. Galileo data sheet no. 9000, "Microchannel Plates", Galileo Electro Optics Corporation.

25. J.E. Delmore, A.D. Appelhans, International Journal of Mass Spectrometry and lon Processes, Vol. 68, 1986, pp 327-336.

26. D.A. Dahl, J.E. Delmore, and A.D. Appelhans, Review of Scientific Instruments, Vol. 61, No. 1, Jan. 1990, pp. 607-609. Program available from Idaho National Engineering Laboratory, EG\&G Idaho Inc., P.O. Box 1625, Idaho Falls, Idaho, 83415.

27. Kimbal Physics Corp., Wilton, NH 03086, eV Parts.

28. Installation, operation and Maintenance manual for model 5000 OEM..., Low Light Level Television Cameras, Cohu Inc. Electronics Division.

29. Newport Catalog, Newport corporation P.O. Box 8020, 18235 Mt. Baldy Circle Fountain Valley, Ca. 1989.

30. D.A. Dahl, Idaho National Engineering Laboratory, EG\&G Idaho Inc., P.O. Box 1625, Idaho Falls, Idaho, 83415.

31. NI-488 MS-DOS Software Reference Manual, National Instruments Corporation, April 1990

32. Keithley Model 617 Programmable Electrometer Instruction Manual, Keithley Instruments Division, Keithley Instruments, Inc. 28775 Aurora Road, Cleveland, Ohio 44139, August 1988.

33. David A. Dahl, Walk About Manual, Idaho National Engineering Laboratory, EG\&G Idaho Inc., P.O. Box 1625, Idaho Falls, Idaho, 83415. 
11
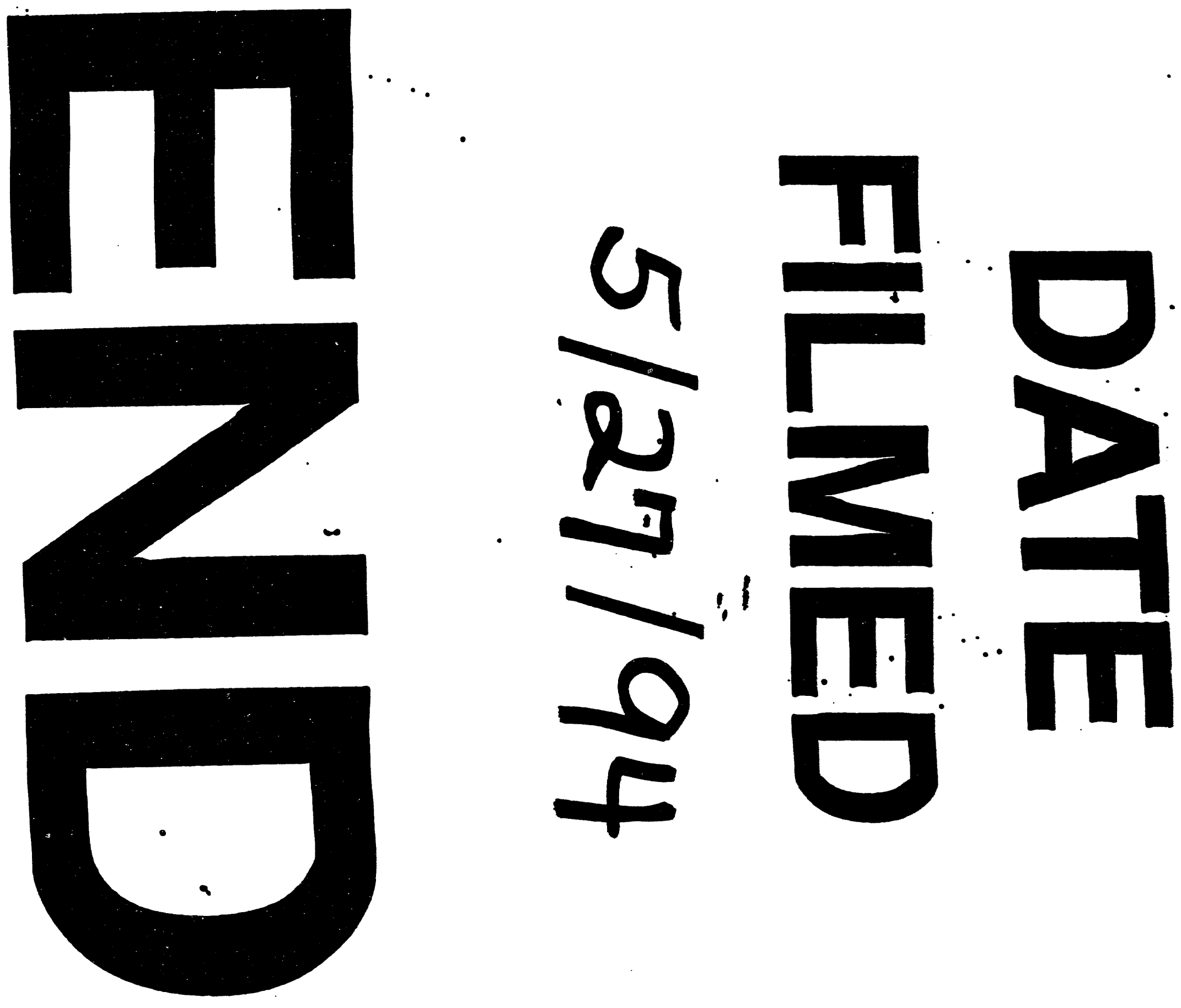
